\title{
ANÁLISIS DINÁMICO DE PUENTES ARCO DE HORMIGÓN PARA EL FERROCARRIL DE ALTA VELOCIDAD. CASO VIADUCTO SOBRE EL RÍO TAJO EN EL EMBALSE DE ALCÁNTARA
}

TRABAjo Fin DE MÁster

\author{
Por \\ María Elena Rodríguez Mesa \\ Ingeniero de Caminos, Canales y Puertos
}

Director

Alejandro E. Martínez Castro

Departamento de Mecánica de Estructuras e Ingeniería Hidráulica , Universidad de Granada

ETS de Ingenieros de Caminos, Canales y Puertos, C/ Severo Ochoa s/n, CP 18002

Granada (España)

Septiembre de 2016 

(C) Copyright 2016

por
María Elena Rodríguez Mesa 



\section{Resumen}

En la ingeniería de puentes de ferrocarril de alta velocidad, la tipología estructural de puentes arco de hormigón supone una elección idónea para el caso de luces medias o altas. No obstante, la ejecución de puentes bajo esta tipología es aún reducida a nivel nacional, así como el numero de publicaciones respecto a la respuesta dinámica ante el paso de cargas móviles en este tipo de estructuras.

La finalidad de este proyecto es analizar el Viaducto sobre el río Tajo en el embalse de Alcántara perteneciente a la línea de Alta Velocidad Madrid-Extremadura bajo el paso de cargas móviles y analizar su respuesta dinámica. Tras ello, realizar un informe técnico detectando los posibles efectos resonantes que puedan darse.

La solución al problema teórico de paso de cargas móviles se ha resuelto mediante una solución semianalítica. La parte espacial (modos y frecuencias) se resuelve mediante SAP 2000; la parte temporal se integra de forma exacta, evitando así los errores asociados a los métodos paso a paso.

Los resultados obtenidos muestran los problemas dinámicos específicos de la tipología de puentes arco de hormigón para su uso en el ferrocarril de alta velocidad. 



\section{Agradecimientos}

En primer lugar, me gustaría agradecer a mis padres, Ángel y María Ángeles, la oportunidad que me han brindado al poder estudiar esta carrera y posteriormente este Máster, no sólo por su ayuda económica, sino por el constante apoyo incondicional que he recibido por su parte. Mis padres que no tuvieron la oportunidad de estudiar, siempre me han apoyado en todas las decisiones de mi vida poniendo todo lo que estuviera a su alcance para ayudarme y darme lo mejor, cosa que no podré jamás agradecerles. Ellos me han enseñado a seguir luchando y a no rendirme y a que todo, si uno se lo propone se puede conseguir.

Por otro lado quisiera expresar mi agradecimiento al tutor de este trabajo, Alejandro Martínez Castro, por su dedicación, su compromiso y su disponibilidad en todo momento que le he necesitado. Sin sus numerosas lecciones sobre el tema y sin la plataforma para el cálculo semianalítico facilitada por él, este texto no podría haber sido posible.

Para finalizar, quiero recordar y agradecer a mis hermanos, amigos de Quéntar, compañeros de la carrera y del máster y a mi pareja, que han contribuído de manera especial al desarrollo de este documento y de mi vida universitaria y post-universitaria.

Comienzo una nueva etapa con mucha ilusión dejando atrás momentos negativos y positivos, los primeros me han hecho más fuerte y los segundos estarán siempre en mi corazón. 
Dedicado a mis padres, Ángel y María Ángeles y a Carlos, Luis y Juan Manuel. 


\section{Índice general}

$\begin{array}{ll}\text { Resumen } & \text { III }\end{array}$

Agradecimientos $\quad$ V

Capítulo 1. Introducción, objetivos y metodología 1

1.1. Introducción . . . . . . . . . . . . . . . . . . . . . . . 1

1.1.1. Motivación y problemática existente . . . . . . . . . . . . . 1

1.1.2. Particularidades de la tipología de puentes arco. . . . . . . . . . . . . . 2

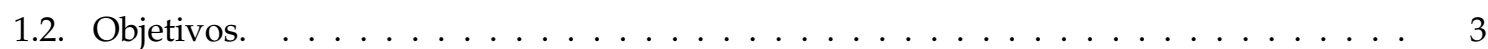

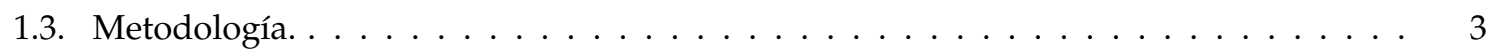

Capítulo 2. Revisión del estado de arte $\quad 5$

2.1. Tratamiento normativo del problema dinámico . . . . . . . . . . . . . . . 5

2.1.1. Introducción . . . . . . . . . . . . . . . . 5

2.1.2. Método disponibles para el cálculo dinámico y ámbito de aplicación . . . . . . 5

2.1.3. Método recomendado por la IAPF-2007 para el caso práctico. Limitaciones de la ins-

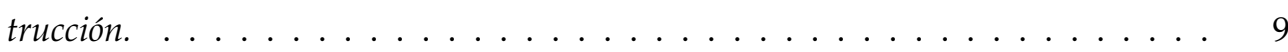

2.2. Solución al problema de cargas móviles . . . . . . . . . . . . . . . . . . . . . . 10

2.2.1. Trabajos y publicaciones sobre soluciones al problema de cargas móviles en puentes. . . 10

2.2.2. Métodos de integración en el dominio del tiempo. Planteamiento teórico. . . . . . . . . 11

2.2.3. Limitaciones de los métodos paso a paso. . . . . . . . . . . . . . . . . . . . . . . . 13

2.3. Puentes Arco para el Ferrocarril de Alta Velocidad. Caso del Viaducto sobre el Tajo. . . 13

Capítulo 3. Solución Semianalítica para el paso de carga móvil sobre la estructura 17

3.1. Introducción . . . . . . . . . . . . . . . . . . . . . . . . 17

3.2. Formulación del método . . . . . . . . . . . . . . . . . . . . . . 17

3.3. Generalización y ejecución del método . . . . . . . . . . . . . . . . . 26

3.4. Test de validación . . . . . . . . . . . . . . . . . . . . . . . . . 27

3.4.1. Planteamiento . . . . . . . . . . . . . . . 27

3.4.2. Test de validación 1: Viga de tres vanos . . . . . . . . . . . . . . . . . 28

3.5. Conclusión. Ventajas del método semianalítico . . . . . . . . . . . . . . . . . 34

Capítulo 4. Análisis dinámico del Viaducto sobre el Tajo 35

4.1. Introducción . . . . . . . . . . . . . . . . . . . . 35

4.2. Método de Cálculo . . . . . . . . . . . . . . . . . . . . . . . . 35

4.3. Modelo numérico de la estructura . . . . . . . . . . . . . . . . . . . . 36

4.4. Aciones. . . . . . . . . . . . . . . . . . . . . . 4 49

4.5. Resultados a obtener y comprobaciones a relaizar. . . . . . . . . . . . . . . . . 49

4.6. Resultados. . . . . . . . . . . . . . . . . . . . . . . 51 
4.6.1. Frecuencias propias y modos de vibración . . . . . . . . . . . . . . . . 51

4.6.2. Valores máximos y valores límite . . . . . . . . . . . . . . . . . . 65

4.6.3. Valores máximos. Tramo de arco. . . . . . . . . . . . . . . . . . . . 65

4.6.4. Valores máximos. Resto de estructura. . . . . . . . . . . . . . . . 66

4.6.5. Curvas envolventes de aceleraciones máximas . . . . . . . . . . . . . . 67

4.6.6. Curvas envolventes de desplazamientos máximos . . . . . . . . . . . . . 72

$\begin{array}{lll}\text { Capítulo 5. Conclusiones } & 77\end{array}$

$\begin{array}{ll}\text { Bibliografía } & 78\end{array}$ 


\section{Índice de cuadros}

2.1. Métodos de cálculo dinámico publicados en la instrucción IAPF-2007. . . . . . . . . 8

2.2. Valores de amortiguamiento $\zeta[\%]$ paar diversas tipologías de puentes en función de la luz $\mathrm{L}(\mathrm{m})$. Instrucción IAPF-2007 . . . . . . . . . . . . . . . . . . . . 9

3.1. Frecuencias de modos vibración, Test1. . . . . . . . . . . . . . . . . . . . . . . . . . . . . 29

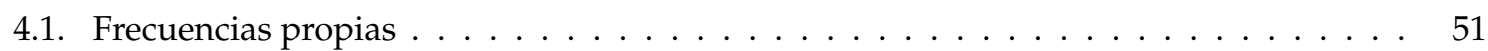

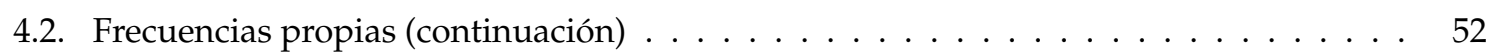

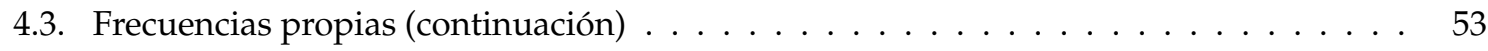

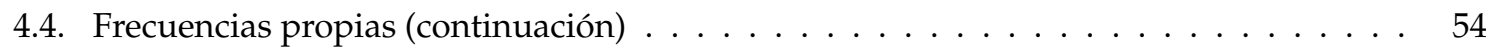

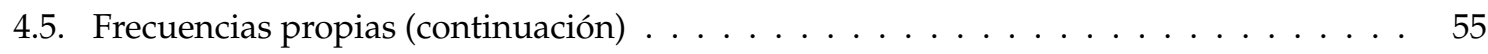

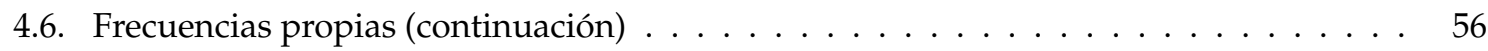





\section{Índice de figuras}

2.1. Alzado General. . . . . . . . . . . . . . . . . . . . . . . . . . . . . . . . . 14

2.2. Sección transversal del tablero . . . . . . . . . . . . . . . . . . . . . . . . 15

2.3. Alzado del arco . . . . . . . . . . . . . . . . . . . . . . . . . . . . 15

2.4. Planta del arco . . . . . . . . . . . . . . . . . . . . . . . . 15

3.1. Carga móvil sobre viga de sección variable. equilibrio de la rebanada . . . . . . . . . . 18

3.2. Discretización para un elemento barra y criterio de signos. . . . . . . . . . . . . . . 19

3.3. Esquema de línea de carga y puntos de postproceso en estructura. . . . . . . . . . . . . 27

3.4. Geometría de la viga, Test1. . . . . . . . . . . . . . . . . . . . . 28

3.5. Ejemplo de modo e vibración 11, Test1. . . . . . . . . . . . . . . . . . . . . . . . . . . 29

3.6. Aceleraciones en el punto central del primer vano por el métodod e Newmark. Test1. 30

3.7. Puntos de postproceso de la estructura para el método semianalítico y modo de vibra-

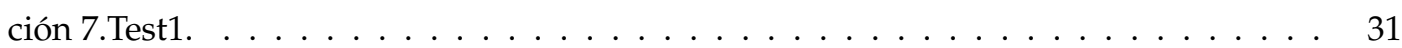

3.8. Aceleraciones en el punto central del primer vano por el método semianalítico.Test1. . 32

3.9. Comparación entre distintas metodologías de paso de cargas.Test1. . . . . . . . . . . . 33

4.1. Detalle de la malla empleada. Sección del estribo inicial . . . . . . . . . . . . . . 36

4.2. Detalle del modelo de las riostras. . . . . . . . . . . . . . . . . . . . . . . . . 37

4.3. Sección recta del modelo . . . . . . . . . . . . . . . . . . . . . . . 37

4.4. Modelo completo de la estructura . . . . . . . . . . . . . . . . . . 38

4.5. Modelo tipo viga del Arco . . . . . . . . . . . . . . . . . . . . . . 38

4.6. Alzado lateral con los distintos tipos de sección. Vanos 1-9 y 20-26 . . . . . . . . . 41

4.7. Alzado lateral con los distintos tipos de sección. Vanos 10 y 19 . . . . . . . . . . . . 42

4.8. Alzado lateral con los distintos tipos de sección. Vanos 11-13 y 16-18 . . . . . . . . . 43

4.9. Alzado lateral con los distintos tipos de sección. Vanos 14 y 15 . . . . . . . . . . . . . . 44

4.10. Vista en planta de la losa inferior. Situación y dirección de los apoyos de los primeros 11 vanos. . . . . . . . . . . . . . . . . . . . 46

4.11. Vista en planta de la losa inferior. Situación y dirección de los apoyos de los vanos 12-17 sobre el arco. . . . . . . . . . . . . . . . . . . . .

4.12. Vista en planta de la losa inferior. Situación y dirección de los apoyos de los vanos

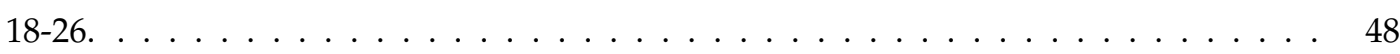

4.13. Puntos para cálculo de resultados. Vanos sobre el arco. . . . . . . . . . . . . . . . 50

4.14. Primer modo de vibración, de flexión acoplada de arco $(0.5638 \mathrm{~Hz})$. . . . . . . . . . 57

4.15. Segundo modo de vibración, de flexión acoplada de $\operatorname{arco}(0.68771 \mathrm{~Hz})$. . . . . . . . . . 58

4.16. Tercer modo de vibración, de flexión acoplada de arco $(1.1718 \mathrm{~Hz})$. . . . . . . . . . . 59

4.17. Modo de vibración $n^{\circ} 7(2.0154 \mathrm{~Hz})$. . . . . . . . . . . . . . . . . . . . . 60

4.18. Modo de vibración $\mathrm{n}^{\mathrm{o}} 46(6.1468 \mathrm{~Hz})$. . . . . . . . . . . . . . . . . . . . . 61

4.19. Modo de vibración $n^{0} 72$, torsión en vanos sobre el arco $(6.4461 \mathrm{~Hz})$. . . . . . . . . . 62

4.20. Modo de vibración $n^{0} 75$, torsión en vanos sobre el arco $(6.4547 \mathrm{~Hz})$. . . . . . . . . . 63

4.21. Modo de vibración $n^{\circ} 208(8.2072$ Hz) . . . . . . . . . . . . . . . . . . . . . . . . . . 64 
4.22. Envolventes de aceleración en puntos del vano 12 (sobre el arco). . . . . . . . . . . . 67

4.23. Envolventes de aceleración en puntos del vano 13 (sobre el arco). . . . . . . . . . . . 67

4.24. Envolventes de aceleración en puntos del vano 14 (sobre el arco). . . . . . . . . . . . 68

4.25. Envolventes de aceleración en puntos del vano 15 (sobre el arco). . . . . . . . . . . . 68

4.26. Envolventes de aceleración en puntos del vano 16 (sobre el arco). . . . . . . . . . . . . 69

4.27. Envolventes de aceleración en puntos del vano 17 (sobre el arco). . . . . . . . . . . . 69

4.28. Envolventes de aceleración en puntos exteriores al arco (vanos 1-10). . . . . . . . . . 70

4.29. Envolventes de aceleración en puntos exteriores al arco (vanos 11,18-26). . . . . . . . 70

4.30. Envolventes de aceleración en el punto 33 (vano 15) . . . . . . . . . . . . . . . . . . 71

4.31. Envolventes de aceleración en el punto 91 (centro del vano 25) . . . . . . . . . . . . . 71

4.32. Envolventes de desplazamiento en puntos del vano 12 (sobre el arco). . . . . . . . . 72

4.33. Envolventes de desplazamiento en puntos del vano 13 (sobre el arco). . . . . . . . . . 72

4.34. Envolventes de desplazamiento en puntos del vano 14 (sobre el arco). . . . . . . . . . 73

4.35. Envolventes de desplazamiento en puntos del vano 15 (sobre el arco). . . . . . . . . . 73

4.36. Envolventes de desplazamiento en puntos del vano 16 (sobre el arco). . . . . . . . . . 74

4.37. Envolventes de desplazamiento en puntos del vano 17 (sobre el arco). . . . . . . . . . 74

4.38. Envolventes de desplazamiento en el punto 64 (primer cuarto del vano 15, sobre el arco) 75 


\section{CAPÍtulo 1}

\section{Introducción, objetivos y metodología}

\subsection{Introducción}

\subsubsection{Motivación y problemática existente}

La alta velocidad es uno de los campos de la ingeniería de mayor interés en Europa. Su entrada se produjo en el año 1981 con la primera línea de TGV francés, aunque en España se produjo años después, el 21 de abril de 1992, con la salida del primer AVE desde la estación de Atocha de Madrid a Sevilla. España cuenta actualmente con más de $2600 \mathrm{Km}$ de líneas de alta velocidad en servicio, siendo la primera de Europa y segunda del mundo en número de kilómetros con altas prestaciones

Gracias a la alta velocidad, el tren vuelve a ser protagonista del transporte, ofreciendo un nivel de confort superior incluso a los del avión.Además, ha cambiado el concepto de largo recorrido ya que se reduce significativamente el tiempo de transporte para viajeros y mercancías.

Esta apuesta por el ferrocarril ha quedado patente en los últimos planes de infraestructuras entre los que destacan.

- Plan de infraestructuras 2000-2007: es el primer plan que apuesta por una mayor inversión en ferrocarril, como transporte de futuro, frente a la carretera con la ejecución de importantes obras como los $560 \mathrm{Km}$ hasta Lérida, de la línea Madrid-Barcelona-frontera francesa y otras obras importantes como las líneas Córdoba-Málaga o Madrid-Valladolid.

- El Plan Estratégico de Infraestructuras del sector del Transporte 2005-2010: aunque sustituye al PIT 2000-2007 impulsado por el gobierno anterior, sigue apostando por la alta velocidad, destinando un 42,82 \% de su inversión global (241.392 millones) a actuaciones en ferrocarril.

- Plan de Infraestructuras, Transporte y Vivienda 2012-2024: sigue otorgando prioridad al modo ferroviario, al fomento del transporte ferroviario de mercancías y a la culminación de los grandes ejes ferroviarios de alta velocidad. El transporte ferroviario acumula el $44 \%$ (61.302 millones) de los recursos inversores del horizonte temporal del PITVI siguiendo la tendencia de los últimos años.

Entre otras variables importantes para el diseño de un puente de ferrocarril destacan los efectos dinámicos, derivados de la alta velocidad. Estos efectos dinámicos producen un incremento, respecto a la respuesta estática, de las deformaciones y esfuerzos soportados por la estructura, pudiendo generar desplazamientos o aceleraciones nocivas para la estructura o comprometiendo la seguridad de circulación o el confort del viajero. Esto ocasiona que el análisis dinámico sea un gran condicionante del diseño en puentes de ferrocarril y que sea necesario un análisis dinámico exhaustivo, de ahí la motivación de este documento.

Con la aprobación de la Instrucción española de acciones a considerar en puentes de ferrocarril IAPF-07 [1], se establecen limitaciones dinámicas muy fuertes a cumplir en aceleraciones verticales, alabeos, flechas y giros máximos en el tablero de puentes de vía con balasto. 
Además de unas cada vez mayores exigencias para este tipo de estructuras, se suma como dificultad que los métodos clásicos de de resolución del problema de cargas móviles en el cálculo dinámico empiezan a estar obsoletos. Los métodos paso a paso han sido los más utilizados hasta el día de hoy, entre los que podemos destacar el método de Newmark-Beta.

Los problemas presentados por esta metodología se resumen en que los resultados obtenidos son aproximaciones en el dominio del tiempo, con lo cual aparecen errores inherentes a tal formulación. Por lo tanto, para obtener soluciones suficientemente aproximadas se necesitan se necesitan pasos de tiempo muy pequeños, ocasionando a su vez un gasto numérico inconcebible o simplificaciones que restan realidad a los resultados.

El método de cálculo semianalítico [2], autoría del director de éste Trabajo Final de Máster y ya empleado en informes de estructuras reales, no emplea una aproximación dominio temporal, lo que le permite realizar los barridos de trenes impuestos por la Instrucción de una manera precisa, además de disminuir abismalmente los tiempos de cálculo respecto a los métodos antecesores. El método semianalítico se convierte pues en un procedimiento ideal para el propósito de este proyecto , dar un enfoque práctico al cálculo dinámico de esta tipología concreta de puentes arco, de especial interés en este campo, desde un punto de vista ingenieril, puesto que permite la reducción del canto del tablero y puesto que, dada su gran esbeltez y bajo amortiguamiento, el problema dinámico será especialmente limitante en su diseño. Por otro lado, el estudio desarrollado pretende completar así el conocimiento respecto a esta tipología de puentes puesto que no existen demasiados estudios en profundidad al respecto en términos de respuesta dinámica al paso de las cargas impuestas por la Instrucción.

\subsubsection{Particularidades de la tipología de puentes arco.}

\section{Comportamiento estructural y realizaciones existentes}

La sección tipo cajón resulta óptima para hacer frente a los esfuerzos de flexión y torsión que solicitan a los tableros del puente, permitiendo la presencia de cargas excéntricas y con ello, de doble vía.

En cuanto al arco, es una estructura que resiste gracias a la forma que tiene. La geometría determina que los esfuerzos que lo solicitan sean casi exclusivamente de compresión, con la introducción de una componente inclinada en el terreno, por lo que son indicadas para cimentaciones resistentes. Sin embargo, bajo la sobrecarga de utlización, se producen tanto compresiones como flexiones.

En las estructuras conjuntas arco-tablero se dota al tablero de una rigidez suficiente para controlar las flexiones que producirán en el arco por la acción de la sobrecarga. El Viaducto sobre el Tajo en el embalse de Alcántara es un ejemplo de puente tipo Maillart en el que se disminuye la rigidez del arco aumentando la del tablero. Dicho de otro modo, el arco delgado es el antifunicular de la carga uniforme y es el tablero el encargado de resistir las flexiones que produce la sobrecarga.

En cuanto a las realizaciones llevadas a cabo según esta tipología estructural en el campo de la alta velocidad, a nivel nacional, son aún escasas. No obstante, entre ellas podemos destacar el Viaducto de Contreras, que salva el embalse del mismo nombre, constituye uno de los elementos más emblemáticos de las obras de construcción de la línea de alta velocidad Madrid-Valencia. 


\subsection{Objetivos.}

Los objetivos del presente proyecto se resumen en los siguientes puntos:

1. Desarrollo de una metodología que permita el análisis dinámico de puentes arco para el paso del ferrocarril de alta velocidad. Para ello, se utilizará el método semianalítico [2], autoría del director de este Trabajo Fin de Máster, tras verificar las ventajas de éste frente a los métodos tradcionales.

2. Conocimiento del tratamiento establecido por la normativa vigente (IAPF-2007 [1]) para el cálculo dinámico de estructuras de este tipo, estableciendo las variables necesarias para el análisis.

3. Cuantificación, con estas variables, de los problemas específicos de esta tipología en modelos 2D y 3D creados a este fin, destacando como inconvenientes:

a) La necesidad de una esbeltez estética para la estructura, que propicia modos acoplados de arco-tablero de baja frecuencia.

b) La aparición de modos de torsión acoplados a los efectos de flexión cuando un solo tren cruce la estructura, observables sólo en un modelo tridimensional.

4. Validación de una estructura existente a partir de un análisis dinámico completo, analizando qué variantes a introducir en el diseño o no influyendo sobre él, permiten una mayor aminoración de los efectos resonantes.

\subsection{Metodología.}

La metodología a seguir será la siguiente:

1. Revisión del estado del arte en lo relativo a soluciones para el paso de carga móvil en puentes arco de esta tipología.

2. Desarrollo del modelo de viga de Henchi en 2D con el objetivo de calibrar y demostrar las ventajas del método semianalítico frente a los métodos tradicionales.

3. Creación y validación del modelo tridimensional de la estructura mediante el software SAP2000, en el cual, se realiza el análisis modal.

4. Análisis dinámico exhaustivo de la estructura mediante el método semianalítico, estableciendo todas las variables que influyen o no sobre el diseño, permiten un mejor comportamiento dinámico. 



\section{CAPÍTUlO 2}

\section{Revisión del estado de arte}

\subsection{Tratamiento normativo del problema dinámico}

\subsubsection{Introducción}

El presente apartado se centra en la definición de los métodos actuales con los que se aborda el problema dinámico en base a la normativa actual española de acciones a considerar en puentes de ferrocarril, IAPF-2007 [1] :

Cualquiera que sea el método de evaluación de los efectos dinámicos, la envolvente de las solicitaciones dinámicas máximas obtenidas se caracterizarán mediante el coeficiente de impacto $\Phi$, encargado de mayorar las solicitaciones correspondientes al cálculo estático.

$$
\Phi=\frac{\max _{\text {din, real }}}{S_{\text {est }, \text { tipo }}}
$$

Donde:

- $\max S_{d i n, \text { real }}$ Solicitación dinámica máxima debida a todos los posibles trenes reales y velocidades de cirulación.

- $S_{\text {est,tipo: }}$ Solicitación estática debida al tren UIC71, definido en el apartado 2.3.1.1 de [1], situado en la posición más desfavorable.

\subsubsection{Método disponibles para el cálculo dinámico y ámbito de aplicación}

Método 1. Cálculo estático y coeficiente de impato envolvente:

Por este método, los esfuerzos dinámicos máximos producidos por los trenes reales, se obtendrán multiplicando los esfuerzos estáticos correspondientes al tren UIC71 (sistema del eurocódigo [3]), por un coeficiente de impacto envolvente (definido por las expresiones B.5 y B.6 de la instrucción [1], basada en los trenes de [4]).

El coeficiente de impacto $\Phi$ calculado por este método simplificado representa una envolvente de solicitaciones obtenida en un conjunto amplio de puentes y elementos estructurales reales, lo que linita su aplicación al ámbito que ha servido de base para su elaboración. En consecuencia, sólo será aplicable si cumplen todas las condiciones siguientes:

- Velocidades de circulación $\mathrm{v} \leq 220 \mathrm{Km} / \mathrm{h}$.

- Estructuras de tipología convencional definidas en los cuadros B.2 hasta B.5 de [1].

- Frecuencia propia de flexión del puente $f_{0}$ dentro de los límites de la figura B.1 de [1] 
En este ámbito de aplicación se admite que no se producen fenómenos resonantes y que las aceleraciones no superan los límites admisibles.

\section{Método 2. Coeficiente de impacto para trenes reales:}

Mientras que el método anterior proporcionaba un único coeficiente de impacto envolvente para todos los trenes y velocidades de circulación, por este método se obtienen los coeficientes de impacto de los trenes reales que sirvieron de base para calcular dicha envolvente, proporcionando así un mejor ajuste de la realidad.

Aplicable también para velocidades de circulación $\mathrm{v} \leq 220 \mathrm{Km} / \mathrm{h}$ y no siendo necesario que se cumplan los límites de frecuencias exigidos en el método anterior, podemos optar por el uso de una de estas dos opciones:

- Expresiones analíticas definidas en B.2 a B.5 de la Instrucción [1], sólo aplicable a las tipologías convencionales recogidas en los cuadros B.2 a B.5 de la misma. Se admite en este ámbito que no se producen fenómenos resonantes y que las aceleraciones no superan los límites admisibles.

- Obtención de los coeficientes de impacto mediante cáculo dinámico con trenes reales definidos en el apéndice C.3 [1], pudiéndose emplear para el cálculo alguno de los métodos que se describirán a continuación:

1. Cálculo dinámico mediante integración directa en el tiempo con cargas móviles.

2. Cálculo dinámico simplificado mediante impronta dinámica del tren.

3. Cálculo dinámico mediante integración directa en el tiempo con interacción vehículoestructura.

Método 3. Cálculo estático con coef. de impacto envolvente extendido a otras estructuras no resonantes:

Este método proporciona una envolvente de los trenes reales definidos en el apartado C.2 de la instrucción [1]. Es válido para cualquier rango de velocidades pero posee grandes restricciones en cuanto a masas, cocientes de velocidad y frecuencias y longitudes de vano por lo que será aplicable en pocos casos.

Si la estructura satisface los límites dados en el cuadro B.6 de tal instrucción, se podrá obviar el cáculo dinámico por un cáculo estático con el tren UIC71 afectado por su correspondiente coeficiente de impacto envolvente (primer método de aplicación). Esto incluirá los fenómenos resonantes normales que puedan afectar a este tipo de estructuras.

\section{Método 4. Cálculo dinámico mediante integración directa en el tiempo con cargas móviles:}

Este método se basa en la caracterización de los trenes como cargas móviles y la resolución en el tiempo del problema dinámico de la estructura, bajo dichas cargas. Es válido para cualquier rango de velocidades, no posee limitaciones de frecuencias ni de masas mínimas y puede aplicarse tanto a puentes isostáticos e hiperestáticos.

El proceso se basa en determinar las situaciones de carga más desfavorables (envolventes), simulando el paso de los trenes a las distintas velocidades de paso previsibles, desde la mínima de 
$20 \mathrm{Km} / \mathrm{h}$, hasta $1.2 \cdot V$, siendo $V$ la velocidad de proyecto de la vía. El incremento de velocidades entre cada paso será de un máximo de $10 \mathrm{Km} / \mathrm{h}$. Para dicho cálculo se utilizará el modelo HSLM (High Speed Load Model) definido en el Eurocódigo [3] y en el apéndice C.1 de la instrucción [3], para líneas interoperables. Finalmente, el coeficiente de impacto se determinará mediante la siguiente expresión:

$$
\Phi=\frac{\delta_{\text {din, }, \text { real }}^{\text {ideal }}}{S_{\text {est }, \text { tipo }}} \cdot\left(1+r \varphi^{\prime \prime}\right)
$$

Siendo:

- $\varphi^{\prime \prime}$, coeficiente que tiene en cuenta el efecto de las irregularidades de la via, definido por la expresión B.12 de las Instrucción, [1].

- r, coeficiente definido por la expresión B.12 de la Instrucción,[1].

Método 5. Cálculo dinámico simplificado mediante la impronta dinámica del tren:

Permite evaluar los efectos dinámicos sin realizar un cálculo dinámico con integración en el tiempo, resultando considerablemente más sencillo que la realización de un cálculo dinámico directo. La respuesta dinámica máxima se obtiene como producto de dos funciones que tienen expresión analítica, la impronta específica de cada tren y la línea de influencia dinámica del puente. Para ello existen dos métodos de cálculo, DER y LIR. Una documentación más detallada de éstos se pueden encontrar en los documentos del ERRI (Instituto Europeo de Investigación Ferroviaria) [5], o de forma más práctica en la referencia [6]. Aunque válido para cualquier rango de velocidad y sin limitaciones de frecuencias ni masas mínimas, este proceso tiene el inconveniente de que solo es aplicable a puentes isostáticos salvo casos especiales.

Método 6. Cálculo dinámico mediante integración directa en el tiempo con interacción vehículo estructura:

Los modelos de cargas móviles consideran que las cargas que transmiten las ruedas a los carriles son constantes en el tiempo y de valor igual a la carga nominal. En la realidad esta acción es variable debido, entre otros factores, a la suspensión de los vehículos. La consideración de los mecanismos de suspensión y la inercia de las masas suspendidas y no suspendidas conducen a los modelos con interacción entre vehículos y estructura. La utilización de éste métodopuede ser de utilidad para afinar el cálculo en alguna situación especial de proyecto, o bien como parte de un trabajo de investigación. Salvo casos excepcionales, estos modelos resultan de una complejidad excesiva para cáculos ordianrios de proyecto. Las solicitaciones obtenidas por este método suelen ser inferiores debidoa que parte de la energía permanece en los propios vehículos, siendo la diferencia más acusada en puentes isostáticos de luces cortas, con reducciones de más de un $30 \%$ de los efectos dinámicos máximos. En puentes hiperestáticos o situaciones no resonantes, sin embargo, los efectos de interacción, no suelen ser determinantes, por lo que puede prescindirse de estos.

Analizadas las características principales de cada método, se presenta el siguiente cuadro a modo de resumen: 


\begin{tabular}{|c|c|c|c|}
\hline lét & Telocidad & $\begin{array}{l}\text { ¿Cálculo dinámico? } \\
\text { ¿Efectos resonantes? }\end{array}$ & Otras Limitaciones \\
\hline $\begin{array}{l}\text { 1. Coeficiente de im- } \\
\text { pacto envolvente }\end{array}$ & $v \leq 220 \mathrm{Km} / \mathrm{h}$ & $\begin{array}{l}\text { No hay necesidad de } \\
\text { análisis dinámico. Un } \\
\text { coeficiente de impacto. } \\
\text { No se producen fenó- } \\
\text { menos resonantes ni es } \\
\text { necesaria la comproba- } \\
\text { ción de aceleraciones }\end{array}$ & $\begin{array}{l}\text { Puentes isostáticos } \\
\text { limitados a tipologías } \\
\text { convencionales reco- } \\
\text { gidas en la instrucción } \\
\text { [1]. Limitada la prime- } \\
\text { ra frecuencia propia } \\
\text { de flexión } f_{0}\end{array}$ \\
\hline $\begin{array}{l}\text { 2. Coeficiente de im- } \\
\text { pacto para los trenes } \\
\text { reales: 2.1.Mediante } \\
\text { expresiones analí- } \\
\text { ticas. 2.2.Mediante } \\
\text { cálculo dinámico con } \\
\text { trenesreales }\end{array}$ & $v \leq 220 \mathrm{Km} / \mathrm{h}$ & $\begin{array}{l}\text { No hay necesidad } \\
\text { de análisis dinámico. } \\
\text { Varios coeficientes } \\
\text { de impacto. No se } \\
\text { producen fenómenos } \\
\text { resonantes ni es nece- } \\
\text { saria la comprobación } \\
\text { de aceleraciones. 2.2. } \\
\text { Análisis dinámico con } \\
\text { trenes del apéndice C.3 } \\
\text { de la instrucción [1] }\end{array}$ & $\begin{array}{l}\text { 2.1.Puentes isostáticos } \\
\text { limitados a tipologías } \\
\text { convencionales reco- } \\
\text { gidas en la instrucción } \\
\text { [1]. 2.2.Puentes isos- } \\
\text { táticos sin limitación. } \\
\text { Empleo de los méto- } \\
\text { dos } 4,5 \text { o } 6 \text { para el } \\
\text { cálculo dinámico }\end{array}$ \\
\hline $\begin{array}{l}\text { 3. Cálculo estático con } \\
\text { coeficiente de impac- } \\
\text { to emvolvente exten- } \\
\text { dido a otras estructu- } \\
\text { ras no resontantes }\end{array}$ & $\begin{array}{l}\text { Cualquier rango } \\
\text { de velocidades }\end{array}$ & $\begin{array}{l}\text { Análisis dinámico con } \\
\text { trenes del apéndice C. } 2 \\
\text { de la instrucción [1]. } \\
\text { Puede obviarse el cálcu- } \\
\text { lo dinámico y aplicar } \\
\text { el método } 1 \text { si se satis- } \\
\text { facen condiciones. Tie- } \\
\text { ne en cuenta fenómenos } \\
\text { resonantes }\end{array}$ & $\begin{array}{l}\text { Aplicable en ciertas } \\
\text { estructuras con unos } \\
\text { rangos de masas, } \\
\text { cocientes de veloci- } \\
\text { dades y longitudes } \\
\text { de vano del puente } \\
\text { determinados }\end{array}$ \\
\hline $\begin{array}{l}\text { 4.Cálculo dinámico } \\
\text { mediante integración } \\
\text { directa en el tiempo } \\
\text { con cargas móviles }\end{array}$ & $\begin{array}{l}\text { Cualquier rango } \\
\text { de velocidades }\end{array}$ & $\begin{array}{l}\text { Análisis dinámico con } \\
\text { trenes del apéndice C.1 } \\
\text { de la instrucción [1].Ob- } \\
\text { jetivo de interoperabili- } \\
\text { dad. Tiene en cuenta fe- } \\
\text { nómenos resonantes }\end{array}$ & $\begin{array}{l}\text { No hay limitacio- } \\
\text { nes de freceuncias } \\
\text { mínimas. No hay } \\
\text { limitación respecto a } \\
\text { tipologías (válido para } \\
\text { puentes isotáticos e } \\
\text { hiperestáticos). }\end{array}$ \\
\hline $\begin{array}{l}\text { 5.Cálculo dinámico } \\
\text { simplificado me- } \\
\text { diante la impronta } \\
\text { dinámica del tren }\end{array}$ & $\begin{array}{l}\text { rango } \\
\text { dades }\end{array}$ & $\begin{array}{l}\text { El cálculo dinámico se } \\
\text { simplifica y no exige la } \\
\text { resolución en el tiempo, } \\
\text { utiliza fórmulas analíti- } \\
\text { cas. Tiene en cuenta fe- } \\
\text { nómenos resonantes }\end{array}$ & $\begin{array}{l}\text { No hay limitaciones } \\
\text { de frecuencias ni ma- } \\
\text { sas mínimas. Aplica- } \\
\text { ble sólo para puentes } \\
\text { isostáticos. }\end{array}$ \\
\hline $\begin{array}{l}\text { 6.Cálculo dinámico } \\
\text { simplificado me- } \\
\text { diante integración } \\
\text { directa en el tiem- } \\
\text { po con interacción } \\
\text { vehículo-estructura }\end{array}$ & $\begin{array}{l}\text { Cualquier rango } \\
\text { de velocidades }\end{array}$ & $\begin{array}{l}\text { Análisis dinámico con } \\
\text { trenes del apéndice C.1 } \\
\text { de la instrucción [1]. } \\
\text { Las cargas por eje de } \\
\text { los vehículos son tam- } \\
\text { bién variables. Tiene en } \\
\text { cuenta fenómenos reso- } \\
\text { nantes }\end{array}$ & $\begin{array}{l}\text { No hay limitaciones } \\
\text { de frecuencias ni ma- } \\
\text { sas mínimas. No hay } \\
\text { limitación respecto a } \\
\text { tipologías (válido para } \\
\text { puentes isostáticos e } \\
\text { hiperestáticos). Cálcu- } \\
\text { los más complejos y a } \\
\text { veces innecesarios. }\end{array}$ \\
\hline
\end{tabular}

Cuadro 2.1: Métodos de cálculo dinámico publicados en la instrucción IAPF-2007. 


\subsubsection{Método recomendado por la IAPF-2007 para el caso práctico. Limitaciones de la instrucción.}

Como se verá más adelante, el caso práctico consta de una estructura hiperestática de 26 vanos con 2 vanos de $45 \mathrm{~m}, 2$ vanos de $57 \mathrm{~m}, 6$ vanos de $54 \mathrm{~m}$ y 16 vanos de $60 \mathrm{~m}$.

Partiendo de la base de que la tipología de estudio no se encuentra recogida en las tablas B.2 a B.5 de la Instrucción [1] como tipología convencional así como el hecho de que la estructura del caso práctico es hiperestática, dan lugar a una reducción considerable del número de métodos que pueden utilizarse, quedando a disposición únicamente:

- Método 4: Cálculo dinámico mediante integración directa en el tiempo con cargas móviles.

- Método 6: Cálculo dinámico simplificado mediante integración directa en el tiempo con interacción vehículo-estructura.

Debido al grado de complejidad que los modelos de interacción vehículo-estructura introducen y al hecho de que en puentes hiperestáticos los efectos de interacción no sean determinantes en el cálculo, se descarta la utilización de este método aunque el ajuste de la respuesta sea ligeramente más afinado. Se opta, pues, por la utilización del método 4: Cálculo dinámico mediante integración directa en el tiempo con cargas móviles.

El caso de puentes de dos o más vías queda contemplado también en la instrucción [1], indicando que a partir de $v \geq 220 \mathrm{Km} / \mathrm{h}$ bastará considerar en el cálculo el paso de un tren por una única vía, que será la que resulte más desfavorable. esto permitirá en el caso práctico, la excitación de modos torsionales, motivación de este estudio.

Este documento pretende revelar elementos de la actual instrucción [1] que limitan los análisis dinámicos y que deben revisarse, evitando la validación dinámica de estructuras que en realidad no cumplen los límites. La instrucción exige en el cálculo dinámico un incremento de velocidades entre cada paso de un máximo de $10 \mathrm{Km} / \mathrm{h}$, valor obsoleto, debido principalmente al avance en precisión y tiempos de cálculo de las nuevas metodologías, como es el caso del método semianalítico [2]. El caso práctico del presente documento aplicará un incremento de velocidades entre cada paso de 1 $\mathrm{Km} / \mathrm{m}$ utilizando éste método, manifestando el hecho de que una diferencia de $10 \mathrm{Km} / \mathrm{h}$ puede obviar resultados importantes así como velocidades en las que puedan darse resonancias o máximos en aceleraciones, decantando la balanza de forma equivocada hacia un resultado favorable.

Otro tema a valorar, es el amortiguamiento tan bajo impuesto por la instrucción [1], debido a la falta de base de datos respecto a los puenets construidos en esta tipología. Así se pueden ver en la siguiente tabla:

\begin{tabular}{|c|c|c|}
\hline Tipo de puente & Luz $\mathrm{L}(\mathrm{m})$ & Limite inferior de amortiguamiento $\zeta[\%]$ \\
\hline \multirow{2}{*}{ Puentes de acero y mixtos } & $L<20$ & $0,5+0,125(20-L)$ \\
\cline { 2 - 3 } & $L \geq 20$ & 0,5 \\
\hline \multirow{2}{*}{ Puentes de hormigón estructural } & $L<20$ & $2,0+0,1(20-L)$ \\
\cline { 2 - 3 } & $L \geq 20$ & 2,0 \\
\hline
\end{tabular}

Cuadro 2.2: Valores de amortiguamiento $\zeta[\%]$ paar diversas tipologías de puentes en función de la luz L(m). Instrucción IAPF-2007 


\subsection{Solución al problema de cargas móviles}

\subsubsection{Trabajos y publicaciones sobre soluciones al problema de cargas móviles en puentes.}

El problema dinámico del paso de cargas móviles es de gran interés en el campo del diseño de puentes de ferrocarril y puentes carreteros. Dicho problema, consiste en la idealización del vehículo como un cojunto de cargas concentradas constantes que se desplazan sobe una línea de carga con velodidad constante. este análisis se pusode manifiesto tras los primeros problemas desestabilización de balasto ocurridos en algunas líneas europeas, que fueron estudiadas por Frýba[9] y Mancel [10], los cuales declararon la existencia del posible problema con las resonancias.

Con el tiempo, se ha tratado el problema dinámico a través de soluciones aproximadas pudiéndose obtener únicamente soluciones exactas para algunos casos concretos, como en los trabajos de Bleich[11] y Kryloff [12] para estructuras isostáticas (véanse también los trabajos mostrados en los libros de Frýba [3], Li y su [14] o Yang et al [15]). En el caso de estructuras hiperestáticas, el número de trabajos es inferior, pudiéndose destacar entre ellos los trabajos de Chen y Li [16] para cargas exponenciales muy concretas, el trabajo de Hayashikawa y Watanabe [17] que idealizan vigas de canto variable mediante vigas de canto constante a tramos o la aportación de henchi et al [18] que obtiene la solición en el dominio de la frecuencia.

Para una carga general sobre una estructura genérica, la técnica acude al métods de integración paso a paso, Step-by-Step", (véanse en el libro de Clogh y Penzien [19]), y en particular, como método más difundido, el método de integración Newmark-Beta [20].

De entre los numerosos estudios dinámicos, el artículo sobre el viaducto de Sesia de K.Liu et al.[8] merece mencionarse pues presenta un estudio en el que se aborda la interacción vehículo-estructura sobre puentes de alta velocidad mediante integración directa Newmark-Beta. Además, el artículo incluye mediciones in situ tras la construcción del viaducto y ha obtenido conclusiones interesantes respecto al amortiguamiento de este tipo de estructuras.

El problema de la metodología de integración directa es que al fin y al cabo, se trata de una aproximación en el dominio del tiempo mediante el parámetro del paso temporal, de modo que para obtener soluciones suficientemente aproximadas dad la baja tasa de amortiguamiento impuesta por la instrucción [1], se necesita de pasos de tiempo muy pequeños con el gasto numérico que eso conlleva.

Como solución a este problema aparecen diversas referencia como el artículo de Dugush y Eisenberg [27] en el que se propone una descripción de los modos de vibración mediante series polinómicas infinitas. De este modo, la parte espacial se resuelve de forma exacta mediante el método de la rigidez dinámica y la solución temporal es analítica correspondiendo así a una serie infinita.

Otro método es el denominado semianalítico y propuesto por Castro et al. [28],[2],[29] y [30], en la Universidad de Granada. esta metodología se basa en la resolución espacial mediante discretización por elementos finitos y una resolución analítica en el dominio del tiempo, por lo cual desaparecen todos los errores inherentes a la aproximación en el tiempo de los métodos paso a paso. Este método semianalítico, ha sido reconocido por la comunidad científica y aplicado con gran éxito en el diseño 
del puente de Santa Ana, puente arco de de la línea de Córdoba-Málaga [31].

\subsubsection{Métodos de integración en el dominio del tiempo. Planteamiento teórico.}

La solución al problema dinámico que se plantea en el problema de paso de cargas móviles se resuelve de forma numérica mediante dos metodologías:

- Métodos indirectos: Son los métodos que aplican el principio de superposición y por lo tanto sólo son aplicables para comportamiento lineal de la estructura. el más conocido es el método de superposición modal, el cual resuelve el problema mediante integración temporal de las ecuaciones modales, separando variable espacial y temporal. Requiere de métodos paso a paso (directos) para la resolución numérica de la integral temporal, de ahí que se utilicen directamente estos últimos por su mayor versatilidad.

- Métodos directos: Son los métodos más generales, pues permiten no linealidades en la estructura. Consisten en la integración directa de las ecuaciones de equilibrio para todos los grados de libertad de la estructura en cada instante. Entre los más ampliamente usados se enceuntran los métodos paso a paso de Newmark-Beta, Hughes, el método de Wilson y otros mucho más que se han ido añadiendo a la bibliografía (los principales métodos numéricos se encuentran más ampliamente desarrollados en las referencias [20],[32],[33] y [34]). Aunque requieren de un mayor esfuerzo de cálculo son más utilizados que los anteriores.

\section{Los métodos paso a paso}

Los métodos paso a paso tienen numerosas variantes, pero todos ellos se basan en definitiva en dividir la carga dinámica y la respuesta del sistema en una serie de "pasosz calcular la respuesta en cada "paso. ${ }^{a}$ partir de las condiciones iniciales al principio del mismo. La respuesta en cada "paso"se obtiene con un análisis independiente del resto de casos, asumiendo como aproximación que ene ese "paso. ${ }^{\text {el }}$ sistema se mantiene lineal aunque las propiedades en el conjunto del sistema van variando.

La ecuación de partida para un algoritmo de integración temporal de primer orden, o problema de Cauchy, viene dada por:

$$
\dot{y}(t)=f(t, y(t)), y\left(y_{0}\right)=y_{0} ; t \in\left[t_{0}, t_{f}\right]
$$

No obstante, para aproximar la dinámica de una estructura general se deberá acudir a algoritmos de integración temporal de segundo orden que se pueden expresar en forma compacta de la siguiente forma:

$$
M \cdot \ddot{w}=F(w, \ddot{w}, t)
$$

Donde el vector F y la matriz $\mathrm{M}$ son, en general, funciones no lineales de $(w, \dot{w}, t)$. Para poder trabajar métodos de primer orden, se puede reconvertir el sistema anterior a través del siguiente cambio: 


$$
y=\frac{w}{\dot{w}} \longrightarrow \dot{y}=\frac{\dot{w}}{M^{-1} \cdot F(w, \dot{w}, t)}=F(t, y(t))
$$

Un método numérico de integración temporal determina la solución al problema dinámico en un instante dado a partir de la información obtenida en una serie de instantes previos. De este modo, para cada instante $t_{n}$ obtenemos un valor de $y_{n}(\mathrm{n}=0,1, \ldots, \mathrm{N})$ a partir del conocimiento del valor de la misma en los $\mathrm{k}$ instantes anteriores:

$$
\sum_{j=0}^{k} \alpha_{j} y_{n+j}=h \cdot \Phi\left(y_{n+k}, y_{n+k-1}, y_{n+k-2}, \ldots, y_{n}, t_{n+k} ; h_{n+k}\right)
$$

En la ecuación 2.6 se recogen todos los métodos de integración posibles. Aunque se establecen numerosas variantes en función de los parámetros utilizados, como por ejemplo: "métodos de un paso y multipaso"si se obtiene la respuesta a partir de la información de un paso o de varios pasos anteriores o "métodos de paso fijo y de paso variable"si se utiliza un único o varios valores de paso de integración a lo largo de todo el proceso, los métodos paso a paso suelen clasificarse en dos grupos principales:

\section{- Métodos explícitos:}

Los métodos explícitos usan la ecuación diferencial en un tiempo $t_{n+1}$ para predecir una solución en $t_{n}+\Delta t_{n}$. La particularidad de este esquema es que la expresión 2.6 permite despejar $y_{n+k}$ conocidos los valores anteriores, $y_{n+j}, j=0,1, . ., k-1$.

Se conoce que todos los métodos explícitos son condicionalmente estables con respecto al tamaño del incremento de tiempo, de modo que para el cálculo de una estructura real, se requiee de muy pequeños incrementos de tiempo para obtener una solución estable.

Como ejemplos de un esquema de integración explícito, se pueden citar los métodos de RungeKutta explícitos cuya formulación es la siguiente:

$$
y_{n+1}=y_{n}+h_{n} \Phi_{n}\left(y_{n}, h_{n}\right) ; n=0,1, \ldots, N-1
$$

\section{- Métodos implícitos}

Los métodos implícitos satisfacen la ecuación diferencial en un tiempo $t_{n}$ después de que la solución en $t_{n}-\Delta t_{n}$ haya sido encontrada. A diferencia de los esquemas explícitos, ahora no es posible despejar $y_{n+k}$ de la ecuación 2.6 una vez que conocemos $y_{n+j}, j=0,1, \ldots, k-1$, de modo que se deberá resolver un sistema de ecuaciones lineales en cada paso. Sin embargo, en estos algoritmos es posible usar incrementos de integración mayores al anterior, pudiendo ser condicional o incondicionalmente estables. Para los métodos que son incondicionalmente estables la única limitación para el tamaño del incremento del tiempo es la precisión de la solución. 
Como ejemplo, citar el método de Adams implícito de un paso conocido como la regla de los trapecios y es de la forma:

$$
y_{n+1}-y_{n}=\frac{\Delta t_{n}}{2}\left(f\left(t_{n}, y_{n}\right)+f\left(t_{n+1}, y_{n+1}\right)\right)
$$

También se introduce en esta categoría el método más utilizado actualmente, el método de Newmark-Beta, desarrollado más extensamente en el apéndice A.

\subsubsection{Limitaciones de los métodos paso a paso.}

La metodología paso a paso se basa en una integración numérica de tipo incremental en el dominio del tiempo, condición que no es sino una aproximación de dicho dominio a través del parámetro de paso de tiempo. En estructuras reales, estos métodos se alejan de ser aplicables desde un punto de vista práctico pues para que la respuesta sea realista, se requiere de pasos de tiempo muy pequeños lo que conduce a costes numéricos desproporcionados o excesivas simplificaciones que restan validez a los modelos. La baja tasa de amortiguación impuesta por al instrucción [1] para las estructuras mixtas se suma como problema pues la solución requiere de pasos de tiempo más pequeños todavía en comparación con otras estructuras existentes.

Estos problemas no se dan con el método semianalítico pues elimina la aproximación en el dominio temporal y no da resultados en forma de series infinitas. Aunque sigue existiendo una aproximación numérica derivada del modelo espacial mediante elementos finitos, los resultados son los más fiables y rápidos de entre los métodos actuales posibles. Por ello en el presente documento, se ha optado por el empleo de esta metodología semianalítica en todos los cálculos llevados a cabo tras contrastar ésta con las metodologías clásicas de integración directa en dos ensayos numéricos presentados en el siguiente capítulo.

\subsection{Puentes Arco para el Ferrocarril de Alta Velocidad. Caso del Viaducto sobre el Tajo.}

La Línea de Alta Velocidad Madrid-Extremadura tiene una longitud total de $450 \mathrm{Km}$, con una inversión prevista de 2713 millones de euros para los tramos encomendados a Adif. El trazado se ha diseñado como doble vía electrificada en alta velocidad en ancho internacional (UIC) para velocidades máximas de $350 \mathrm{Km} / \mathrm{h}$ para tráfico mixto de trenes de viajeros y mercancías en todo el recorrido.

El tramo Talayuela-Cáceres tiene una longitud de $127.5 \mathrm{Km}$ y discurre íntegramente por la provincia de Cáceres.

Sin lugar a dudas, en este tramo destaca la construcción del viaducto para salvar el río Tajo, en una zona donde dicho río, debido a la influencia del embalse de Alcántara, presenta un ensanchamiento del cauce.

Para salvarlo, sin pilas intermedias sobre el río, atendiendo a los condicionantes expresados en la correspondiente Declaración de Impacto Ambiental, se ha resuelto mediante un gran arco de hormigón de $324 \mathrm{~m}$ de luz entre los apoyos situados fuera del embalse.

El viaducto tiene una longitud total de $1488 \mathrm{~m}$. La distribución de luces del viaducto viene influenciada por el salto del Río Tajo, fragmentándose el tablero sobre el arco en seis vanos de $54 \mathrm{~m}$. Lo 
vanos de acceso se plantean de $60 \mathrm{~m}$, intercalándose entre ellos dos vanos de transición, uno a cada lado del arranque del arco, de $57 \mathrm{~m}$. Esto da lugar a una distribución de luces armoniosa y adecuada de los 26 vanos, que es la siguiente: $45 m+9 \times 60 m+57 m+6 x 54+57 m+7 \times 60 m+45 m$.

El trazado del viaducto presenta una primera parte sobre una curva de gran amplitud y el resto del puente queda inscrito en una recta.

El elemento más emblemático de este viaducto es el arco de $324 \mathrm{~m}$ de luz entre apoyos, elevándose sobre sus cimentaciones una altura de $70 \mathrm{~m}$ (flecha del arco) y sobre el nivel máximo de embalse una altura superior a los $80 \mathrm{~m}$.

El arco está formado por una sección rectangular achaflanada hueca, tipo cajón de canto variable. $\mathrm{El}$ arranque del arco tiene un canto de $4 \mathrm{~m}$ y una anchura de $12 \mathrm{~m}$. En la clave, punto más alto del arco, la sección se reduce hasta un canto de 3,5 m y y $6 \mathrm{~m}$ de ancho.

El tablero presenta sección cajón de hormigón pretensado con canto constante de $3.6 \mathrm{~m}$ y anchura de $14 \mathrm{~m}$, permitiendo alojar la plataforma de vía doble de $10.10 \mathrm{~m}$ de ancho y dos paseos de 1,75 m a cada lado más otros elementos para el buen funcionamiento de la línea del ferrocarril.

El ancho inferior del cajón es de $5 \mathrm{~m}$ mientras que la parte superior presenta una anchura de 6.5 $\mathrm{m}$, y se dota de voladizos laterales que completan la anchura total de la sección hasta los $14 \mathrm{~m}$.

Las pilas tienen una altura variable para adaptarse al perfil del trazado, alcanzándose alturas superiores a los $70 \mathrm{~m}$ en la zona más cercana al cruce del río. Todas las pilas constan de un único fuste de sección cajón hueca excepto en su cabecero, donde la sección se maciza para recibir las cargas recibidas por el tablero.

Un aspecto a destacar para la construcción de este viaducto es la necesidad de empleo de hormigones de alta resistencia. Tanto para la ejecución del arco como para la parte del tablero situada sobre el mismo se ha previsto la utilización de un hormigón de $700 \mathrm{~kg} / \mathrm{cm}^{2}$ de resistencia característica.

En cuanto a las cimentaciones de arco, pilas de vanos de acceso y estribos, todas ellas se resuelven mediante grandes zapatas sobre el sustrato rocoso para repartir las considerables cargas que han de soportar.

En las figuras 2.1 y 2.2 se presentan el alzado general del viaducto y la sección transversal del tablero.

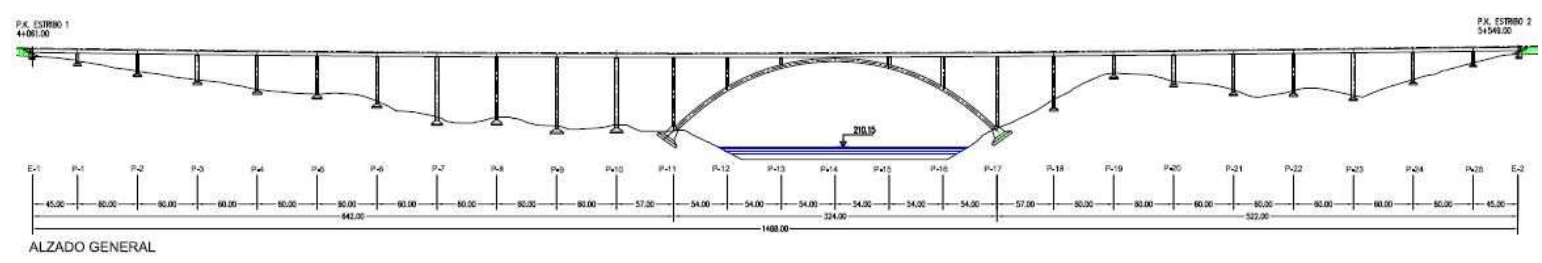

Figura 2.1: Alzado General. 


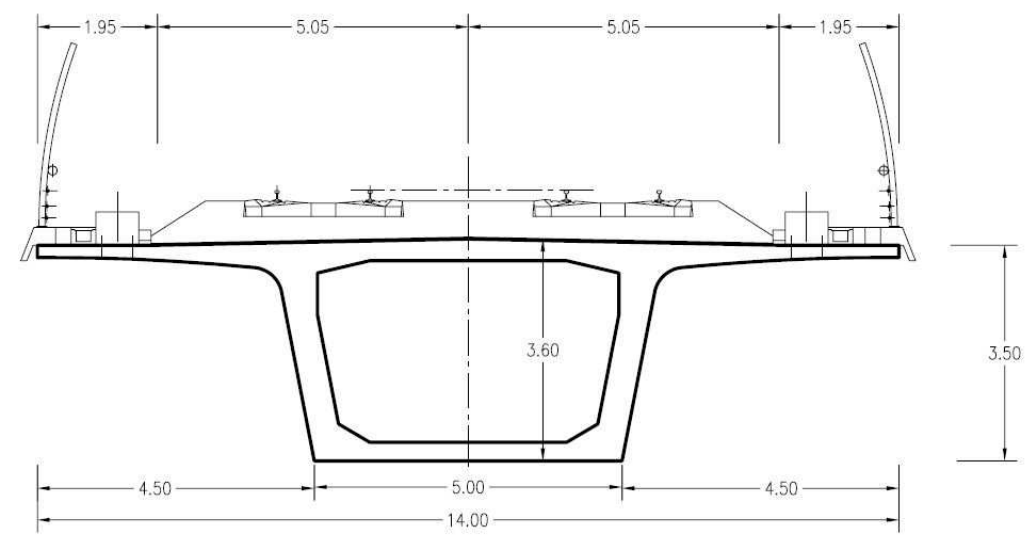

SECCION TRANSVERSAL DE TABLERO

Figura 2.2: Sección transversal del tablero

Las figuras 2.3 y 2.4 muestran el alzado y la planta longitudinal del arco respectivamente.

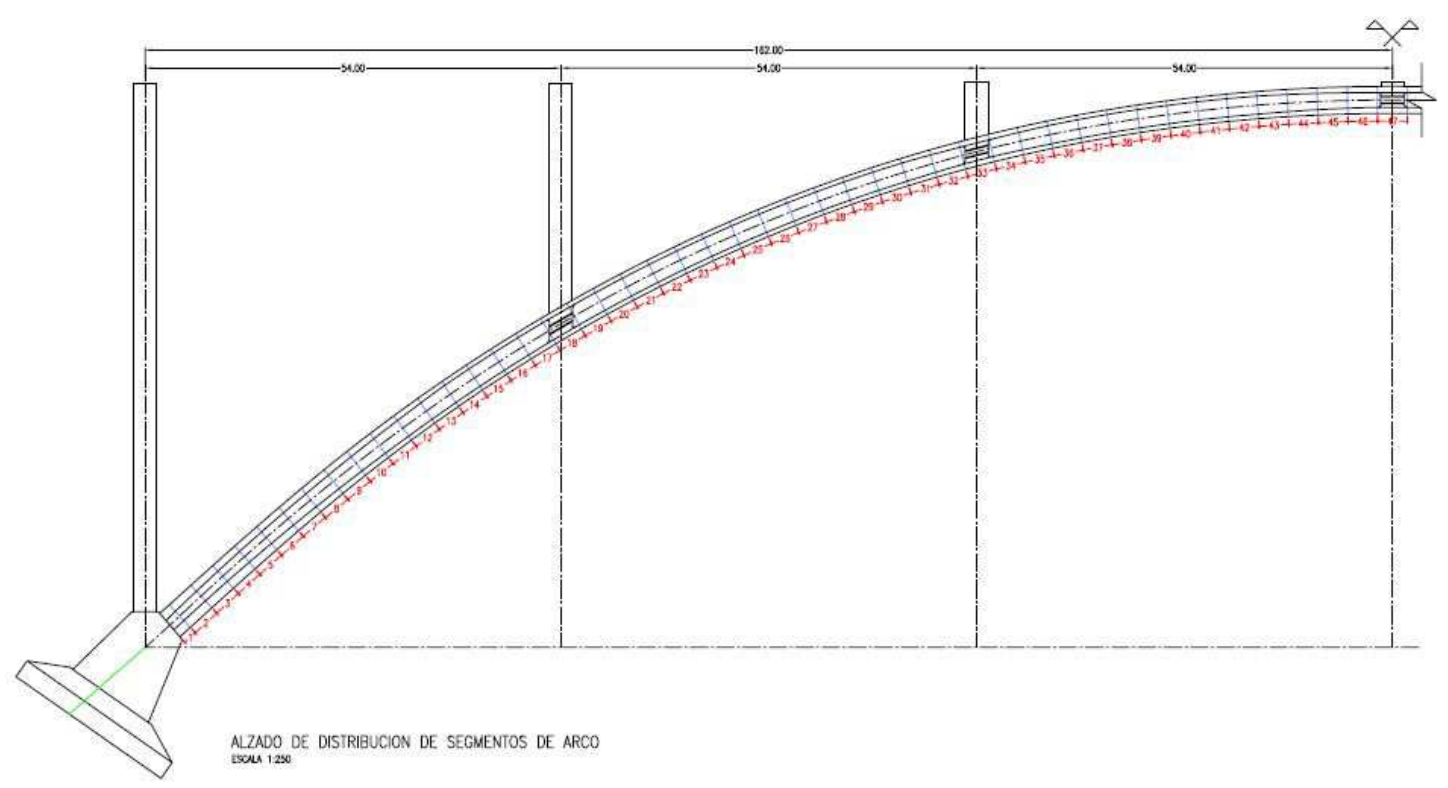

Figura 2.3: Alzado del arco

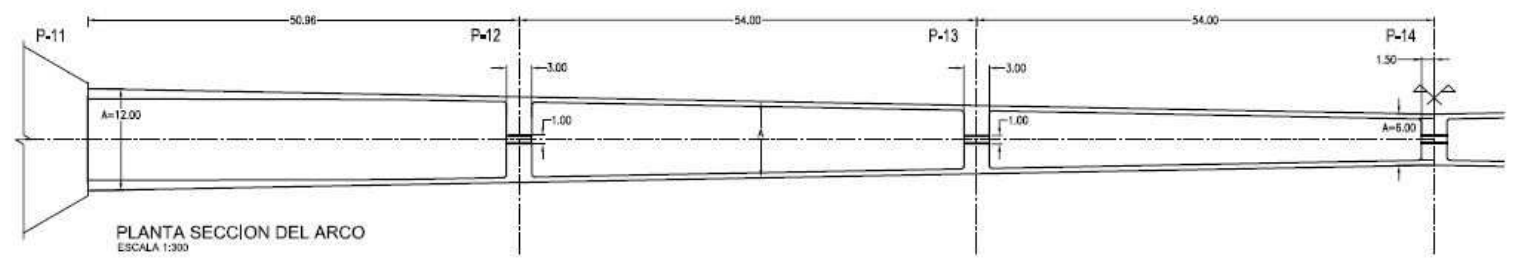

Figura 2.4: Planta del arco 



\section{CAPÍtulo 3}

\section{Solución Semianalítica para el paso de carga móvil sobre la estructura}

\subsection{Introducción}

El método semianalítico, desarrollado por A.Martínez-Castro, P.Museros y A.Castillo-Linares, (Universidad de Granada) y publicado en la revista Journal of Sound and Vibration[2], basándose en el modelo de viga de Euler-Bernouilli propone una solución al problema de paso de cargas móviles sobre vigas [28] basándose en la superposición modal con discretización de la parte espacial mediante elementos finitos y con soluciones analíticas exactas en la parte temporal. Este método posee una aproximación numérica, procedente de la discretización espacial mediante el modelo de Elementos finitos, no obstante, se eliminan importantes fuentes de error asociadas al paso de tiempo usado en los métodos de integración directa clásicos.

En lo que sigue, se desarrollará la formulación del método así como su aplicación a casos prácticos que, junto con un análisis de los métodos de aproximación paso a paso, nos permitirá comparar ambos métodos en aproximación y tiempo de cálculo. Como quedará patente, esta metodología aventaja en mucho a los métodos tradicionales de integración paso a paso, salvando los problemas de imprecisión y lentitud en el cálculo de estos últimos. En la actualidad, los métodos de integración paso a paso se emplean ampliamente en los proyectos de puentes. No obstante, ante la complejidad de cálculo numérico se supone su aplicación a las exigencias y bajas tasas de amortiaguamiento impuestos por la norma IAPF, deberán ser sustituídos irremediablemente por en un futuro próximo.

\subsection{Formulación del método}

Para la formulación del método nos vamos a basar en los siguientes pasos:

1. Obtención de la ecuación de gobierno de uan viga de canto variable.

2. Obtención de la formulación matricial de un elemento barra y generalización a toda la estructura.

3. Resolución del sistema.

4. Solución para cada modo de vibración, pues se ha desacoplado el sistema. Introducción de amortiguamiento en el problema.

Paso 1.Ecuación de Gobierno de una viga de sección variable en dinámica.

Se considera una viga de sección variable, sometida a una carga $q(x, t)$ representativa de una fuerza puntual $p_{0}$ que se desplaza por el eje $x$ de la viga a una velocidad constante $v$. La función $q$ se 'puede definir mediante una delta de Dirac según la ecuación 3.1: 


$$
q(x, t)=p_{0} \cdot \delta(x-v t)
$$

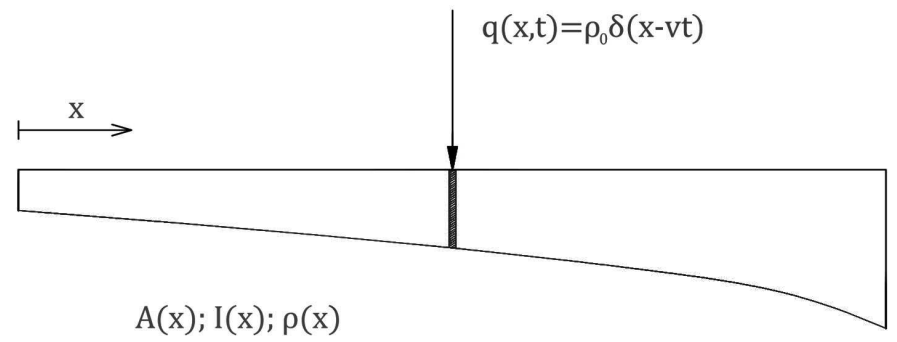

$\mathrm{A}(\mathrm{x}) ; \mathrm{I}(\mathrm{x}) ; \rho(\mathrm{x})$

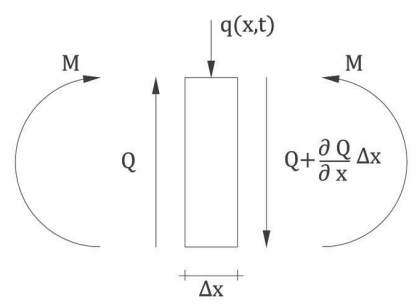

Equilibrio de fuerzas

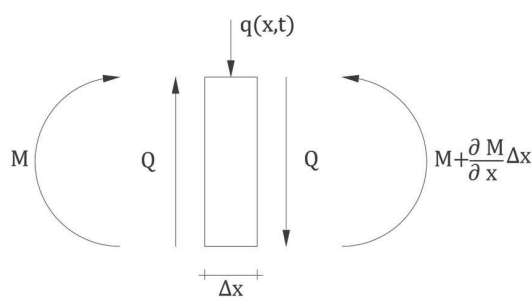

Equilibrio de momentos

Figura 3.1: Carga móvil sobre viga de sección variable. equilibrio de la rebanada

Planteando el equilibrio en la rebanada mediante el principio de D'Alembert, despreciando, en principio la contribución del amortiguamiento:

- equilibrio de fuerzas:

$$
-\frac{\partial Q(x, t)}{\partial x}-p_{0} \cdot \delta(x-v t)=\rho(x) \cdot \frac{\partial^{2} u}{\partial t^{2}}
$$

Donde el término de la derecha de la igualdad de la ecuación es la fuerza de inercia (eliminada en estática) y $\rho(x)$ la densidad lineal de la estructura.

- Equilibrio de momentos:

$$
\partial M(x, t)=Q(x, t) \partial x \Rightarrow \frac{\partial M(x, t)}{\partial x}=Q(x, t)
$$

Si sustituímos 3.3 en 3.2:

$$
\begin{gathered}
-\frac{\partial\left(\frac{\partial M(x, t)}{\partial x}\right)}{\partial x}-p_{0} \cdot \delta(x-v t)=\rho(x) \cdot \frac{\partial^{2} u}{\partial t^{2}} \\
\frac{\partial^{2} M(x, t)}{\partial x^{2}}-p_{0} \cdot \delta(x-v t)=\rho(x) \cdot \frac{\partial^{2} u}{\partial t^{2}}
\end{gathered}
$$

Como ecuación constitutiva, se adopta la relación lineal momento-curvatura del modelo de viga de Euler-Bernouilli:

$$
\frac{\frac{\partial^{2} u(x, t)}{\partial x^{2}}}{1+\left(\frac{\partial u}{\partial x}\right)^{2}} \approx \frac{\partial^{2} u(x, t)}{\partial x^{2}}=\frac{M(x, t)}{E I(x)}
$$




$$
M ; 0(x, t)=E I(x) \cdot \frac{\partial^{2} u(x, t)}{\partial x^{2}}
$$

Ecuación que una vez sustituída en 3.5, permite la obtención de la ecuación de gobierno del problema:

$$
\begin{gathered}
\frac{\partial E I(x) \cdot \frac{\partial^{2} u(x, t)}{\partial x^{2}}}{\partial x^{2}}-p_{0} \cdot \delta(x-v t)=\rho(x) \cdot \frac{\partial^{2} u}{\partial t^{2}} \\
\rho(x) \cdot \frac{\partial^{2} u}{\partial t^{2}}+\frac{\partial^{2}}{\partial x^{2}}\left[E I(x) \cdot \frac{\partial^{2} u(x, t)}{\partial x^{2}}\right]+p_{0} \cdot \delta(x-v t)=0
\end{gathered}
$$

Ecuación diferencial que debemos resolver supuestas condiciones iniciales de reposo:

$$
\begin{gathered}
u(s, 0)=0, \forall x \\
\left.\frac{\partial u(x, t)}{\partial t}\right|_{t=0}=0, \forall x
\end{gathered}
$$

Paso 2.Discretización espacial para un elemento barra.Formulación matricial para toda la estructura

Esta subsección va obtener la formulación matricial que rige el comportamiento dinámico de un elemento, formulación necesaria para obtener la formulación matricial acoplada para toda la estructura. Dada una viga de longitud L. según la figura 3.2 sometida a:

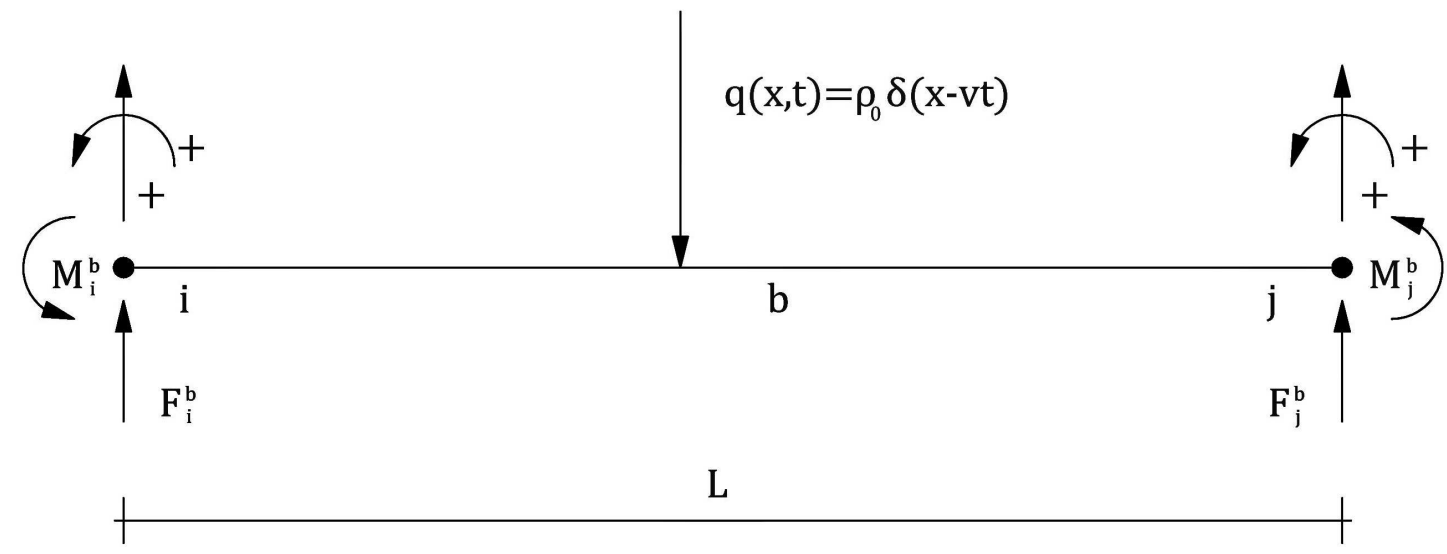

Figura 3.2: Discretización para un elemento barra y criterio de signos.

- Fuerzas externas:

1. Fuerzas y momentos nodales: Teniendo en cuenta el criterio de signos de la figura 3.2:

$$
\begin{gathered}
F_{i}^{b}=Q(0, t) \\
F_{j}^{b}=-Q(L, t)
\end{gathered}
$$




$$
\begin{gathered}
M_{i}^{b}=-M(0, t) \\
M_{j}^{b}=M(L, t)
\end{gathered}
$$

2. Fuerza puntual:

$$
p_{0} \cdot \delta(x-v t)
$$

3. Fuerzas de inercia:

$$
\rho(x) \cdot \frac{\partial^{2} u(x, t)}{\partial t^{2}}
$$

- Esfuerzos:

1. Cortantes:

$$
\frac{\partial Q(x, t)}{\partial x}=-p_{0} \cdot \delta(x-v t)-\rho(x) \cdot \frac{\partial^{2} u}{\partial t^{2}}
$$

Que integrándola ente 0 y $x \in[0, L]$ :

$$
Q(x, t)=Q(0, t)-\int_{0}^{x} p_{0} \cdot \xi(\xi-v t) d \xi-\int_{0}^{x} \rho(\xi) \cdot \frac{\partial^{2} u(\xi, t)}{\partial t^{2}} d \xi
$$

2. Flectores:

$$
\begin{gathered}
\frac{\partial M(x, t)}{\partial x}=Q(x, t) \cdot d x \\
M(x, t)=M(0, t)+\int_{0}^{x} Q(\xi, t) d \xi
\end{gathered}
$$

A continuación, se pasa a la formulación débil de la ecuación de Euler-Bernouilli de la viga multiplicando la ecuación de gobierno general 3.9 por una función de ponderación espacial, $u^{*}(x)$ y aplicando integración por partes. En lo que sigue se simplifica la notación para las derivadas de la siguiente forma:

$$
\begin{aligned}
& \frac{\partial u(x, t)}{\partial x}=\dot{u}(x, t) \\
& \frac{\partial u(x, t)}{\partial t}=\dot{u}(x, t)
\end{aligned}
$$

Se multiplica pues, 3.9 por $\mathrm{u}^{*}(x)$ :

$$
\begin{gathered}
\rho(x) \cdot \ddot{u}(x, t)+\left(u^{\prime \prime}(x, t) \cdot E I(x)\right)^{\prime \prime}=-p_{0} \cdot \delta(x-v t) \\
\rho(x) \cdot \ddot{u}(x, t) \cdot u^{*}(x)+\left(u^{\prime \prime}(x, t) \cdot E I(x)\right)^{\prime \prime} \cdot u^{*}(x)=-p_{0} \cdot \delta(x-v t) \cdot u^{*}(x)
\end{gathered}
$$

Integrando ene 1 dominio: 


$$
\begin{aligned}
& \int_{0}^{L} \rho(x) \cdot \ddot{u}(x, t) \cdot u^{*}(x) d x+\int_{0}^{L}\left(u^{\prime \prime}(x, t) \cdot E I(x)\right)^{\prime \prime} \cdot u^{*}(x) d x= \\
& =-\int_{0}^{L} p_{0} \cdot \delta(x-v t) \cdot u^{*}(x) d x(3.26)
\end{aligned}
$$

Aplicando doble integración por partes en el segundo sumando del primer término:

$$
\begin{aligned}
\int_{0}^{L}\left(u^{\prime \prime}(x, t) \cdot E I(x)\right)^{\prime \prime} \cdot u^{*}(x) d x= \\
=\left.\mathrm{u}^{*}(x) \cdot\left(u^{\prime \prime}(x, t) \cdot E I(x)\right)^{\prime}\right|_{0} ^{L}-\int_{0}^{L}\left(u^{\prime \prime}(x, t) \cdot E I(x)\right)^{\prime} \cdot \frac{\partial u^{*}(x)}{\partial x} d s= \\
=\left.\mathrm{u}^{*}(x) \cdot\left(u^{\prime \prime}(x, t) \cdot E I(x)\right)^{\prime}\right|_{0} ^{L}-\left.\left(u^{\prime \prime}(x, t) \cdot E I(x)\right) \cdot \frac{\partial u^{*}(x)}{\partial x}\right|_{0} ^{L}+ \\
+\int_{0}^{L} u^{\prime \prime}(x, t) \cdot E I(x) \cdot \frac{\partial^{2} u^{*}(x)}{\partial x^{2}}(3.27)
\end{aligned}
$$

Observando algunos de los sumandos de la expresión anterior se puede identificar fácilmente las expresiones de esfuerzos cortantes y momentos flectores:

$$
\begin{gathered}
Q(x, t)=\left(u^{\prime \prime} \cdot E I(x)\right)^{\prime} \\
M(x, t)=u^{\prime \prime} \cdot E I(x)
\end{gathered}
$$

Con lo cual, y teniendo en cuenta las relaciones anteriores, 3.28 y 3.29:

$$
\left.\left(u^{\prime \prime} \cdot E I(x)\right)^{\prime} \cdot u^{*}(x)\right|_{0} ^{L}=\left.Q(L, t) \cdot u^{*}(x)\right|_{0} ^{L}=
$$

$=\mathrm{Q}(\mathrm{L}, \mathrm{t}) \cdot u^{*}(L)-Q(0, t) \cdot u^{*}(0)=F_{j} b \cdot u^{*}(L)-F_{i}^{b} \cdot u^{*}(0)(3.30)$

$$
\begin{gathered}
\left.u^{\prime \prime} \cdot E I(s) \cdot \frac{\partial \cdot u^{*}(s)}{\partial s}\right|_{0} ^{L}=\left.M(s) \cdot \frac{\partial u^{*}}{\partial s}\right|_{0} ^{L}= \\
=-\left.M_{j}^{b} \cdot \frac{\partial u^{*}}{\partial s}\right|_{L}-\left.M_{i}^{b} \cdot \frac{\partial u^{*}}{\partial s}\right|_{0}(3.31)
\end{gathered}
$$

Con lo que la ecuación 3.27 resultaría:

$$
\begin{aligned}
& \int_{0}^{L}\left(u^{\prime \prime}(s, t) \cdot E I(s)\right)^{\prime \prime} \cdot u^{*}(s) d x= \\
& \int_{0}^{L} u^{\prime \prime}(s, t) \cdot E I(s) \cdot \frac{\partial^{2} u^{*}(s)}{2}-F_{j}^{b} \cdot u^{*}(L)-F_{i}^{b} \cdot u^{*}(0)-\left.M_{j}^{b} \cdot \frac{\partial u^{*}}{\partial s}\right|_{L} \\
& +\left.\mathrm{M}_{i}^{b} \cdot \frac{\partial u^{*}}{\partial s}\right|_{0}(3.32)
\end{aligned}
$$

En cuanto al segundo miembro de la ecuación 3.26, teniendo en cuenta las propiedades de la función Delta de Dirac:

$$
\int_{0}^{L} p_{0} \cdot \delta(x-v t) \cdot u^{*}(x) \cdot d x=p_{0} \cdot u^{*}(v t)
$$


De esta forma, sustituyendo las ecuaciones 3.32 y 3.33 en la ecuación 3.26 se obtiene:

$$
\begin{array}{r}
\int_{0}^{L} \rho(x) \cdot \ddot{u}(x, t) \cdot u^{*}(x) d x+\int_{0}^{L}\left(u^{\prime \prime}(x, t) \cdot E I(x)\right)^{\prime \prime} \cdot u^{*}(s) d x= \\
=+\mathrm{F}_{j}^{b} \cdot u^{*}(L)+F_{i}^{b} \cdot u^{*}(0)+\left.M_{j}^{b} \cdot \frac{\partial u^{*}}{\partial x}\right|_{L}+\left.M_{i}^{b} \cdot \frac{\partial u^{*}}{\partial x}\right|_{0}-p_{0} \cdot u^{*}(v t)(3.34)
\end{array}
$$

Expresión en la que no se ha impuesto condición alguna sobre $u^{*}(x)$ más que su continuidad y derivabilidad. Continuando con los conceptos básicos de elementos finitos, se considera una base de polinomios de grado menor o igual a 3 de Hermite, pues se han discretizado los elementos barra por dos nodos extremos con 4 grados de libertad, dos de desplazamientos y otros dos de giro. Se supone que $u(x, t)$ puede representarse de forma exacta como una combinación lineal de la base de los polinomios anteriormente indicada:

$$
u(x, t) \sum_{n=1}^{4} y_{n}(t) \cdot h_{n}(x)
$$

Siendo las expresiones de los polinomios de Hermite:

$$
\begin{gathered}
{\left[U_{i}\right] \longrightarrow h_{1}(x)=1-3 \cdot\left(\frac{x}{L}\right)^{2}+2 \cdot\left(\frac{x}{L}\right)^{3}} \\
{\left[\theta_{i}\right] \longrightarrow h_{2}(x)=L \cdot\left[\frac{x}{L}-2 \cdot\left(\frac{x}{L}\right)^{2}+\left(\frac{x}{L}\right)^{3}\right]} \\
{\left[U_{j}\right] \longrightarrow h_{3}(x)=3 \cdot\left(\frac{x}{L}\right)^{2}-2 \cdot\left(\frac{x}{L}\right)^{3}} \\
{\left[\theta_{j}\right] \longrightarrow h_{4}(x)=L \cdot\left[-\left(\frac{x}{L}\right)^{2}+\left(\frac{x}{L}\right)^{3}\right]}
\end{gathered}
$$

Desarrollando la ecuación 3.35 usando las relaciones anteriores:

$$
u(x, t)=y_{i} \cdot h_{1}(x)+\theta_{i} \cdot h_{2}(x)+y_{j} \cdot h_{3}(x)+\theta_{j} \cdot h_{4}(x)
$$

Si se deriva se puede obtener la velocidad y aceleración:

$$
\begin{gathered}
u(\dot{x}, t)=\dot{y}_{i} \cdot h_{1}(x)+\dot{\theta}_{i} \cdot h_{2}(x)+\dot{y}_{j} \cdot h_{3}(x)+\dot{\theta}_{j} \cdot h_{4}(x) \\
u(\dot{x}, t)=\sum_{n=1}^{4} \dot{y}_{n}(t) \cdot h_{n}(x) \\
u(\ddot{x}, t)=\ddot{y}_{i} \cdot h_{1}(x)+\ddot{\theta}_{i} \cdot h_{2}(x)+\ddot{y}_{j} \cdot h_{3}(x)+\ddot{\theta}_{j} \cdot h_{4}(x) \\
u(\ddot{x}, t)=\sum_{n=1}^{4} \ddot{y}_{n}(t) \cdot h_{n}(x)
\end{gathered}
$$


Si se toman como funciones de ponderación $u^{*}(x)$ las propias funciones de Hermite:

$$
\begin{aligned}
& \int_{0}^{L} \rho(x) \cdot \sum_{n=1}^{4}\left[y_{n}(t) \cdot h_{n}(x)\right] \cdot h_{m}(x) d x+\int_{n=1}^{4} \ddot{\sum}_{n=1}^{4}\left[y_{n}(t) \cdot \frac{\partial^{2} h_{n}(x)}{\partial x^{2}}\right] \cdot E I(x) h_{m}(x) \partial x^{2}= \\
= & +\mathrm{F}_{j}^{b} \cdot h_{m}(L)+F_{i}^{b} \cdot h_{m}(0)+\left.M_{j}^{b} \cdot \frac{\partial h_{m}(x)}{\partial x}\right|_{L}+\left.M_{i}^{b} \cdot \frac{\partial h_{m}(x)}{\partial x}\right|_{0}-p_{0} \cdot h_{m}(v t)(3.45)
\end{aligned}
$$

Se obtiene la formulación matricial:

$$
\left[\begin{array}{llll}
m_{11} & m_{12} & m_{13} & m_{14} \\
m_{21} & m_{22} & m_{23} & m_{24} \\
m_{31} & m_{32} & m_{33} & m_{34} \\
m_{41} & m_{42} & m_{43} & m_{44}
\end{array}\right]\left[\begin{array}{c}
\ddot{y}_{i}(v t) \\
\ddot{\theta}_{i}(v t) \\
\ddot{y}_{j}(v t) \\
\ddot{\theta}_{j}(v t)
\end{array}\right]+\left[\begin{array}{llll}
k_{11} & k_{12} & k_{13} & k_{14} \\
k_{21} & k_{22} & k_{23} & k_{24} \\
k_{31} & k_{32} & k_{33} & k_{34} \\
k_{41} & k_{42} & k_{43} & k_{44}
\end{array}\right]\left[\begin{array}{c}
y_{i}(v t) \\
\theta_{i}(v t) \\
y_{j}(v t) \\
\theta_{j}(v t)
\end{array}\right]=\left[\begin{array}{c}
F_{i}^{b}(v t) \\
M_{i}^{b}(v t) \\
F_{j}^{b}(v t) \\
F_{j}^{b}(v t)
\end{array}\right]-p_{0}\left[\begin{array}{c}
h_{1}(v t) \\
h_{2}(v t) \\
h_{3}(v t) \\
h_{4}(v t)
\end{array}\right]
$$

Donde:

$$
\begin{gathered}
M_{i j}=\int_{0}^{L} \rho(x) \cdot h_{i}(x) \cdot h_{j}(x) d x \\
K_{i j}=\int_{0}^{L} h_{i}^{\prime \prime}(x) \cdot E I(x) \cdot h_{j}^{\prime \prime}(x) d x
\end{gathered}
$$

Se trata de matrices en las que si se observan los subíndices, son simétricas. Si se acoplan estas matrices elementales es posible obtener una formulación global, par ala estructura completa:

$$
\mathbf{M} \ddot{\mathbf{y}}+\mathbf{K y}=-p_{0} \mathbf{h}(v t)
$$

\section{Paso 3.Resolución del sistema.}

Puesto que se trata de matrices simétricas, la forma más sencilla de resolución es intentar desacoplar el sistema. Para ello se realiza un cambio de base, mediante la matriz c de cambio de base (obtenida del problema de autovalores), del sistema B (que coincidirá con la base de vectores proipios) a la base canónica E:

$$
\left(-w^{2} \mathbf{M}+\mathbf{K}\right) \mathbf{y}(t)=0 \Rightarrow\left|\mathbf{K}-w^{2} \mathbf{M}\right|=0
$$

Problema de autovalores que proporcionará los vectores propios o modos de vibración que componen la matriz $\mathrm{C}$. Las coordenadas del vector $y$ se relacionan con las coordenadas del mismo en la base $\mathrm{B}$, mediante la matriz $\mathrm{C}$ de la siguiente forma:

$$
\begin{gathered}
y=C q \\
q=C^{-1} y
\end{gathered}
$$

Realizando dicho cambio de base en 3.49 y premultiplicando por la traspuesta de C se desacopla el sistema y se obtiene: 


$$
\mathbf{C}^{T} \mathbf{M C} \ddot{\mathbf{q}}+\mathbf{C}^{T} \mathbf{K C q}=-p_{0} \mathbf{C}^{T} \mathbf{h}(v t)
$$

De modo que las matrices de masa y rigidez, $\mathrm{M} \mathrm{y} \mathrm{K}$, resultan diagonalizadas:

$$
\begin{aligned}
& \mathbf{M}_{D}=\mathbf{C}^{T} \mathbf{M C} \\
& \mathbf{K}_{D}=\mathbf{C}^{T} \mathbf{K C}
\end{aligned}
$$

De modo que si premultiplicamos por $\mathbf{M}_{D}^{-1}$ podemos escribir:

$$
\begin{gathered}
\mathbf{M}_{D}^{-1} \cdot \mathbf{M}_{D} \ddot{\mathbf{q}}+\mathbf{M}_{D}^{-1} \cdot \mathbf{K}_{D} \cdot \mathbf{q}=-p_{0} \cdot \mathbf{M}_{D}^{-1} \cdot \mathbf{C}^{T} \mathbf{h}(v t) \\
\ddot{\mathbf{q}}+\mathbf{D} \cdot \mathbf{q}=\mathbf{G} \cdot \mathbf{h}(v t)
\end{gathered}
$$

Siendo $\mathbf{D}$ una matriz diagonal tal que $D_{i i}=w_{i}^{2}$, y la matriz G:

$$
\mathbf{G}=-p_{0} \cdot \mathbf{M}_{D}^{-1} \cdot \mathbf{C}^{T}
$$

De este modo hemos conseguido desacoplar el sistema, siendo resoluble para cada función $q_{i}(t)$ de forma independiente. Los valores propios de esta matriz $w_{i}^{2}$ permiten obtener las frecuencias naturales.

\section{Paso 4.Solución para cada modo.}

Analizando el término de carga:

- Para todo instante de tiempo, la carga puntual se encuentra sobre algún elemento o fuera del intervalo $[0, \mathrm{~L})$.

- En cada instante de tiempo, y para cada modo, intervienen cuatro coeficientes procedentes de la matriz G para definir la función de carga. Como funciones temporales, sólo 4 de ellas pueden combinarse en un punto inferior a un intervalo de la forma $[0, \mathrm{~L})$.

Si ahora se eneriquece la ecuación modal con un término de amortiguamiento, el problema planteado a resolver vienen dado por:

$$
\begin{gathered}
\ddot{q}_{n}(t)+2 \zeta w_{n} \dot{q}_{n}(t)+w_{n}^{2} \cdot q_{n}(t)=\sum_{m=1}^{4} G_{n m} h_{m}(v t) \\
\left.q_{i}(t)\right|_{t=0}=q_{i}(0) \\
\left.\dot{q}_{i}(t)\right|_{t=0}=\dot{q}_{i}(0)
\end{gathered}
$$

En la que $\zeta$ representa el factor de amortiguamiento, en el que se puede introducir un valor distinto para cada modo (más ajustado a la realidad pues es evidente pensar que se amortiguarán mejor los modos altos a los bajos), aunque en la práctica se suele optar por un amortiguamiento constante para cada modo por lo que en el presente documento se procederá de igual manera. 
Se considera una escala de referencia local para cada elemento, en el que el instante $t=0$ coincide con el instante en el que la carga entra en el elemento, coincidente con el punto de abcisa 0 . De este modo se evitan exponeneciales con términos fuera de rango.

Si observamos la ecuación 3.59, vemos que se trata de una ecuación diferencial lineal no homogénea por lo que la descompondremos en la suma de una solución particular más la solución homogénea:

$$
q(t)=q_{H}(t)+q_{p}(t)
$$

- Solución homogénea:

$$
\begin{gathered}
q_{H}(t)=e^{-\zeta w_{i} t}\left(C_{1} \cos \left(w_{i D} t\right)+C_{2} \sin \left(w_{i D} t\right)\right) \\
w_{i D}=w_{i} \sqrt{1-\zeta^{2}}
\end{gathered}
$$

- Solución particular:

Si se descompone el término no homogéneo en 3.59:

$$
p_{0}\left(G_{1} h_{1}(v t)+G_{2} h_{2}(v t)+G_{3} h_{3}(v t)+G_{4} h_{4}(v t)\right)
$$

Se observa que se trata de un polinomio de tercer grado por lo que la ecuación particular será de la forma:

$$
q_{p}(t)=\alpha_{0}+\alpha_{1} v t+\alpha_{2}(v t)^{2}+\alpha_{3}(v t)^{3}
$$

Cuyos coeficientes se obtienen sustituyendo la ecuación 3.59 y resolviendo el sistema:

$$
\begin{gathered}
\alpha_{0}=v^{3} \alpha_{01}+v^{2} \alpha_{02}+v \alpha_{03}+\alpha_{04} \\
\alpha_{1}=v^{2} \alpha_{11}+v \alpha_{12}+\alpha_{13} \\
\alpha_{2}=v \alpha_{21}+\alpha_{22} \\
\alpha_{3}=\alpha_{31} \\
{[1] \mapsto \alpha_{01}=-\frac{24 \zeta\left(2 \zeta^{2}-1\right)\left(2 G_{1}-2 G_{3}+\left(G_{2}+G_{4}\right) L\right)}{L^{3} w_{i}^{5}}} \\
{[2] \mapsto \alpha_{02}=-\frac{2\left(4 \zeta^{2}-1\right)\left(3 G_{1}-3 G_{3}+\left(2 G_{2}+G_{4}\right) L\right)}{L^{2} w_{i}^{4}}} \\
{[3] \mapsto \alpha_{03}=-\frac{2 G_{2} \zeta}{w_{i}^{3}}}
\end{gathered}
$$




$$
\begin{gathered}
{[4] \mapsto \alpha_{04}=-\frac{G_{1}}{w_{i}^{2}}} \\
{[5] \mapsto \alpha_{11}=\frac{6\left(4 \zeta^{2}-1\right)\left(2 G_{1}-2 G_{3}+\left(G_{2}+G_{4}\right) L\right)}{L^{3} w_{i}^{4}}} \\
{[6] \mapsto \alpha_{12}=\frac{4 \zeta\left(3 G_{1}-3 G_{3}+\left(2 G_{2}+G_{4}\right) L\right)}{L^{2} w_{i}^{3}}} \\
{[8] \mapsto \alpha_{21}=-\frac{6 \zeta\left(2 G_{1}-2 G_{3}+\left(G_{2}+G_{4}\right) L\right)}{L^{3} w_{i}^{3}}} \\
{[9] \mapsto \alpha_{22}=-\frac{4 \zeta\left(3 G_{1}-3 G_{3}+\left(2 G_{2}+G_{4}\right) L\right)}{L^{2} w_{i}^{2}}} \\
{[10] \mapsto \alpha_{31}=\frac{2 G_{1}-2 G_{3}+\left(G_{2}+G_{4}\right) L}{w^{3} w_{i}^{2}}}
\end{gathered}
$$

Estos diez elementos dependen de amortiguamiento, longitud del elemento, frecuencias naturales y los coeficientes $G_{i}$ que a su vez dependen de los modos de vibración, sin depender en ningún momento de la velocidad de paso de la carga por lo que se podrían determinar en un principio estos diez parámetros para cualquier malla completamente.

En último lugar nos restaría determinar los parámetros $C_{1}$ y $C_{2}$ de la solución homogénea a partir de la imposición de las condiciones iniciales:

$$
\begin{gathered}
q(0)=\alpha_{0}+C_{1} \\
C_{1}=q(0)-\alpha_{0} \\
\dot{q}(0)=-\zeta w_{i} C_{1}+w_{i D} C_{2}+\alpha_{1} v \\
C_{2}=\frac{\dot{q}(0)+\zeta w_{i} C_{1}-\alpha_{1} v}{w_{i D}}
\end{gathered}
$$

Por último, destacar que esta metodología para vigas es generalizable para otros casos genéricos, con la única imposición de derivabilidad de la línea de carga, como se puede ver en la publicación [35] en la que los autores aplican el método para el caso de cargas de Kirchhoff esviadas.

\subsection{Generalización y ejecución del método}

Como se ha observado en 3.63 y 3.66, los parámetros necesarios para la solución del sistema, $C_{i}, w_{i}, G_{i}$, pueden obtenerse mediante análisis modal. Para ello, se ha utilizado el software SAP2000, pudiéndose haber calculado mediante cualquier otro software de elementos finitos. Se puede interpretar la ecuación matricial 3.46, mediante un operador diferencial L: 


$$
\mathcal{L}\{u(\bar{x}, t), v(\bar{x}, t), w(\bar{x}, t)\}=-p_{0} \cdot \delta(s-v t)
$$

Siendo s, la coordenada curvilínea de la línea de carga $C^{1}$, se resuelve el sistema mediante separación de variables, espacial y temporal, a través de análisis modal que, gracias a la diagonalización de las matrices de rigidez y masa, desacopla todo el sistema:

$$
\mathbf{C}^{T} \mathbf{M C} \ddot{\mathbf{q}}+\mathbf{C}^{T} \mathbf{K C q}=-p_{0} \mathbf{C}^{T} \mathbf{h}(v t)
$$

La única posición impuesta por la formulación semianalítica ha sido la derivabilidad de la línea de carga introducida. Mediante el software SAP2000 se han seleccionado en cada modelo de elementos finitos, tanto la línea de carga como los puntos de postproceso de la estructura, que no son más que los puntos en los que se desea la información a posteriori.

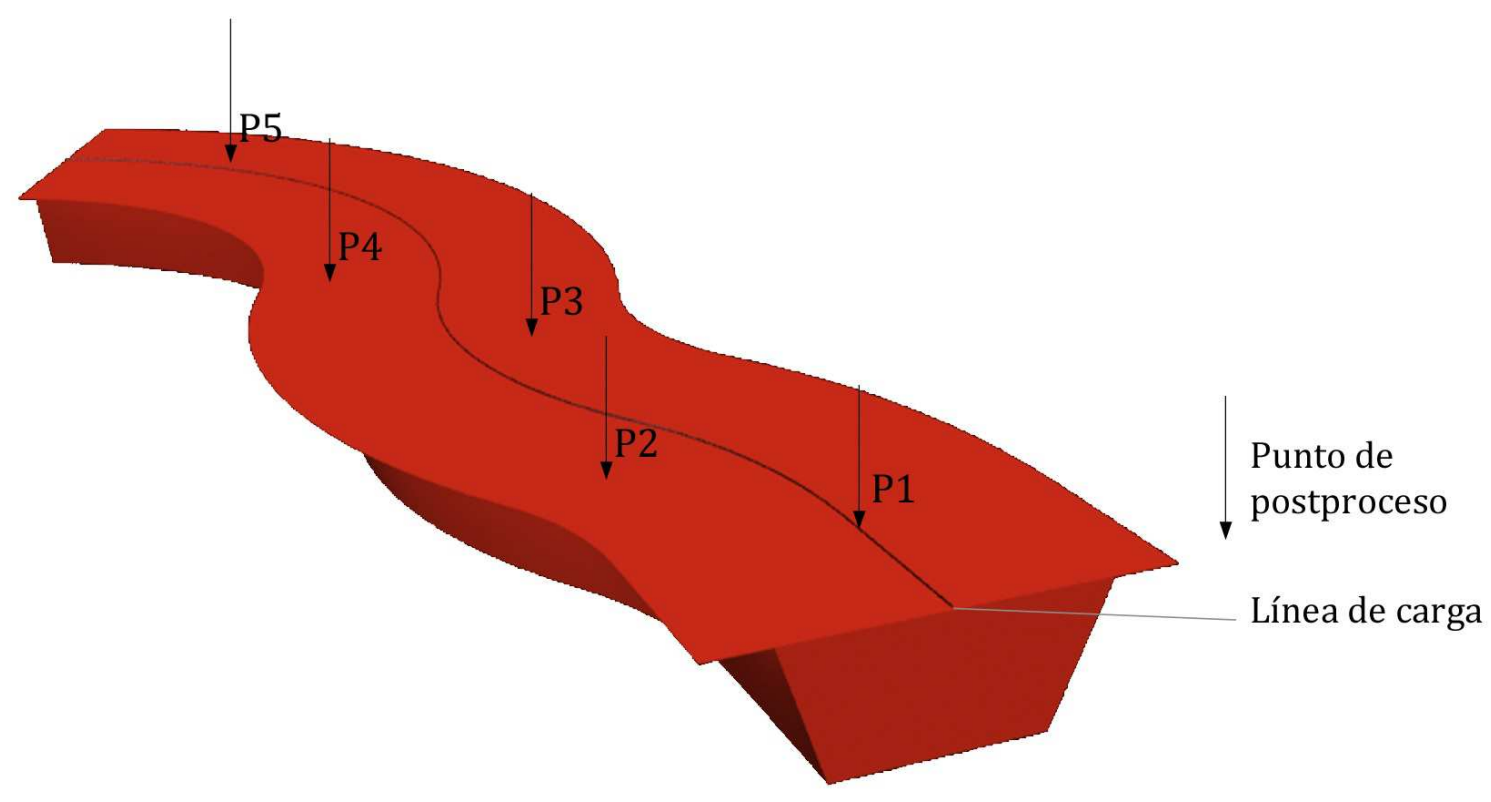

Figura 3.3: Esquema de línea de carga y puntos de postproceso en estructura.

La información espacial obtenida con el software SAP200 (los diez parámetros $\alpha_{n}$, así como las matrices $C$ y G) se proporcionan externamente al algoritmo semianalítico. Este algoritmo ha sido implememtado por el director de este proyecto en unentorno de cálculo intensivo basado en Linux y es donde se realiza la integración temporal del problema obteniendo la solución analítica (no aproximada en el tiempo) gracias al posible desacople des sistema, que es lo que ha permitido el tratamiento por separado de variable espacial y temporal.

\subsection{Test de validación}

\subsubsection{Planteamiento}

En este apartado se pretende contrastar numéricamente, los resultados arrojados mediante el método semianalítico frente a los métodos clásicos de integración paso a paso, más concretamente, el método de Newmark-Beta. Para ello se ha desarrollado un test de validación de un modelo bidimensional de vigas. 


\subsubsection{Test de validación 1: Viga de tres vanos}

Este primer test consiste en el contraste de resultados de ambos métodos sobre un modelo bidimensional de la estructura de tres vanos representada en la figura 3.4, caso analizado en publicaciones externas por Hayashikawa y Watanabe [17], Henchi et al. [18], Zheng et al. [36] y el director de este proyecto [2]:

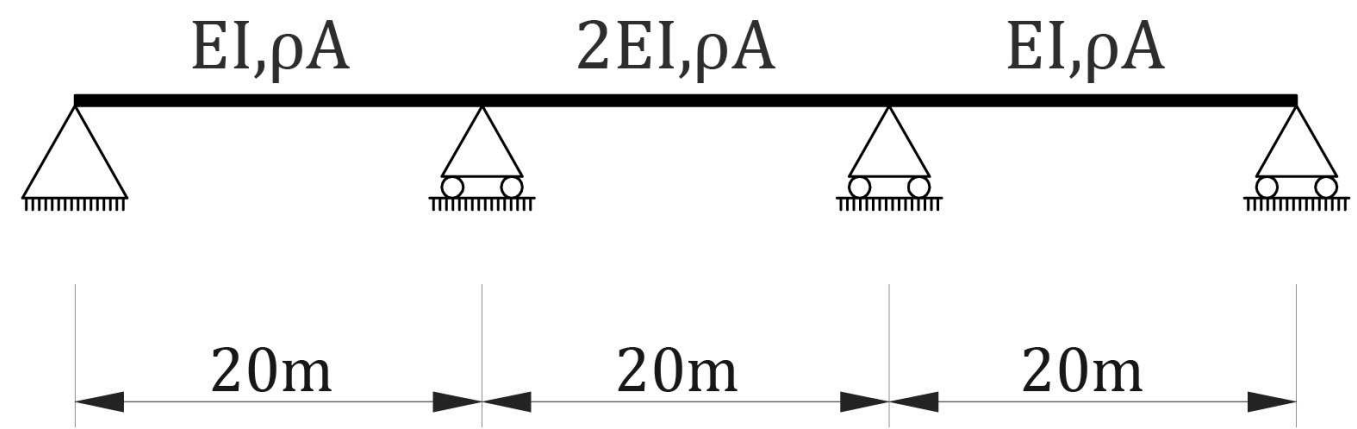

Figura 3.4: Geometría de la viga, Test1.

Las propiedades mecánicas de la estructura son:

- $L=20 \mathrm{~m}$ en todos los vanos.

- Rigidez a flexión $E I=1,96 G N m^{2}$ en los vanos laterales y $2 E I$ para el vano central.

- Masa por unidad de longitud $\rho \cdot A=1000 \mathrm{Kg} / \mathrm{m}$ constante para todos los vanos.

- $\xi=2 \%$ (amortiguamiento modal).

- Área a cortante nula para considerar un modelo de viga tipo Euler-Bernouilli.

Se considera una carga móvil aislada de valor $9.8 \mathrm{kN}$ desplazándose a una velocidad constante de $35.57 \mathrm{~m} / \mathrm{s}$.

Se realiza el cálculo modal de la estructura mediante el software SAP2000 para lo que se necesitará discretizar la estructura en 10 elementos por vano ya que el software SAP2000 establece su cálculo en base a la definición de un modelo de masas concentradas. Se consideran dos grados de libertad por nodo (desplazamiento y giro) y se trabaja con 12 modos de vibración. De esta forma se obtienen los siguientes cálculos: 


\begin{tabular}{|c|c|}
\hline Modo de vibración & Frecuencia \\
\hline 1 & 6,2044 \\
\hline 2 & 7,5812 \\
\hline 3 & 11,974 \\
\hline 4 & 24,204 \\
\hline 5 & 26,434 \\
\hline 6 & 37,281 \\
\hline 7 & 53,53 \\
\hline 8 & 56,585 \\
\hline 9 & 76,909 \\
\hline 10 & 93,844 \\
\hline 11 & 98,232 \\
\hline 12 & 129,98 \\
\hline
\end{tabular}

Cuadro 3.1: Frecuencias de modos vibración, Test1.

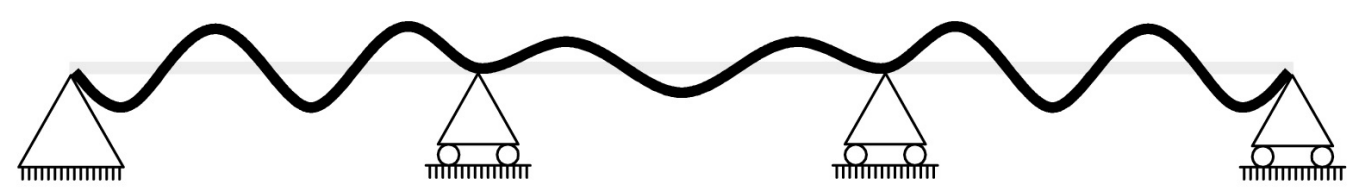

Figura 3.5: Ejemplo de modo e vibración 11, Test1.

Conocidas las frecuencias de vibración y las formas modales de la estructurase recurre al cálculo de la respuesta dinámica de la estructura al paso de la carga aislada. Para el cálculo mediante el método de Newmark (véase el apéndice A) se recurre de nuevo al software SAP2000 que implementa el métodod e Newmark tras un desacople modal del problema dinámico (metodología testada en la referencia [37]). Para el cálculo mediante el método semianalítico se recurre, sin embargo, al entorno de cálculo intensivo basado en Linux, preparado por el director de este proyecto.

\section{Cálculo mediante el método de Newmark:}

Como cualquier método de tipo incremental en el tiempo, se debe actuar sobre dos parámetros importantes del cálculo:

1. Tiempo de discretización de la carga.

2. Tiempo de discretización del paso o "step", que se hace coincidir con el anterior.

Se corre el cálculo con dos pasos de tiempo de $T_{12} / 25$ y $T_{12} / 150$ dónde $T_{12}$ es el período del modo 12:

$$
\begin{aligned}
& \frac{T_{12}}{25} \cong 3 E-04 s \\
& \frac{T_{12}}{150} \cong 5 E-05 s
\end{aligned}
$$


El objetivo es conseguir un paso lo suficientemente pequeño para ver si el método de Newmark es capaz de proporcionar un resultado parecido al semianalítico. Se obtiene de esta forma, los siguientes resultados para los primeros $0,1 \mathrm{~s}$ :

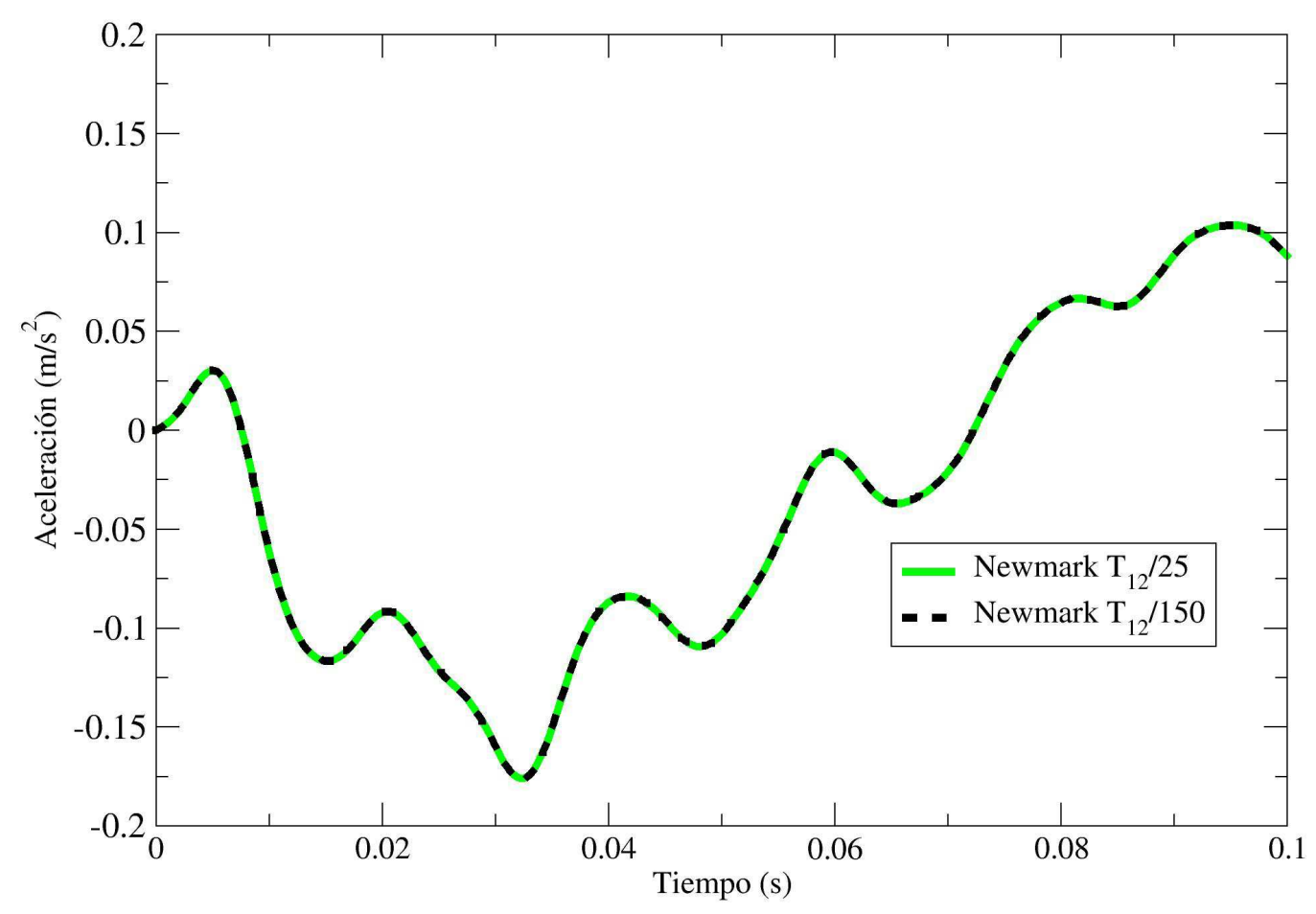

Figura 3.6: Aceleraciones en el punto central del primer vano por el métodod e Newmark. Test1.

Se ha presentado un problema destacable del software SAP2000 v14 que puede pasar desapercibido pero es de fácil reparación. Cada vez que éste software calcula una serie temporal, el resultado que otorga al inicio y final del primer paso de cálculo es nulo. Tras esto, la serie se calcula perfectamente pero retardada un paso de integración, con lo cual bastaría con hacer corresponder cada resultado con su valor de paso de tiempo debido un paso atrás.

\section{Cálculo mediante el Método semianalítico:}

Otra ventaja disponible con el método semianalítico es que conociendo las formas modale, pueden escogerse como puntos de postproceso para el análisis los que a priori pueden parecer más relevantes para el cálculo, mientras que el cálculo de Newmark mediante el software SAP2000 se realizará para los 31 nodos en los que se ha discretizado la estructura. Esto puede significar una reducción muy importante en los tiempos de cálculo para cualquier análisis de una estructura real de mayor complejidad, peues no tendrá 31 nodos, sino muchos más. Se escogen, por tanto: 


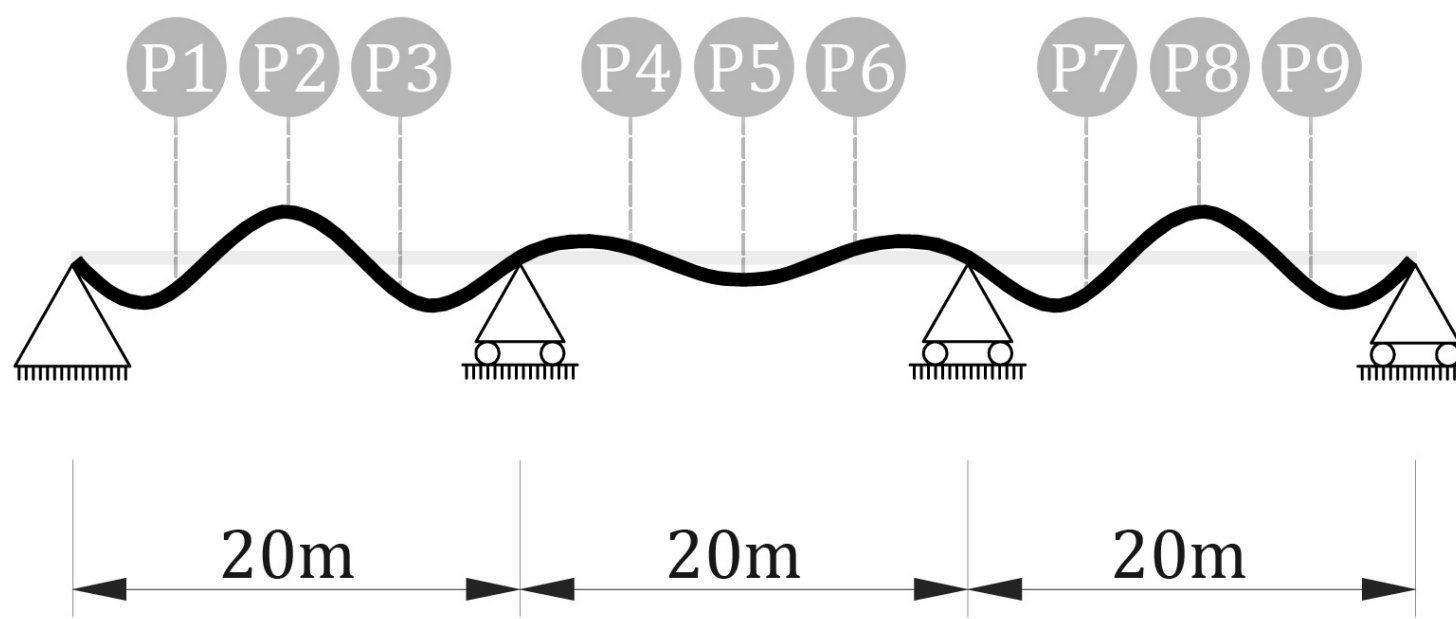

Figura 3.7: Puntos de postproceso de la estructura para el método semianalítico y modo de vibración 7.Test1.

En este caso, se lleva ahora el paso de cargas sobre la misma estructura con un paso de tiempo $\frac{T_{12}}{10}$ y se obtiene: 


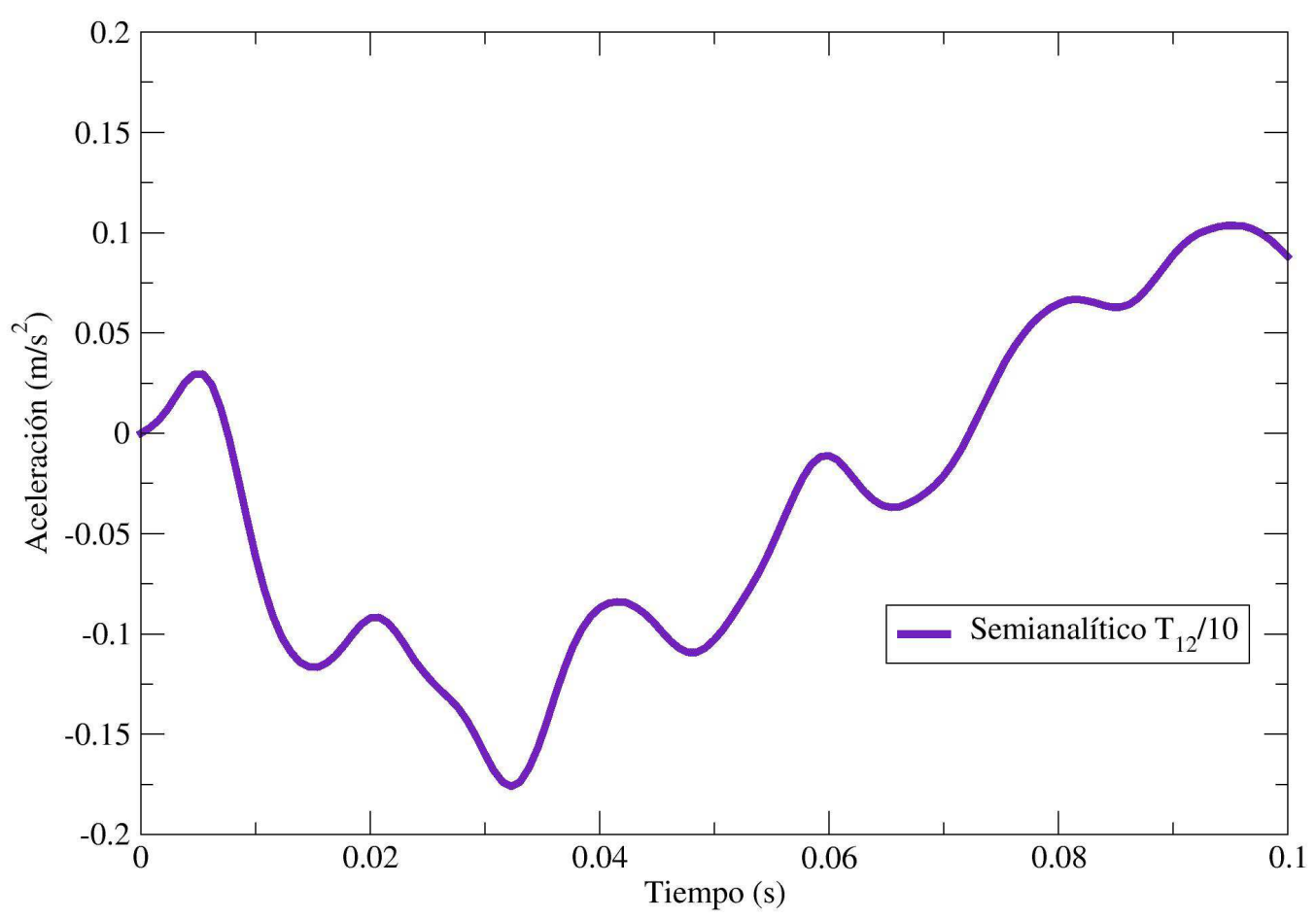

Figura 3.8: Aceleraciones en el punto central del primer vano por el método semianalítico.Test1.

\section{Comparativa:}

Se comparan las tres series temporales calculadas anteriormente tras reparar el contratiempo ocasionado por el software SAP2000 en el cálculo de la serie temporal por Newmark:

- Método Semianalítico de paso $\frac{T_{12}}{10}$.

- Método Newmark de paso $\frac{T_{12}}{25}$.

- Método Newmark de paso $\frac{T_{12}}{150}$. 


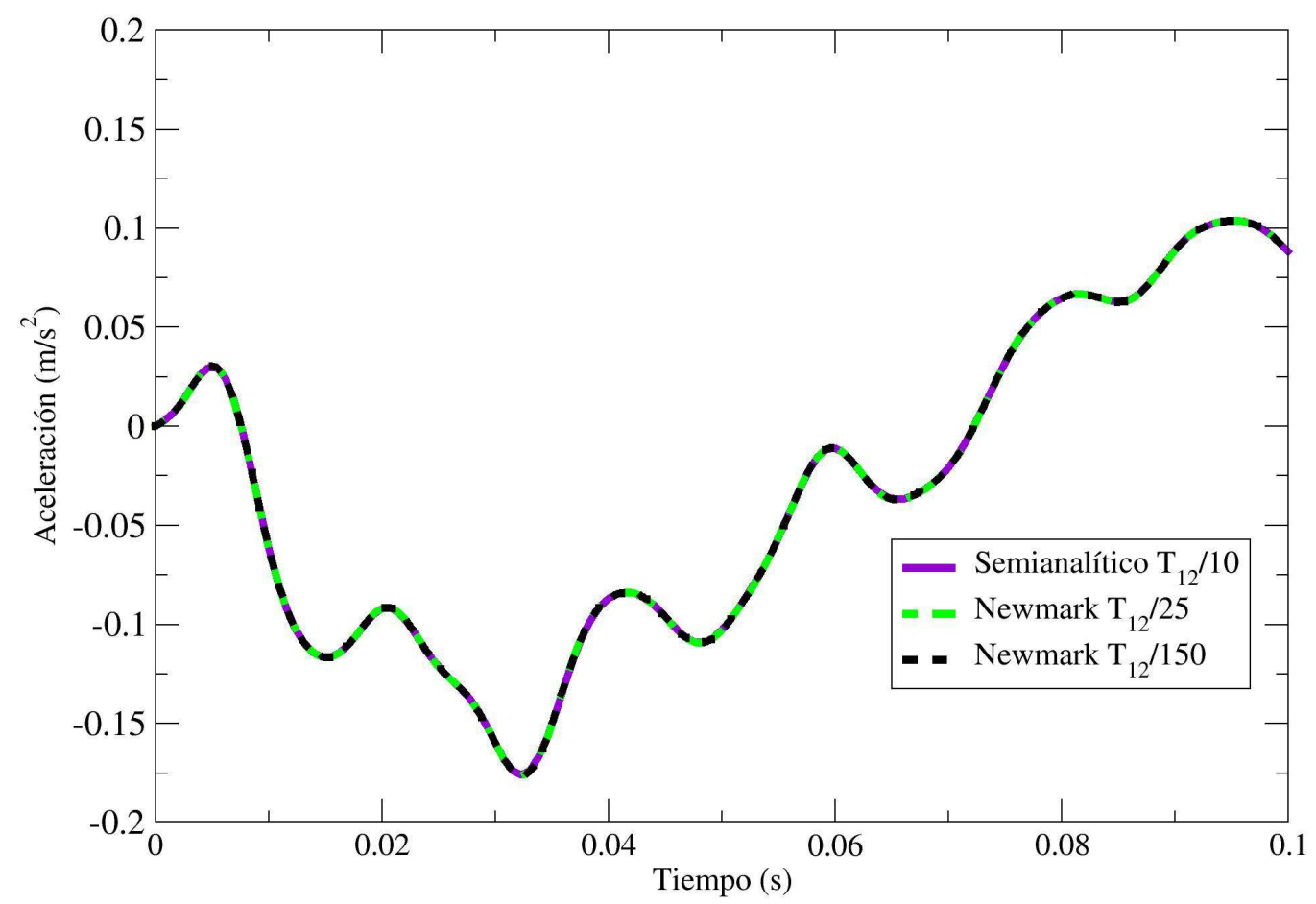

Figura 3.9: Comparación entre distintas metodologías de paso de cargas.Test1.

Se observa, pues, que el método semianalítico obtiene valores suficientemente aproximados a los métodos paso a paso en las fases iniciales de la serie, los primeros 0,1s. Estos datos coinciden con los publicados en las referencias [17], [18] y [36] y [2], por lo que se dan por buenos.

En la referencia [2], se puede consultar la Figura 5, donde se analiza la respuesta en fases posteriores, más concretamente entre 3,05 y 3,15 s. El objetivo de este cálculo es demostrar con resultados que la metodología de Newmark es de tipo incremental y que los errores se van acumulando conforme aumenta el número de pasos de cálculo (para conseguir información desde 0 hasta sólo 3,15 s se han necesitado hasta 61627 pasos de cálculo con uan duración por paso de $T_{12} / 150$ ). Como era de esperar, el método de Newmark se aproxima más a la solución exacta conforme más se refina el paso temporal de integración mientras que le método semianalítico sólo utiliza el muestreo de $t_{12} / 10$ para evaluar la respuesta cada cierto tiempo, no siendo la exactitud de los resultados dependiente de esta decisión. 


\subsection{Conclusión. Ventajas del método semianalítico}

Las ventajas de este método se desligan principalmente de la naturaleza analítica de la solución la cual siempre será mejor que cualquier aproximación. De modo que podremos enumerar:

1. El método proporciona resultados precisos pues las ecuaciones temporales se resuelven de forma analítica, por lo que se evitan los errores clásicos en el dominio del tiempo por la discretización del mismo por parte de los métodos de integración paso a paso. La solución se obtiene a partir de 10 coeficientes por elemento y modo que no dependen de la velocidad, lo cual facilita enormemente la aplicación de la actual norma IAPF que impone la obtención de resultados para un amplio rango de velocidades.

2. A bajas velocidades se converge a la solución estática, evitando el fenómenos de Gibbs típicamente asociado a las soluciones analíticas basadas en series armónicas.

3. Gracias a la normalización temporal se evitan problemas de exponenciales con argumentos elevados con los errores de sesgo que estos conllevan.

4. Se requiere de un paso de tiempo de evaluación, frente a un paso de tiempo de integración asociado a métodos paso a paso, en general mucho menor que el requerido para el muestreo analítico de la solución. Por ello, se gana velocidad de cálculo, ya que para tener una precisión similar al método semianalítico, los métodos paso a paso requieren más operaciones de punto flotante globales (debido a que el paso de tiempo es menor). La respuesta proporcionada en un tiempo dado por la solución semianalítica no depende del paso del tiempo; en métodos aproximados paso a paso sí depende.

5. Esta ventaja en ahorro de tiempo de cálculo convierte al método semianalítico en una herramienta práctica y realista de aplicación de las exigencias de la norma IAPF-2007. 


\section{CAPÍTULO 4}

\section{Análisis dinámico del Viaducto sobre el Tajo}

\subsection{Introducción}

El Viaducto sobre el río Tajo en el embalse de Alcántara es un puente arco continuo de vía doble, resuelto mediante una sección de cajón unicelular. La distribución de vanos se ve influenciada por el cruce del río Tajo, que se lleva a cabo mediante un arco de $324 \mathrm{~m}$ de longitud, dividiendo el tablero sobre el arco en seis vanos de $54 \mathrm{~m}$ cada uno. Los vanos de aproximación son de $60 \mathrm{~m}$, insertándose dos vanos de transición de $57 \mathrm{~m}$.

El puente tiene una longitud total de $1488 \mathrm{~m}$ con una distribución de luces 45-9x60-57-324-577x60-45 m. La estructura es continua, existiendo un único punto fijo, en el centro del arco".

En una estructura de este tipo el paso de cargas móviles excéntricas produce deformaciones simultáneas de flexión y torsión. Como es sabido, en determinados modos de vibración se producirá además un acoplamiento entre torsión y flexión lateral. Por estos motivos, el Cálculo Dinámico del Viaducto sobre el río Tajo se realiza mediante un método semianalítico que realiza la integración analítica (exacta) de las ecuaciones modales de movimiento de todos los modos cuya contribución a la respuesta sea necesario tener en cuenta. Los modos de vibración se obtienen mediante la técnica de los Elementos Finitos.

\subsection{Método de Cálculo}

Los fundamentos teóricos del método se publicaron en 2006 en la revista Journal of Sounds and vibration y que han sido desarrollados en el capítulo anterior.

Como se indicaba anteriormente, los modos de vibración se obtienen de un modelo de elementos finitos que se describirá en el apartado siguiente.

Para llevar a cabo el cálculo por superposición modal se tienen en cuenta las contribuciones de los modos de frecuencia menor o igual a $30 \mathrm{~Hz}$, que para el valor de balasto incrementado un $30 \%$ son 688. Este elevado número se debe en gran parte a modos asociados a flexiones que afectan fundamentalmente a los voladizos del tablero.

El paso de tiempo elegido para evaluar la historia temporal de la vibración del puente, ante la circulación de cada tren y a cada velocidad de paso, es la décima parte del menor período de vibración. La frecuencia máxima considerada es de $29.979 \mathrm{~Hz}$, como se indica en el apartado 4.6.1, por lo que el paso de tiempo para evaluación es de $0.003335 \mathrm{~s}$.

Las vibraciones libres que tienen lugar tras el paso del tren sobre la estructura se calculan durante un tiempo total igual a 6 períodos de vibración completos del modo fundamental, lo cual se considera suficiente para que se hayan reducido los niveles máximos de oscilación.

La tasa de amortiguamiento empleada para todos los modos es del $2 \%$, de acuerdo con la IAPF07. 


\subsection{Modelo numérico de la estructura}

El modelo empleado es del tipo lámina plegada, resuelto de manera aproximada mediante el método de los elementos finitos. Se considera la sección cajón del viaducto como un perfil cerrado formado por cuatro tipos de láminas. Los voladizos, las losas superior e inferior y las almas. Dichas láminas se discretizan mediante elementos finitos planos de cuatro nudos y seis grados de libertad por nudo. No se tiene en cuenta la deformación debida a las tensiones tangenciales en sentido perpendicular al plano medio de las láminas. Las riostras existentes en las zonas de apoyo en pilas y estribos se modelizan adecuando los espesores de las láminas de manera que se reproduce la masa total de las riostras, y añadiendo además restricciones cinemáticas en ciertas secciones que eliminan el alabeo y la distorsión. La rigidez de la zona resulta de ese modo comparativamente muy superior a la de la sección cajón.

El arco está constituído por un modelo de viga dividido en tres tramos con sección variable.

La figura 4.1 muestra un detalle de la malla de elementos finitos en la zona del estribo inicial, mientras que en la figura 4.2 se muestra el modelo de un grupo de riostras. En la figura 4.3 se observa la sección recta del modelo y en las figuras 4.4 y 4.5 el modelo de la estructura completa y un detalle del modelo del arco.

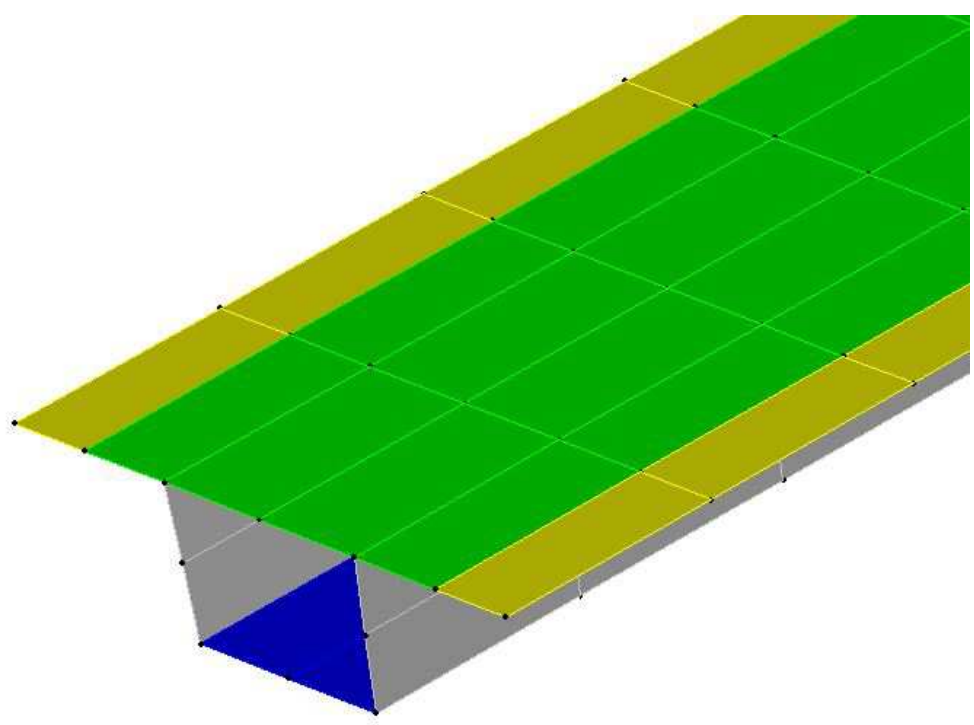

Figura 4.1: Detalle de la malla empleada. Sección del estribo inicial 



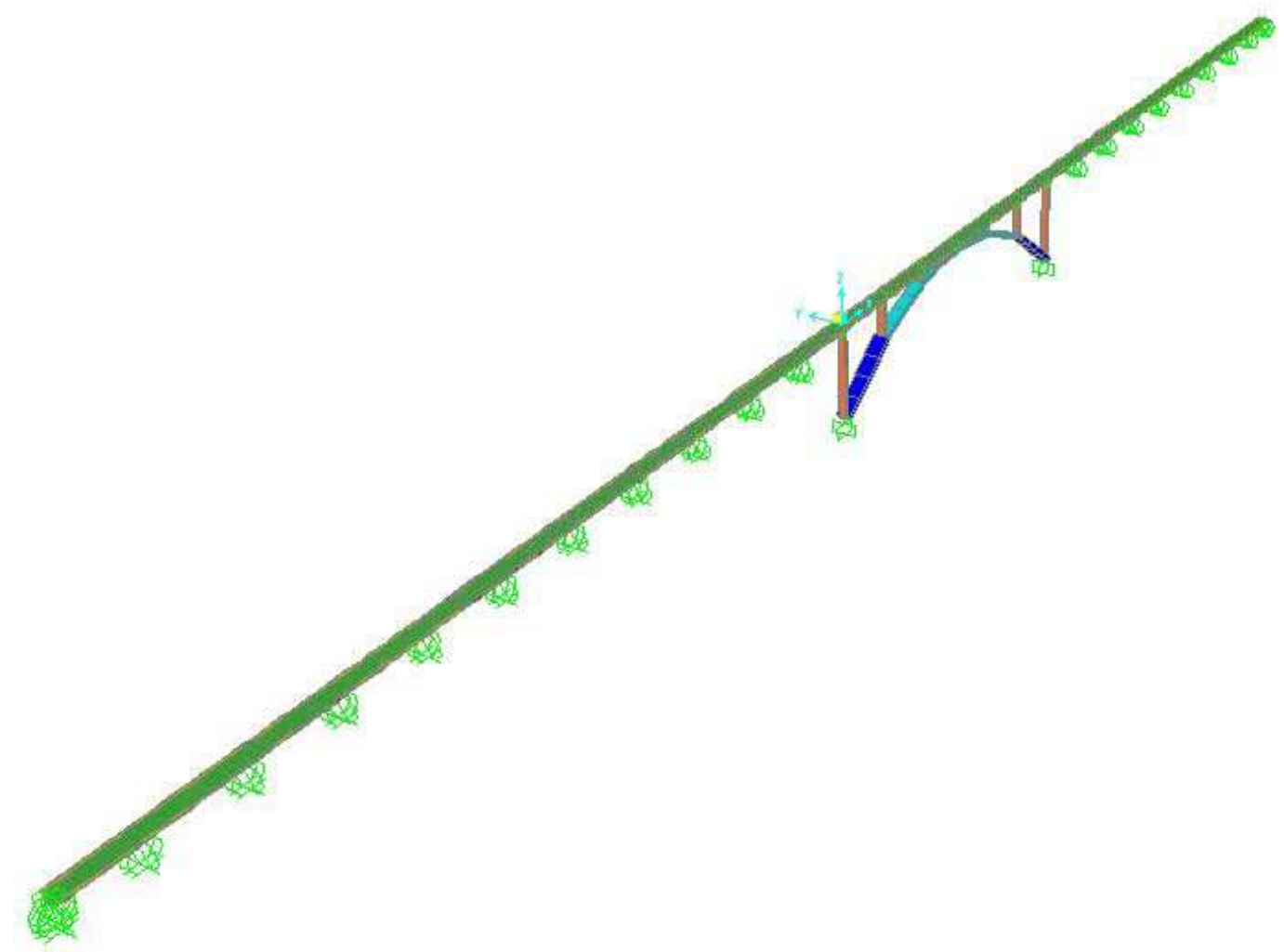

Figura 4.4: Modelo completo de la estructura

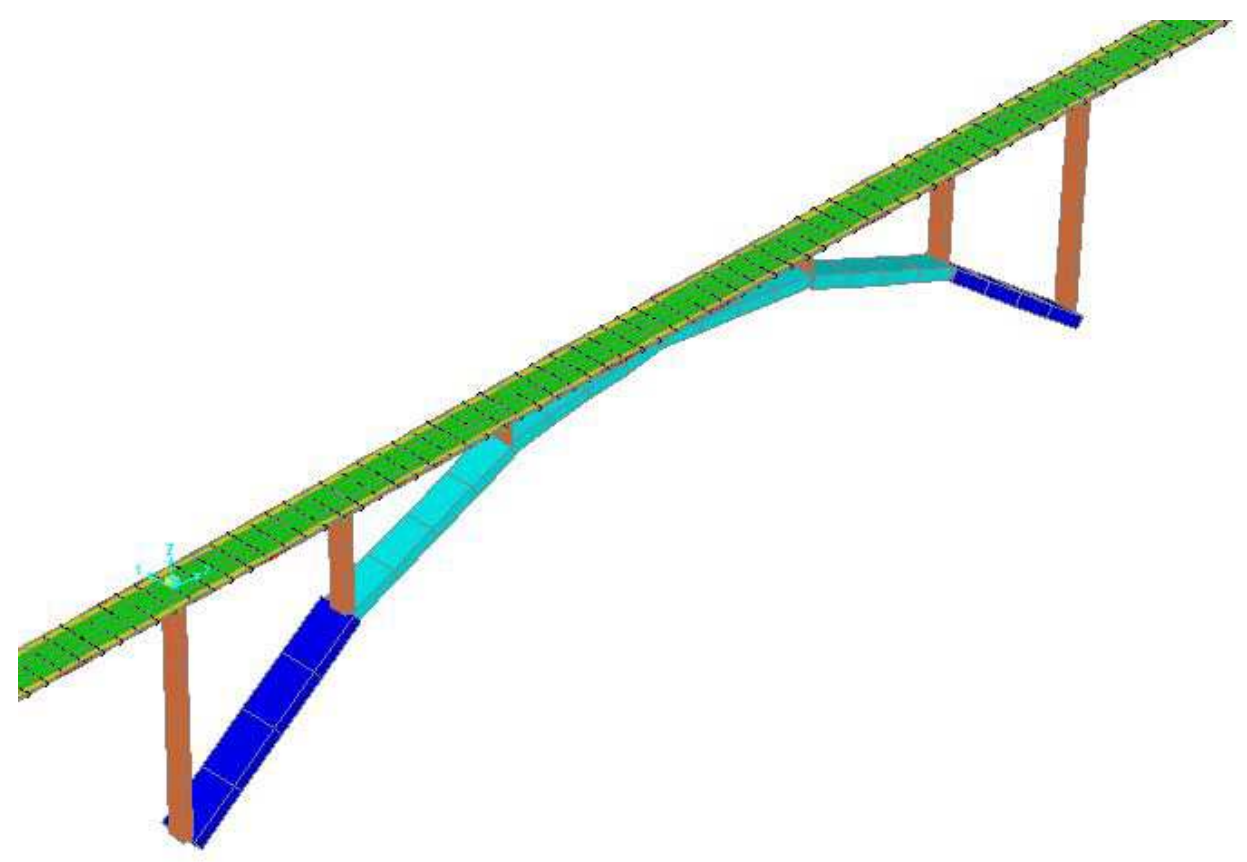

Figura 4.5: Modelo tipo viga del Arco 
Los espesores empleados para las distintas partes de la sección general, entendiendo por tal la que representan los 26 vanos en la mayor parte de su longitud, se deducen a partir de los planos de proyecto y son los siguientes:

Voladizos : variable entre 0.2 y $0.36 \mathrm{~m}$.

Losa Superior: $0.35 \mathrm{~m}$.

Losa Inferior: $0.3 \mathrm{~m}$.

Almas: $0.5 \mathrm{~m}$.

En todos los vanos existen, en los apoyos en pila y en la cercanía de estos, unas zonas de mayor espesor en las almas. En las figuras 4.6 a 4.9 se muestra la ubicación de las zonas en las existen espesores distintos a los de la sección general.

Las uniones entre almas y losas, de espesor variable, presentan dificultades para su modelización mediante elementos lámina. Por ese motivo se ha preferido mantener constante el espesor de las almas y losas del modelo, verificando posteriormente que la inercia de la sección y la masa lineal se reproducen con buena aproximación.

A partir de los planos de proyecto se determina que el área de la sección transversal es de $9.31 \mathrm{~m}^{2}$ y la incercia con respecto del eje principal horizontal es de $64.27 \mathrm{~m}^{4}$. Con la sección que se muestra en la figura 4.3 y los espesores indicados anteriormente, el cálculo como perfil de pared delgada produce un área de $9.17 \mathrm{~m}^{2}$ y una inercia de $64.49 \mathrm{~m}^{4}$, teniéndose por tanto una buena aproximación del área y de la rigidez a flexión de la sección.

Propiedades de los materiales:

Módulo de elasticidad: 38 GPa.

Coeficiente de Poisson: 0.3

Densidad: $2500 \mathrm{Kg} / \mathrm{m}^{3}$.

Cargas permanentes:

Sobre la losa superior y los voladizos descansa la masa correspondiente a los siguientes elementos:

Balasto: $13437 \mathrm{Kg} / \mathrm{m}$ (valor superior).

Vías y traviesas: $1280 \mathrm{Kg} / \mathrm{m}$ (comprende las dos vías). 
Muretes guardabalasto: $500 \mathrm{~kg} / \mathrm{m}$ (comprende los dos lados).

Aceras y canalizaciones: $2450 \mathrm{~kg} / \mathrm{m}$ (comprende los dos lados).

Barandilla e imposta: $900 \mathrm{Kg} / \mathrm{m}$ (comprende los dos lados). 


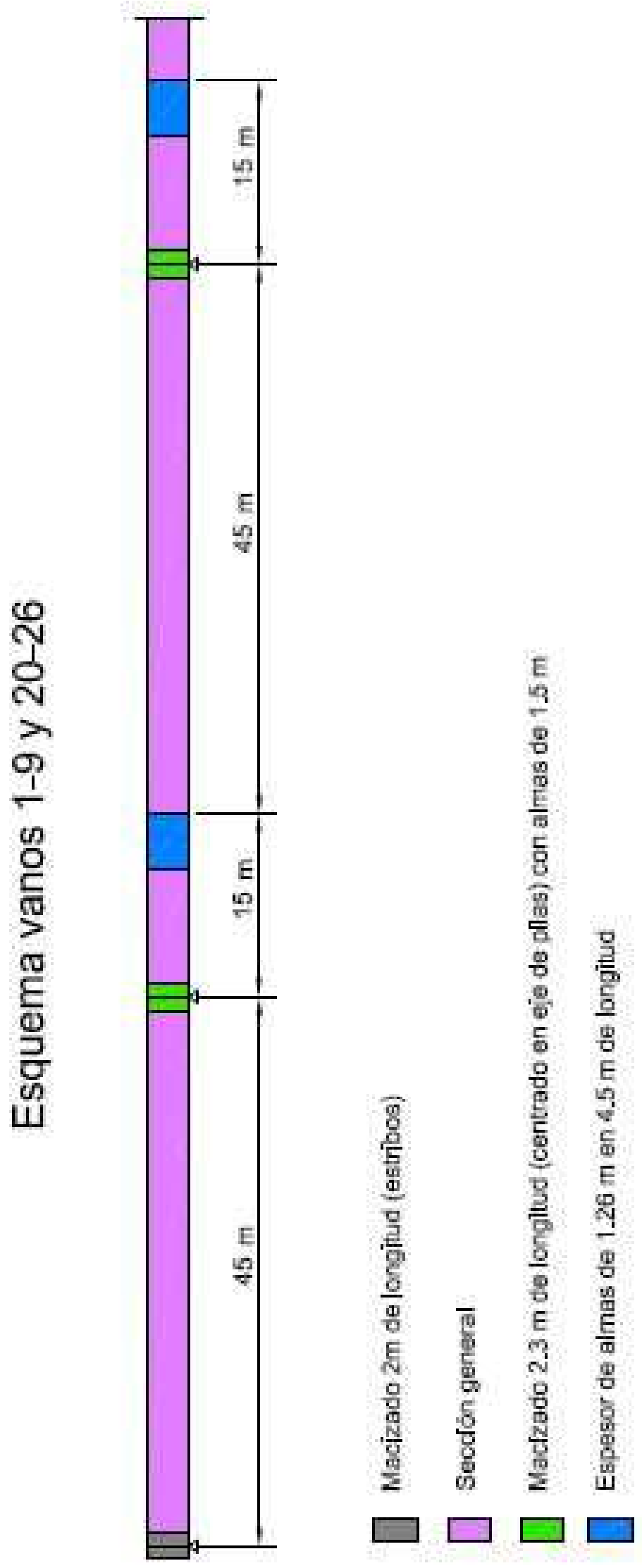

Figura 4.6: Alzado lateral con los distintos tipos de sección. Vanos 1-9 y 20-26 


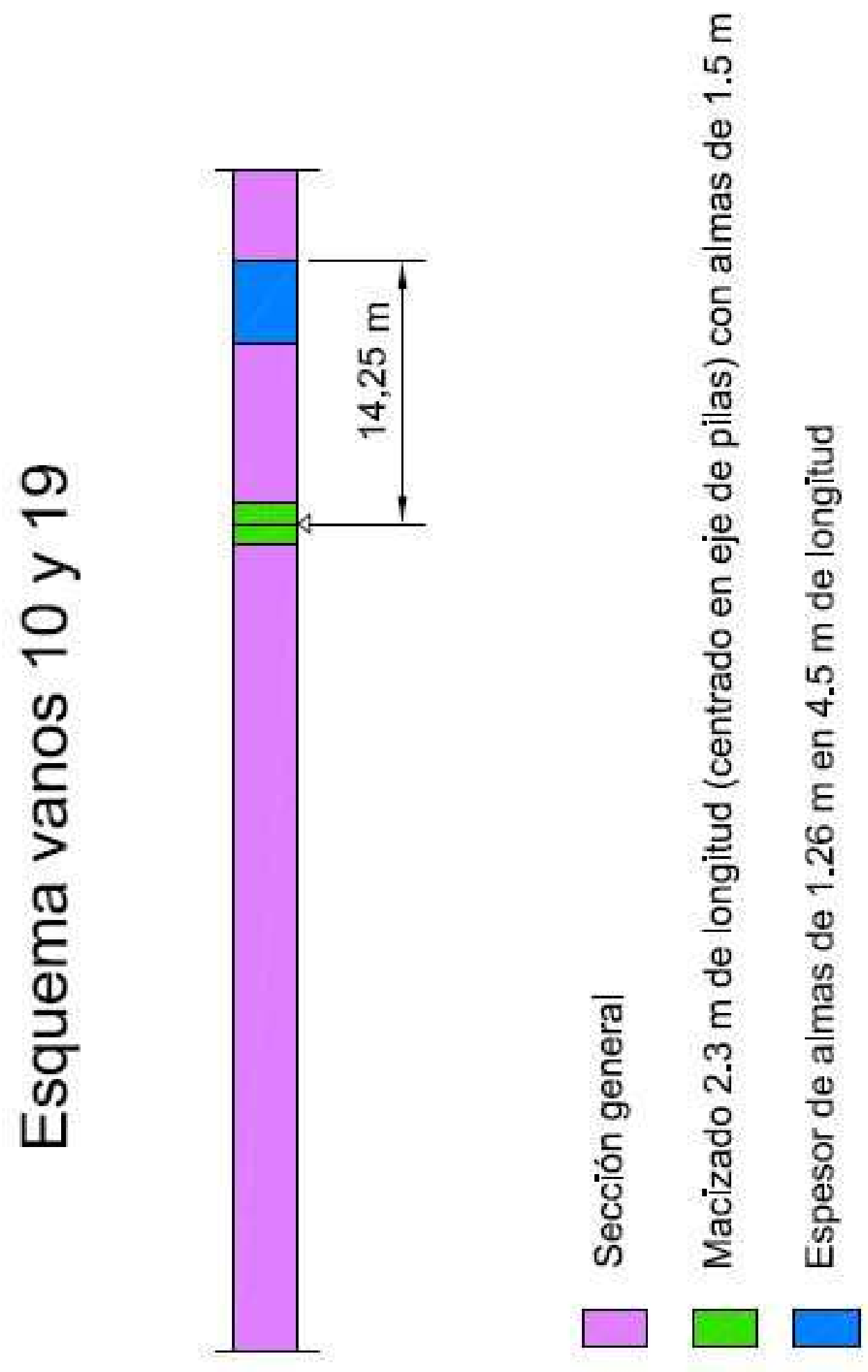

Figura 4.7: Alzado lateral con los distintos tipos de sección. Vanos 10 y 19 

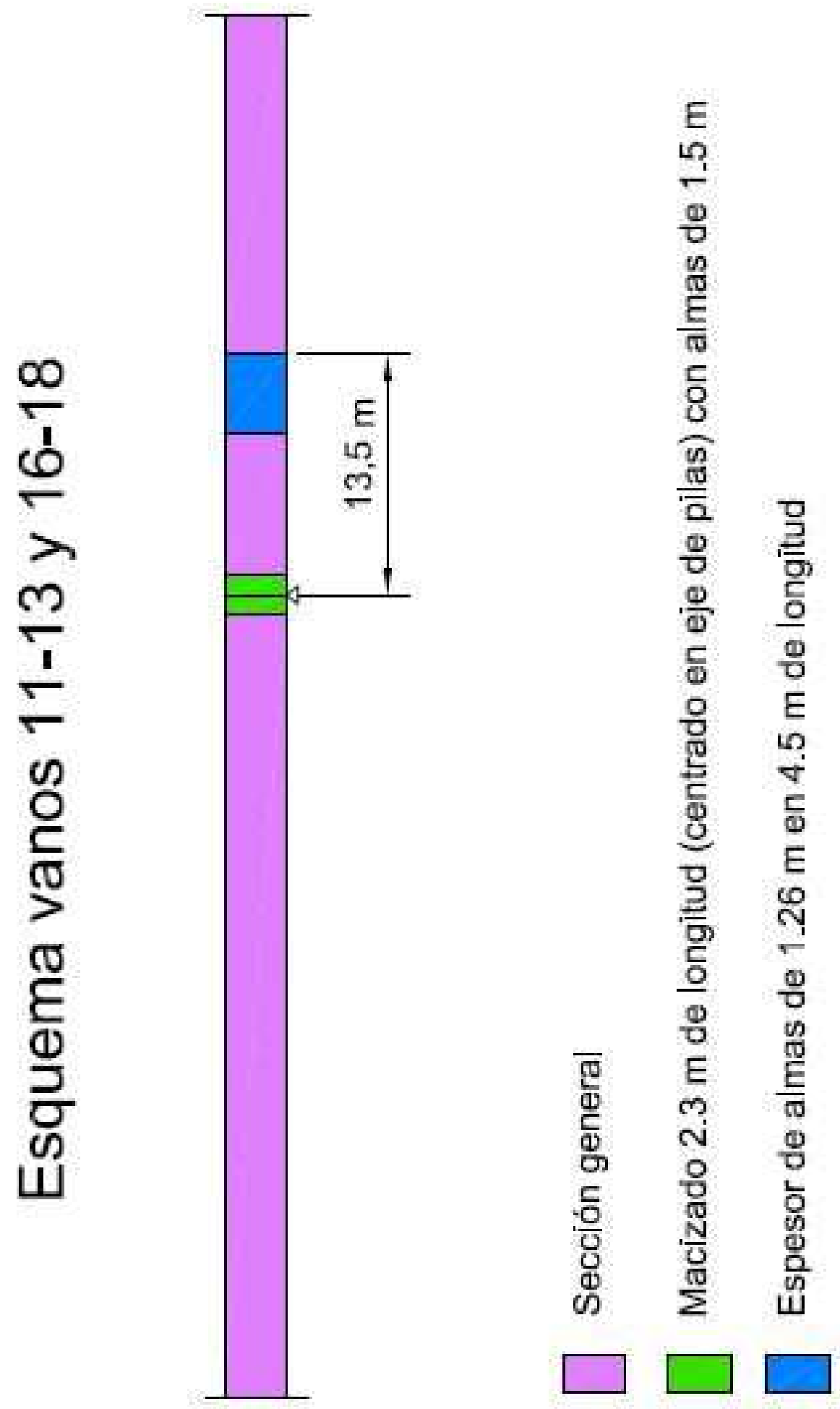

Figura 4.8: Alzado lateral con los distintos tipos de sección. Vanos 11-13 y 16-18 


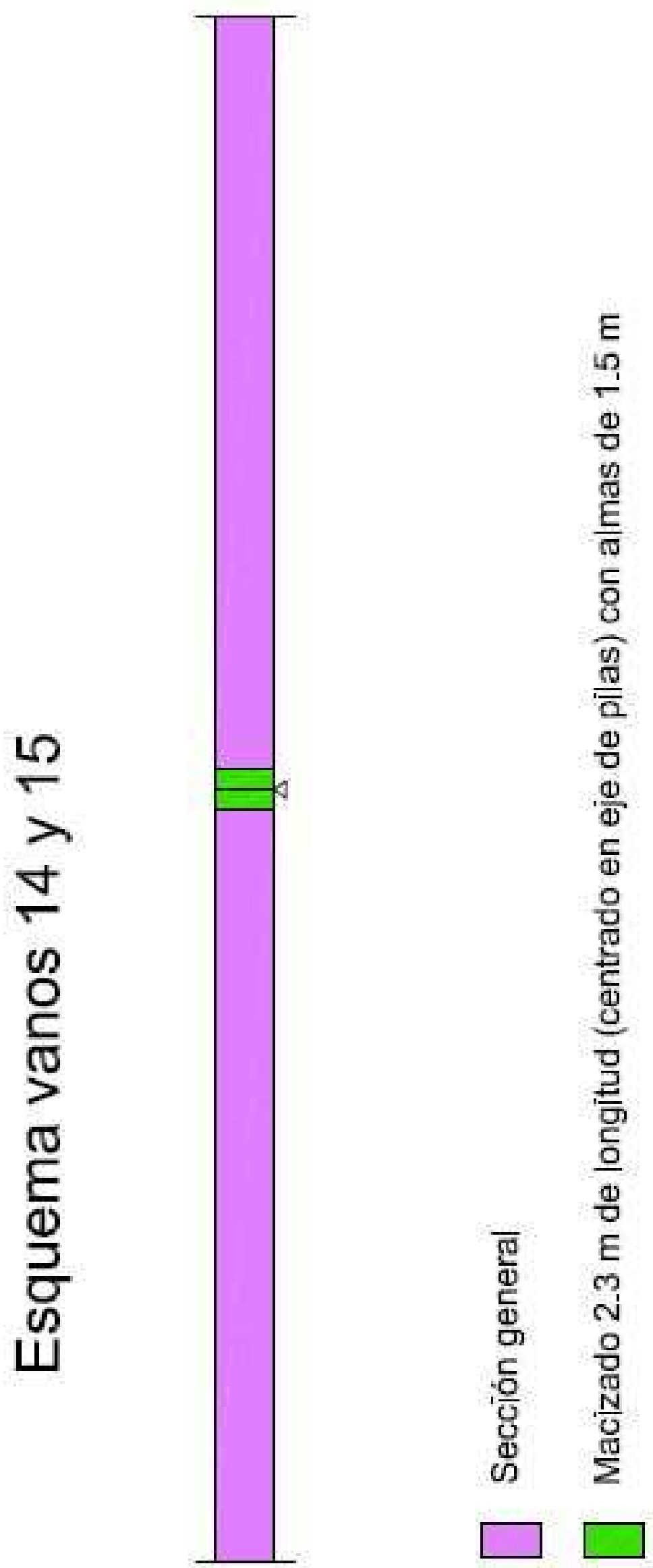

Figura 4.9: Alzado lateral con los disțintos tipos de sección. Vanos 14 y 15 


\section{Apoyos:}

Para generar el modelo de elementos finitos se emplea el sistema de coordenadas en el que el eje $X$ es el longitudinal, el $\mathrm{Y}$ el transversal horizontal y el eje $\mathrm{Z}$ el vertical. El origen se encuentra en el punto medio de la losa superior correspondiente a la sección del vano 12 donde comienza el arco.

Los apoyos de la estructura sobre estribos y pilas se modelizan mediante apoyos simples verticales dispuestos en las secciones correspndientes. La distancia entre ellos es por tanto igual a la longitud de los vanos. En cada una de las secciones, los apoyos verticales se sitúan en los puntos de unión entre almas y losa inferior, habiendo por tanto en total 54 apoyos verticales. Los movimientos de los puntos situados en el margen izquierdo se han restringido también en la dirección transveral Y. Además, se impide el movimiento en la dirección longitudinal $\mathrm{X}$ del punto central de la riostra situada en el primer estribo del puente.

En cuanto a los apoyos situados en los vanos sobre el arco, éstos se modeizan mediante links que simulan los apoyos esféricos sobre las pilas del arco. Las figuras 4.10, 4.11 y 4.12 muestran los puntos vinculados y las direcciones de dichos vínculos.

\section{Riostras:}

En los apoyos en pilas y estribos existen zonas macizadas de $5 \mathrm{~m}$ de longitud y $1 \mathrm{~m}$ de espesor, que confieren una rigdez muy superior lo cual impide el alabeo y la distorsión de la zona macizada. 

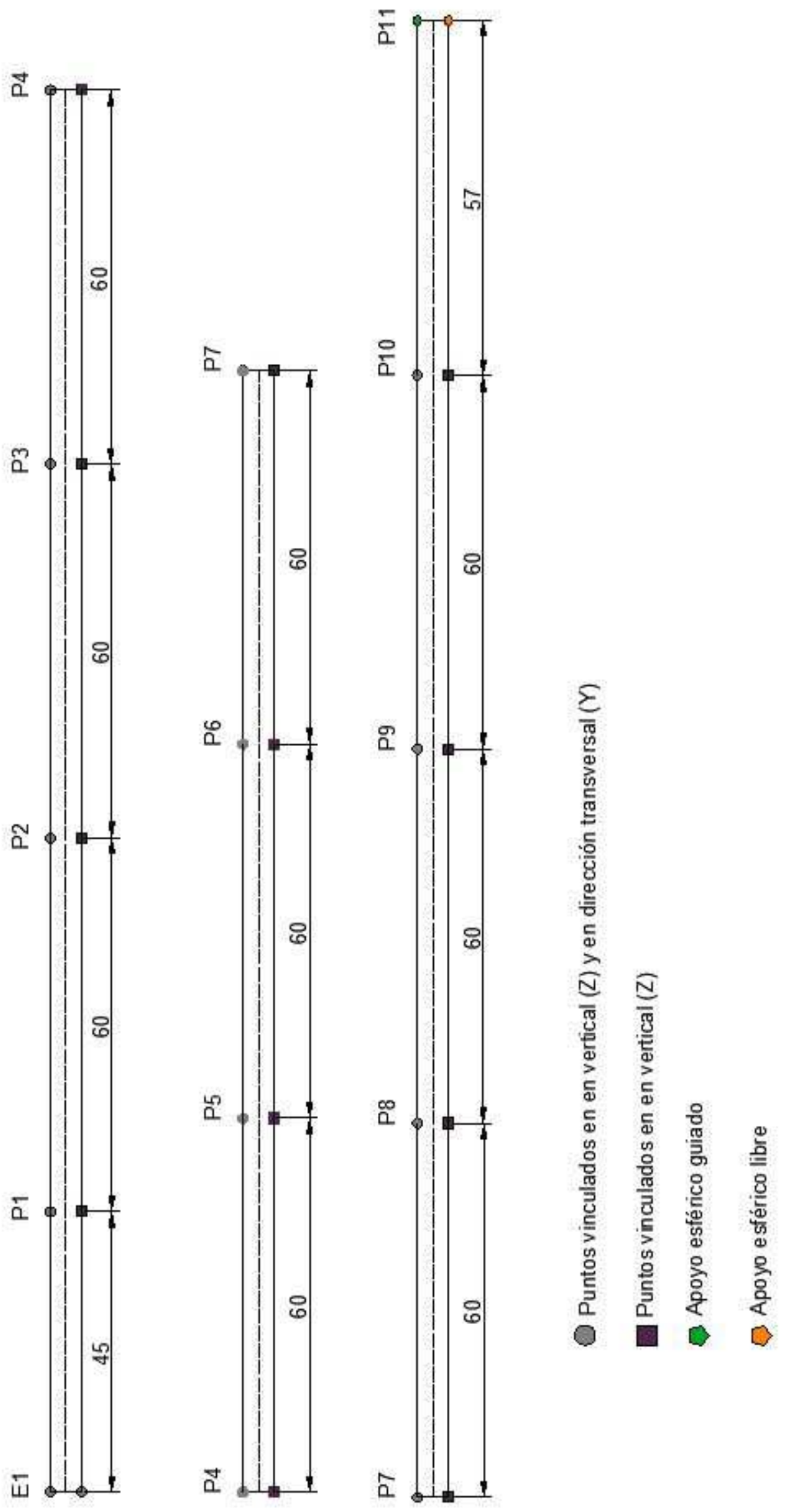

Figura 4.10: Vista en planta de la losa inferior. Sitų̧ción y dirección de los apoyos de los primeros 11 vanos. 


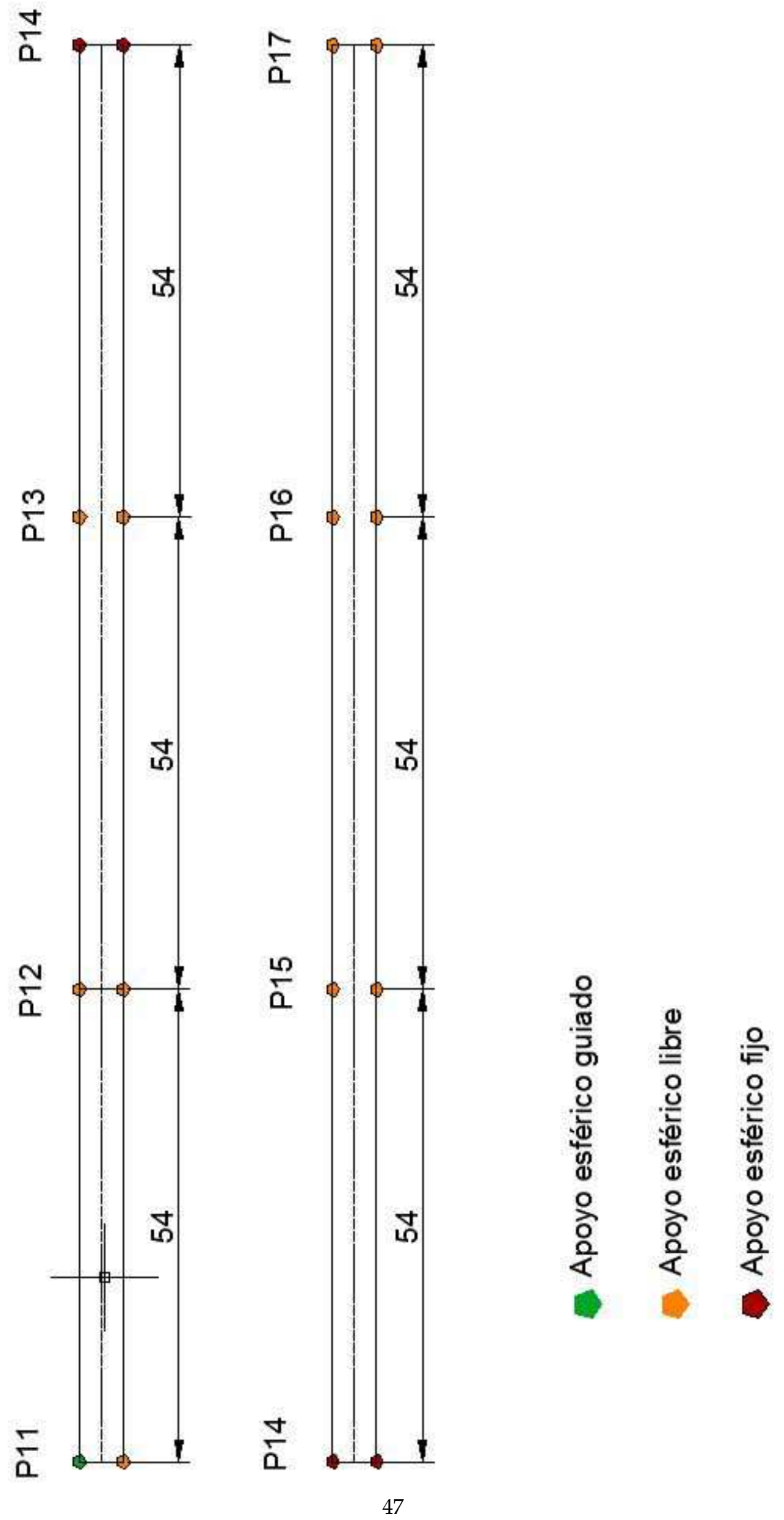

47

Figura 4.11: Vista en planta de la losa inferior. Situación y dirección de los apoyos de los vanos 12-17 sobre el arco. 


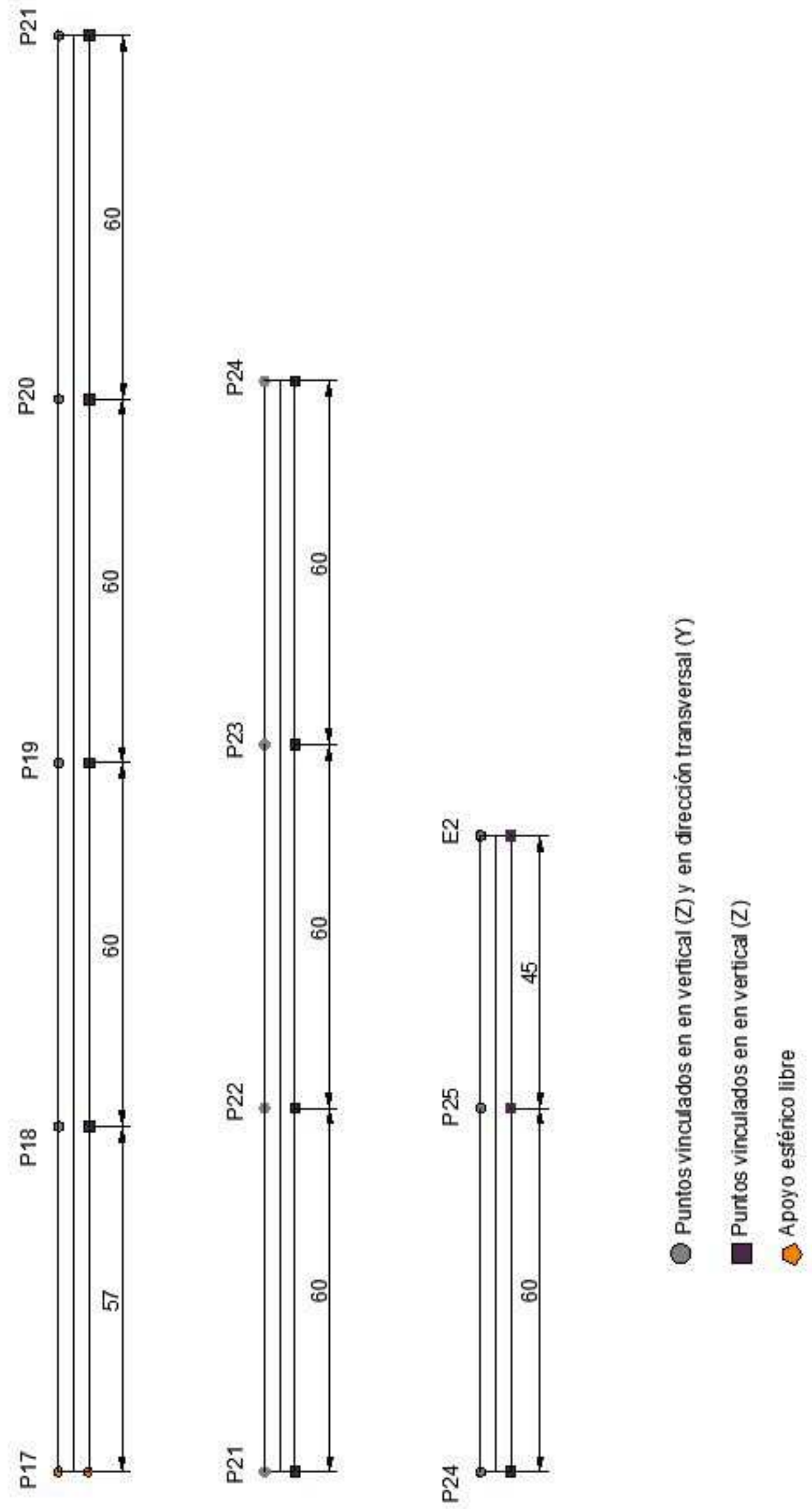

Figura 4.12: Vista en planta de la losa inferior. Situación y dirección de los apoyos de los vanos 18-26. 


\subsection{Aciones.}

El cálculo dinámico se realiza según se indica en el Apéndice B de la IAPF-07, considerándose los diez trenes definidos en el apéndice C.1 de dicha instrucción, los cuales reciben la denominación conjunta de Tren Dinámico Universal-A.

Siendo la velocidad de proyecto de la línea de $350 \mathrm{Km} / \mathrm{h}$, se han considerado velocidades de paso para dichos trenes desde $20 \mathrm{Km} / \mathrm{h}$ hasta $1.2 \times 350=420 \mathrm{~km} / \mathrm{h}$, en incrementos de $1 \mathrm{Km} / \mathrm{h}$.

El cálculo por superposición modal se realiza haciendo transitar las cargas a lo largo de una única línea de nudos situada en el eje de la vía. La posición de dicha línea sobre la losa superior se encuentra en la intersección del plano medio de la losa y el plano de simetría de las traviesas.

\subsection{Resultados a obtener y comprobaciones a relaizar.}

De acuerdo con la IAPF-07, del cálculo dinámico deben obtenerse los resultados necesarios para realizar las comprobaciones siguientes:

- Flecha dinámica máxima sobre vía sin irregularidades, con objeto de determinar el coeficiente de impacto.

- Flecha dinámica máxima a efectos de comprobación de ELS.

- Aceleración vertical máxima (ELS).

- Desplazamientos angulares máximos en los apoyos (ELS).

Los límites de cada tipo de respuesta asociados a los ELS son los siguientes:

- Flecha dinámica máxima : $7.5 \mathrm{~cm}$ para vanos de $45 \mathrm{~m}, 10 \mathrm{~cm}$ para vanos de $60 \mathrm{~m}, 9.5 \mathrm{~cm}$ para vanos de $57 \mathrm{~m}$ y $9 \mathrm{~cm}$ para vanos de $54 \mathrm{~m}$.

- Aceleración vertical máxima: $3.5 \mathrm{~m} / \mathrm{s}^{2}$

- Desplazamientos angulares máximos en los apoyos (ELS): $3.5 \cdot 10^{-3} \mathrm{rad}$ (en estribos).

Puntos para cálculo de resultados

Los puntos en los que se calculan las magnitudes descritas se muestra en la figura 4.13

Las aceleraciones verticales se calculan, para los seis vanos situados sobre el arco, en las secciones de centro y cuartos de vano y para cada sección en tres puntos: eje longitudinal del tablero y bordes de la banqueta de balasto (muretes). Por lo tanto obtenemos 54 puntos de postproceso para aceleraciones, denominados P1 a P54.

Para los vanos fuera del arco, se han tomado puntos de postproceso en el borde derecho de la banqueta de balasto para las secciones de centro de vano, obteniendose 20 puntos de postproceso denominados P73 a P92.

Las flechas se calculan en los vanos sobre el arco en el eje de la vía, en las secciones de centro y cuartos de vano. Ello da un total de 18 puntos denominados P55 a P72.

En la figura 4.13 se define la posición de los puntos de postproceso para los vanos sobre el arco. 

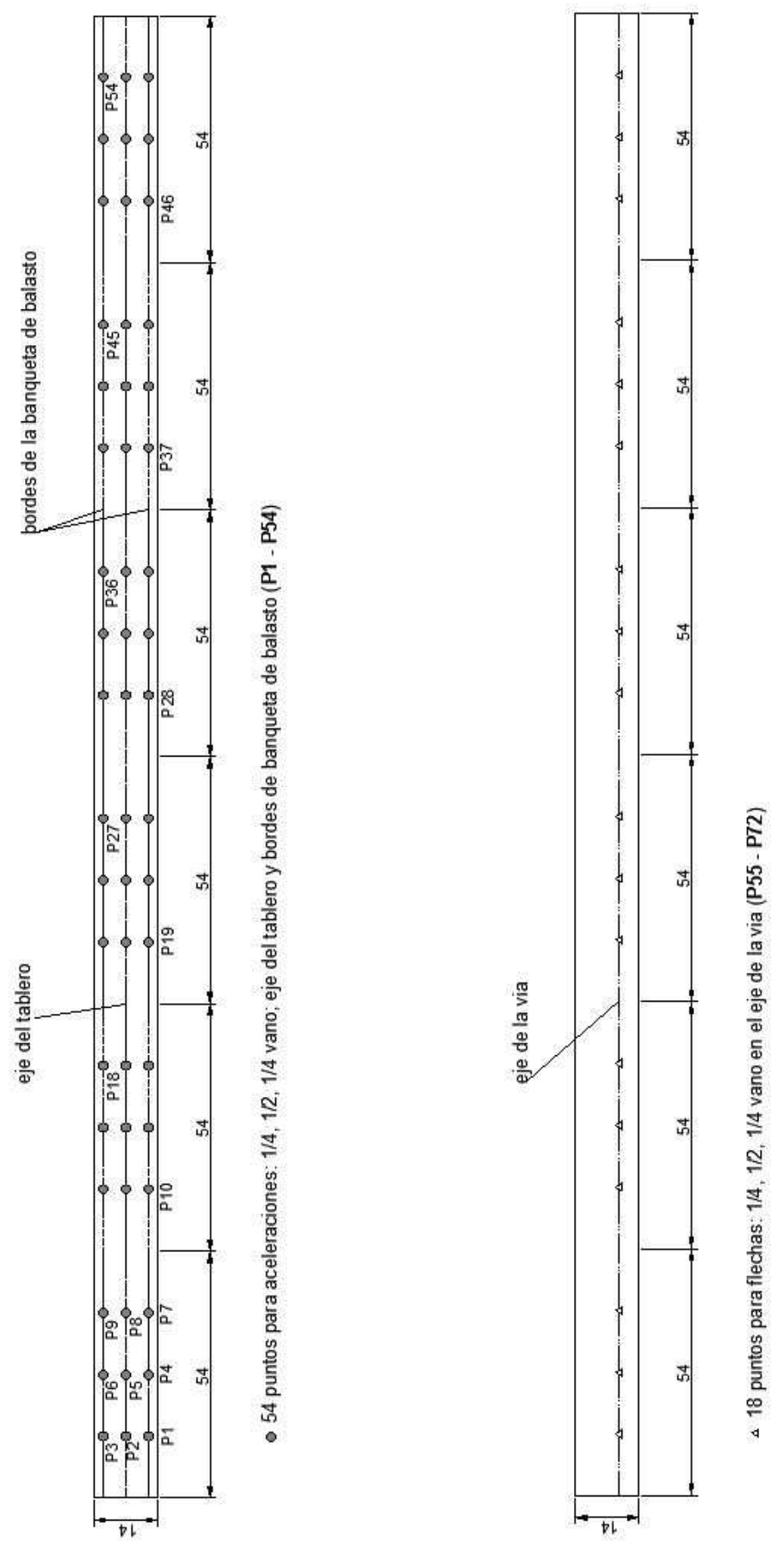

Figura 4.13: Puntos para cálculo de resultados. Vanos sobre el arco. 


\subsection{Resultados.}

\subsubsection{Frecuencias propias y modos de vibración}

El cálculo modal por elementos finitos del modelo descrito en el apartado 4.3 produce como resultado, para el valor superior de balasto, 688 modos con frecuencias de vibración inferiores a 30 Hz. Las frecuencias de dichos modos se recogen en la tabla 4.1-4.6.

Los seis primeros, de frecuencias de 0.5635 a $1.816 \mathrm{~Hz}$ corresponden a modos de flexión longitudinal del arco. A partir de dicho valor aparecen modos de flexión longitudinal del tablero, así como modos de torsión y flexión lateral acopladas en mayor o menor medida, y una gran cantidad de modos en los que predominan la flexión de los voladizos con diferente número de ondas. Las figuras $4.14,4.15,4.16,4.17,4.18$ y 4.21 muestran los modos que resultan representativos.

\begin{tabular}{|c|c|c|c|c|c|}
\hline Modo & Frecuencia(Hz) & Modo & Frecuencia $(\mathrm{Hz})$ & Modo & Frecuencia $(\mathrm{Hz})$ \\
\hline 1 & 0.56358 & 33 & 3.7634 & 65 & 6.3589 \\
\hline 2 & 0.68771 & 34 & 3.8397 & 66 & 6.3608 \\
\hline 3 & 1.1718 & 35 & 3.9103 & 67 & 6.3612 \\
\hline 4 & 1.6731 & 36 & 3.9461 & 68 & 6.375 \\
\hline 5 & 1.7731 & 37 & 4.1824 & 69 & 6.398 \\
\hline 6 & 1.8161 & 38 & 4.2806 & 70 & 6.4023 \\
\hline 7 & 2.0154 & 39 & 4.2873 & 71 & 6.4107 \\
\hline 8 & 2.0332 & 40 & 4.315 & 72 & 6.4461 \\
\hline 9 & 2.0515 & 41 & 4.4213 & 73 & 6.4478 \\
\hline 10 & 2.1029 & 42 & 4.8248 & 74 & 6.4505 \\
\hline 11 & 2.1217 & 43 & 5.0662 & 75 & 6.4547 \\
\hline 12 & 2.1217 & 44 & 5.3499 & 76 & 6.4559 \\
\hline 13 & 2.125 & 45 & 6.0736 & 77 & 6.462 \\
\hline 14 & 2.1605 & 46 & 6.1468 & 78 & 6.4789 \\
\hline 15 & 2.193 & 47 & 6.159 & 79 & 6.5038 \\
\hline 16 & 2.2951 & 48 & 6.2174 & 80 & 6.51 \\
\hline 18 & 2.4891 & 50 & 6.3076 & 82 & 6.5421 \\
\hline 19 & 2.5921 & 51 & 6.3299 & 83 & 6.5886 \\
\hline 20 & 2.7094 & 52 & 6.3304 & 84 & 6.6148 \\
\hline 21 & 2.7839 & 53 & 6.3323 & 85 & 6.6352 \\
\hline 22 & 2.9149 & 54 & 6.3339 & 86 & 6.6352 \\
\hline 23 & 2.9827 & 55 & 6.3359 & 87 & 6.6455 \\
\hline 24 & 3.0397 & 56 & 6.3384 & 88 & 6.6793 \\
\hline 25 & 3.112 & 57 & 6.3392 & 89 & 6.6968 \\
\hline 26 & 3.2621 & 58 & 6.3405 & 90 & 6.7277 \\
\hline 27 & 3.3501 & 59 & 6.3454 & 91 & 6.7486 \\
\hline 28 & 3.4467 & 60 & 6.3455 & 92 & 6.7636 \\
\hline 29 & 3.4838 & 61 & 6.3506 & 93 & 6.7813 \\
\hline 30 & 3.5297 & 62 & 6.3517 & 94 & 6.7849 \\
\hline 31 & 3.5956 & 63 & 6.3552 & 95 & 6.9513 \\
\hline 32 & 3.678 & 64 & 6.3571 & 96 & 7.0115 \\
\hline
\end{tabular}

Cuadro 4.1: Frecuencias propias 


\begin{tabular}{|c|c|c|c|c|c|}
\hline Modo & Frecuencia(Hz) & Modo & Frecuencia $(\mathrm{Hz})$ & Modo & Frecuencia (Hz) \\
\hline 97 & 7.0124 & 141 & 7.3925 & 185 & 7.9218 \\
\hline 99 & 7.0165 & 143 & 7.406 & 187 & 7.9511 \\
\hline 100 & 7.02 & 144 & 7.4271 & 188 & 7.9815 \\
\hline 101 & 7.0247 & 145 & 7.433 & 189 & 7.9822 \\
\hline 102 & 7.0322 & 146 & 7.4383 & 190 & 8.0027 \\
\hline 103 & 7.0359 & 147 & 7.449 & 191 & 8.0086 \\
\hline 104 & 7.0483 & 148 & 7.4558 & 192 & 8.01 \\
\hline 105 & 7.0496 & 149 & 7.468 & 193 & 8.0445 \\
\hline 106 & 7.0651 & 150 & 7.4733 & 194 & 8.0501 \\
\hline 107 & 7.066 & 151 & 7.4893 & 195 & 8.0726 \\
\hline 108 & 7.0767 & 152 & 7.4896 & 196 & 8.0811 \\
\hline 109 & 7.0805 & 153 & 7.51 & 197 & 8.1006 \\
\hline 110 & 7.0859 & 154 & 7.5144 & 198 & 8.1074 \\
\hline 111 & 7.0932 & 155 & 7.5297 & 199 & 8.1184 \\
\hline 112 & 7.0999 & 156 & 7.5376 & 200 & 8.1387 \\
\hline 113 & 7.106 & 157 & 7.5499 & 201 & 8.1558 \\
\hline 114 & 7.1135 & 158 & 7.5714 & 202 & 8.1727 \\
\hline 115 & 7.1168 & 159 & 7.5766 & 203 & 8.1854 \\
\hline 116 & 7.1229 & 160 & 7.5906 & 204 & 8.1873 \\
\hline 117 & 7.1232 & 161 & 7.5928 & 205 & 8.1888 \\
\hline 118 & 7.1452 & 162 & 7.6152 & 206 & 8.1976 \\
\hline 119 & 7.1472 & 163 & 7.6369 & 207 & 8.2055 \\
\hline 120 & 7.1473 & 164 & 7.6377 & 208 & 8.2072 \\
\hline 121 & 7.1798 & 165 & 7.654 & 209 & 8.2168 \\
\hline 122 & 7.2168 & 166 & 7.6605 & 210 & 8.2247 \\
\hline 123 & 7.2518 & 167 & 7.6846 & 211 & 8.2339 \\
\hline 124 & 7.2631 & 168 & 7.7081 & 212 & 8.241 \\
\hline 125 & 7.2744 & 169 & 7.7161 & 213 & 8.2445 \\
\hline 126 & 7.2744 & 170 & 7.7197 & 214 & 8.2609 \\
\hline 127 & 7.2872 & 171 & 7.7297 & 215 & 8.2623 \\
\hline 128 & 7.2889 & 172 & 7.7516 & 216 & 8.2746 \\
\hline 129 & 7.2954 & 173 & 7.7652 & 217 & 8.2776 \\
\hline 130 & 7.2986 & 174 & 7.7691 & 218 & 8.2876 \\
\hline 131 & 7.3092 & 175 & 7.7924 & 219 & 8.2974 \\
\hline 132 & 7.3132 & 176 & 7.8009 & 220 & 8.3027 \\
\hline 133 & 7.3286 & 177 & 7.8126 & 221 & 8.313 \\
\hline 134 & 7.3355 & 178 & 7.8139 & 222 & 8.3232 \\
\hline 135 & 7.3539 & 179 & 7.8353 & 223 & 8.333 \\
\hline 136 & 7.3563 & 180 & 7.8419 & 224 & 8.3394 \\
\hline 137 & 7.3583 & 181 & 7.8687 & 225 & 8.3499 \\
\hline 138 & 7.3659 & 182 & 7.8751 & 226 & 8.3662 \\
\hline 139 & 7.376 & 183 & 7.8932 & 227 & 8.3739 \\
\hline 140 & 7.3896 & 184 & 7.9097 & 228 & 8.3873 \\
\hline
\end{tabular}

Cuadro 4.2: Frecuencias propias (continuación) 


\begin{tabular}{|c|c|c|c|c|c|}
\hline Modo & Frecuencia(Hz) & Modo & Frecuencia (Hz) & Modo & Frecuencia $(\mathrm{Hz})$ \\
\hline 229 & 8.388 & 273 & 9.1255 & 316 & 10.251 \\
\hline 230 & 8.397 & 274 & 9.1403 & 317 & 10.266 \\
\hline 231 & 8.4152 & 275 & 9.183 & 318 & 10.322 \\
\hline 232 & 8.4256 & 276 & 9.193 & 319 & 10.339 \\
\hline 233 & 8.4266 & 277 & 9.2283 & 320 & 10.349 \\
\hline 234 & 8.4421 & 278 & 9.2401 & 321 & 10.397 \\
\hline 235 & 8.4473 & 279 & 9.2468 & 322 & 10.414 \\
\hline 236 & 8.4638 & 280 & 9.2925 & 323 & 10.443 \\
\hline 237 & 8.4648 & 281 & 9.3039 & 324 & 10.455 \\
\hline 238 & 8.4731 & 282 & 9.3478 & 325 & 10.473 \\
\hline 239 & 8.4864 & 283 & 9.3586 & 326 & 10.491 \\
\hline 240 & 8.5027 & 284 & 9.4057 & 327 & 10.549 \\
\hline 241 & 8.5032 & 285 & 9.4174 & 328 & 10.561 \\
\hline 242 & 8.5255 & 286 & 9.4653 & 329 & 10.57 \\
\hline 243 & 8.5434 & 287 & 9.4764 & 330 & 10.63 \\
\hline 244 & 8.5661 & 288 & 9.5242 & 331 & 10.65 \\
\hline 245 & 8.5832 & 289 & 9.5357 & 332 & 10.672 \\
\hline 246 & 8.5842 & 290 & 9.5855 & 333 & 10.711 \\
\hline 247 & 8.6276 & 291 & 9.5971 & 334 & 10.732 \\
\hline 248 & 8.6283 & 292 & 9.6475 & 335 & 10.789 \\
\hline 249 & 8.6602 & 293 & 9.6593 & 336 & 10.804 \\
\hline 250 & 8.6696 & 294 & 9.7105 & 337 & 10.817 \\
\hline 251 & 8.6784 & 295 & 9.7228 & 338 & 10.849 \\
\hline 252 & 8.6932 & 296 & 9.75 & 339 & 10.886 \\
\hline 253 & 8.7052 & 297 & 9.775 & 340 & 10.903 \\
\hline 254 & 8.7102 & 298 & 9.7856 & 341 & 10.97 \\
\hline 255 & 8.7129 & 299 & 9.7994 & 342 & 10.976 \\
\hline 256 & 8.7475 & 300 & 9.8399 & 343 & 10.989 \\
\hline 257 & 8.7549 & 301 & 9.8528 & 344 & 11.049 \\
\hline 258 & 8.7872 & 302 & 9.9031 & 345 & 11.082 \\
\hline 259 & 8.7992 & 303 & 9.9178 & 346 & 11.127 \\
\hline 260 & 8.8314 & 304 & 9.9728 & 347 & 11.137 \\
\hline 261 & 8.8446 & 305 & 9.9847 & 348 & 11.163 \\
\hline 262 & 8.8773 & 306 & 10.045 & 349 & 11.172 \\
\hline 263 & 8.8912 & 307 & 10.055 & 350 & 11.236 \\
\hline 264 & 8.9239 & 308 & 10.09 & 351 & 11.246 \\
\hline 265 & 8.9387 & 309 & 10.109 & 352 & 11.265 \\
\hline 266 & 8.9732 & 310 & 10.122 & 353 & 11.333 \\
\hline 267 & 8.9874 & 311 & 10.126 & 354 & 11.363 \\
\hline 268 & 8.9936 & 312 & 10.18 & 355 & 11.385 \\
\hline 269 & 9.0249 & 313 & 10.188 & 356 & 11.425 \\
\hline 270 & 9.0371 & 314 & 10.194 & 357 & 11.458 \\
\hline 271 & 9.0774 & 315 & 10.241 & 358 & 11.464 \\
\hline 272 & 9.0877 & 316 & 10.251 & 359 & 11.523 \\
\hline
\end{tabular}

Cuadro 4.3: Frecuencias propias (continuación) 


\begin{tabular}{|c|c|c|c|c|c|}
\hline Modo & Frecuencia(Hz) & Modo & Frecuencia $(\mathrm{Hz})$ & Modo & Frecuencia $(\mathrm{Hz})$ \\
\hline 360 & 11.557 & 404 & 13.249 & 448 & 14.3 \\
\hline 361 & 11.567 & 405 & 13.253 & 449 & 14.367 \\
\hline 362 & 11.567 & 406 & 13.308 & 450 & 14.415 \\
\hline 363 & 11.62 & 407 & 13.335 & 451 & 14.449 \\
\hline 364 & 11.633 & 408 & 13.35 & 452 & 14.57 \\
\hline 365 & 11.657 & 409 & 13.353 & 453 & 14.6 \\
\hline 366 & 11.678 & 410 & 13.365 & 454 & 14.629 \\
\hline 367 & 11.722 & 411 & 13.373 & 455 & 14.726 \\
\hline 368 & 11.752 & 412 & 13.379 & 456 & 14.759 \\
\hline 369 & 11.76 & 413 & 13.39 & 457 & 14.887 \\
\hline 370 & 11.821 & 414 & 13.409 & 458 & 14.918 \\
\hline 371 & 11.861 & 415 & 13.411 & 459 & 15.027 \\
\hline 372 & 11.915 & 416 & 13.42 & 460 & 15.051 \\
\hline 373 & 11.924 & 417 & 13.44 & 461 & 15.076 \\
\hline 374 & 11.967 & 418 & 13.45 & 462 & 15.162 \\
\hline 375 & 12.029 & 419 & 13.457 & 463 & 15.209 \\
\hline 376 & 12.049 & 420 & 13.485 & 464 & 15.227 \\
\hline 377 & 12.075 & 421 & 13.493 & 465 & 15.236 \\
\hline 378 & 12.104 & 422 & 13.522 & 466 & 15.256 \\
\hline 379 & 12.142 & 423 & 13.548 & 467 & 15.264 \\
\hline 380 & 12.163 & 424 & 13.559 & 468 & 15.276 \\
\hline 381 & 12.178 & 425 & 13.564 & 469 & 15.383 \\
\hline 382 & 12.248 & 426 & 13.578 & 470 & 15.407 \\
\hline 383 & 12.291 & 427 & 13.579 & 471 & 15.558 \\
\hline 384 & 12.304 & 428 & 13.582 & 472 & 15.565 \\
\hline 385 & 12.348 & 429 & 13.675 & 473 & 15.66 \\
\hline 386 & 12.352 & 430 & 13.713 & 474 & 15.723 \\
\hline 387 & 12.4 & 431 & 13.714 & 475 & 15.734 \\
\hline 388 & 12.42 & 432 & 13.727 & 476 & 15.903 \\
\hline 389 & 12.46 & 433 & 13.796 & 477 & 15.911 \\
\hline 390 & 12.514 & 434 & 13.811 & 478 & 15.961 \\
\hline 391 & 12.576 & 435 & 13.857 & 479 & 16.086 \\
\hline 392 & 12.589 & 436 & 13.893 & 480 & 16.089 \\
\hline 393 & 12.631 & 437 & 13.919 & 481 & 16.269 \\
\hline 394 & 12.689 & 438 & 13.942 & 482 & 16.273 \\
\hline 395 & 12.748 & 439 & 13.954 & 483 & 16.458 \\
\hline 396 & 12.759 & 440 & 14 & 484 & 16.464 \\
\hline 397 & 12.808 & 441 & 14.004 & 485 & 16.65 \\
\hline 398 & 12.869 & 442 & 14.047 & 486 & 16.659 \\
\hline 399 & 12.928 & 443 & 14.076 & 487 & 16.846 \\
\hline 400 & 12.993 & 444 & 14.115 & 488 & 16.85 \\
\hline 401 & 13.052 & 445 & 14.129 & 489 & 16.861 \\
\hline 402 & 13.12 & 446 & 14.144 & 490 & 17.048 \\
\hline 403 & 13.179 & 447 & 14.263 & 491 & 17.068 \\
\hline
\end{tabular}

Cuadro 4.4: Frecuencias propias (continuación) 


\begin{tabular}{|c|c|c|c|c|c|}
\hline Modo & Frecuencia(Hz) & Modo & Frecuencia $(\mathrm{Hz})$ & Modo & Frecuencia $(\mathrm{Hz})$ \\
\hline 492 & 17.208 & 535 & 19.425 & 578 & 21.375 \\
\hline 493 & 17.252 & 536 & 19.478 & 579 & 21.39 \\
\hline 494 & 17.264 & 537 & 19.495 & 580 & 21.52 \\
\hline 495 & 17.281 & 538 & 19.518 & 581 & 21.567 \\
\hline 496 & 17.312 & 539 & 19.568 & 582 & 21.686 \\
\hline 497 & 17.455 & 540 & 19.594 & 583 & 21.731 \\
\hline 498 & 17.465 & 541 & 19.595 & 584 & 21.753 \\
\hline 499 & 17.498 & 542 & 19.658 & 585 & 21.855 \\
\hline 500 & 17.506 & 543 & 19.667 & 586 & 22.031 \\
\hline 501 & 17.634 & 544 & 19.681 & 587 & 22.171 \\
\hline 502 & 17.686 & 545 & 19.698 & 588 & 22.281 \\
\hline 503 & 17.699 & 546 & 19.724 & 589 & 22.312 \\
\hline 504 & 17.721 & 547 & 19.759 & 590 & 22.331 \\
\hline 505 & 17.815 & 548 & 19.791 & 591 & 22.484 \\
\hline 506 & 17.917 & 549 & 19.797 & 592 & 22.621 \\
\hline 507 & 17.944 & 550 & 19.823 & 593 & 22.674 \\
\hline 508 & 17.997 & 551 & 19.848 & 594 & 22.813 \\
\hline 509 & 18.082 & 552 & 19.856 & 595 & 22.894 \\
\hline 510 & 18.131 & 553 & 19.865 & 596 & 22.947 \\
\hline 511 & 18.183 & 554 & 19.873 & 597 & 23.142 \\
\hline 512 & 18.203 & 555 & 19.903 & 598 & 23.268 \\
\hline 513 & 18.362 & 556 & 20.001 & 599 & 23.36 \\
\hline 514 & 18.413 & 557 & 20.062 & 600 & 23.484 \\
\hline 515 & 18.502 & 558 & 20.08 & 601 & 23.587 \\
\hline 516 & 18.578 & 559 & 20.167 & 602 & 23.608 \\
\hline 517 & 18.591 & 560 & 20.308 & 603 & 23.832 \\
\hline 518 & 18.621 & 561 & 20.328 & 604 & 23.945 \\
\hline 519 & 18.649 & 562 & 20.367 & 605 & 24.187 \\
\hline 520 & 18.824 & 563 & 20.409 & 606 & 24.295 \\
\hline 521 & 18.903 & 564 & 20.441 & 607 & 24.547 \\
\hline 522 & 19 & 565 & 20.58 & 608 & 24.637 \\
\hline 523 & 19.062 & 566 & 20.605 & 609 & 24.717 \\
\hline 524 & 19.09 & 567 & 20.691 & 610 & 24.906 \\
\hline 525 & 19.132 & 568 & 20.695 & 611 & 24.918 \\
\hline 526 & 19.168 & 569 & 20.843 & 612 & 24.928 \\
\hline 527 & 19.292 & 570 & 20.967 & 613 & 24.998 \\
\hline 528 & 19.333 & 571 & 21.117 & 614 & 25.207 \\
\hline 529 & 19.34 & 572 & 21.123 & 615 & 25.279 \\
\hline 530 & 19.369 & 573 & 21.158 & 616 & 25.36 \\
\hline 531 & 19.374 & 574 & 21.208 & 617 & 25.575 \\
\hline 532 & 19.375 & 575 & 21.232 & 618 & 25.658 \\
\hline 533 & 19.391 & 576 & 21.28 & 619 & 25.682 \\
\hline 534 & 19.396 & 577 & 21.349 & 620 & 25.727 \\
\hline & & & & & \\
\hline
\end{tabular}

Cuadro 4.5: Frecuencias propias (continuación) 


\begin{tabular}{|c|c|c|c|}
\hline Modo & Frecuencia(Hz) & Modo & Frecuencia $(\mathrm{Hz})$ \\
\hline 621 & 25.737 & 655 & 27.601 \\
\hline 622 & 25.934 & 656 & 27.645 \\
\hline 623 & 25.98 & 657 & 27.669 \\
\hline 624 & 26.036 & 658 & 27.684 \\
\hline 625 & 26.08 & 659 & 27.716 \\
\hline 626 & 26.134 & 660 & 27.736 \\
\hline 627 & 26.265 & 661 & 27.777 \\
\hline 628 & 26.384 & 662 & 27.798 \\
\hline 629 & 26.393 & 663 & 27.815 \\
\hline 630 & 26.406 & 664 & 27.821 \\
\hline 631 & 26.626 & 665 & 27.856 \\
\hline 632 & 26.671 & 666 & 27.866 \\
\hline 633 & 26.696 & 667 & 27.875 \\
\hline 634 & 26.737 & 668 & 27.997 \\
\hline 635 & 26.8 & 669 & 27.998 \\
\hline 636 & 26.997 & 670 & 28.078 \\
\hline 637 & 27.07 & 671 & 28.37 \\
\hline 638 & 27.113 & 672 & 28.394 \\
\hline 639 & 27.151 & 673 & 28.43 \\
\hline 640 & 27.193 & 674 & 28.493 \\
\hline 641 & 27.202 & 675 & 28.626 \\
\hline 642 & 27.211 & 676 & 28.653 \\
\hline 643 & 27.242 & 677 & 28.769 \\
\hline 644 & 27.275 & 678 & 28.828 \\
\hline 645 & 27.315 & 679 & 28.889 \\
\hline 646 & 27.371 & 680 & 28.909 \\
\hline 647 & 27.406 & 681 & 29.17 \\
\hline 648 & 27.408 & 682 & 29.243 \\
\hline 649 & 27.45 & 683 & 29.302 \\
\hline 650 & 27.499 & 684 & 29.68 \\
\hline 651 & 27.53 & 685 & 29.776 \\
\hline 652 & 27.533 & 686 & 29.814 \\
\hline 653 & 27.536 & 687 & 29.953 \\
\hline 654 & 27.581 & 688 & 29.979 \\
\hline
\end{tabular}

Cuadro 4.6: Frecuencias propias (continuación) 


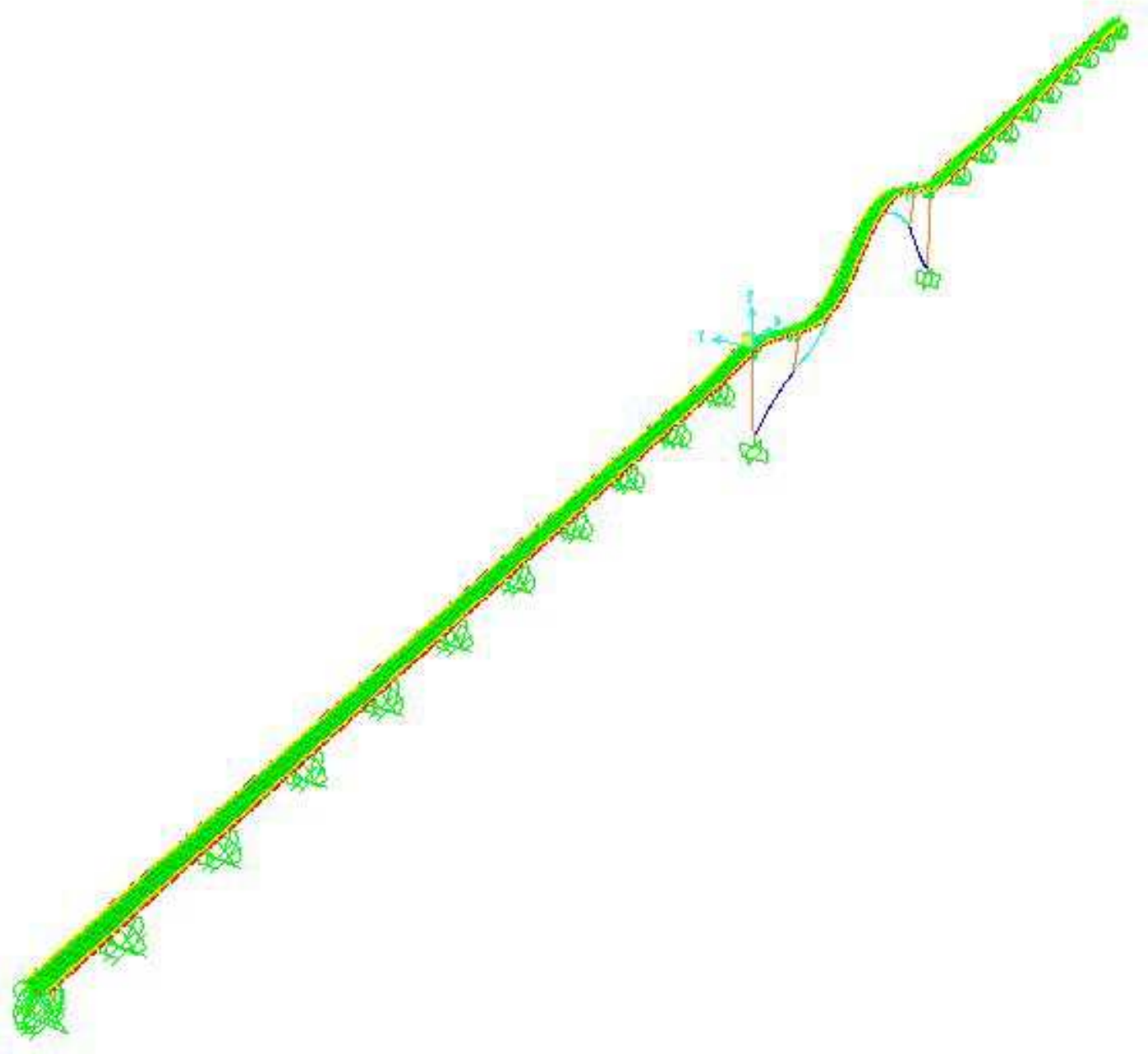

Figura 4.14: Primer modo de vibración, de flexión acoplada de arco $(0.5638 \mathrm{~Hz})$. 


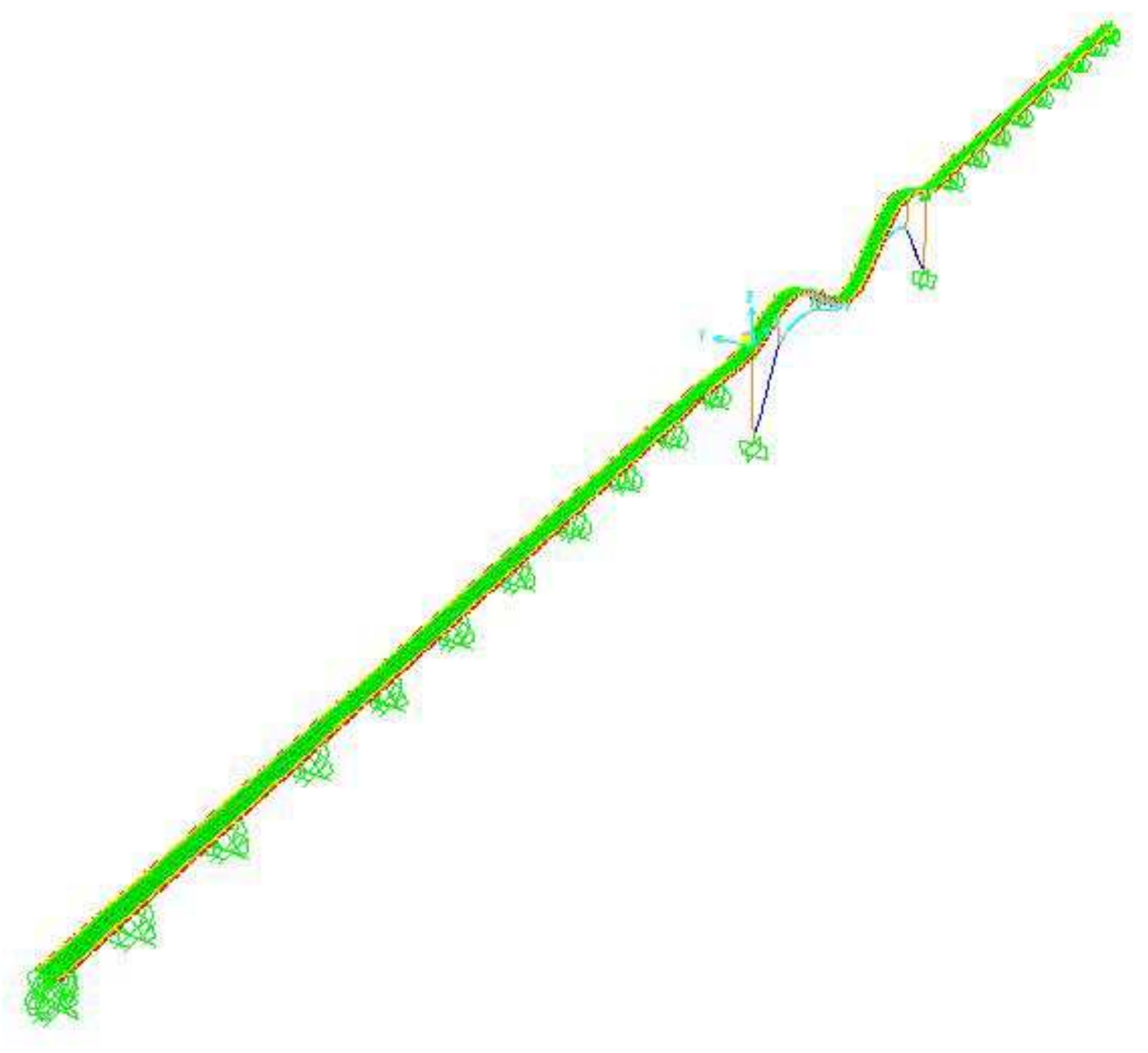

Figura 4.15: Segundo modo de vibración, de flexión acoplada de arco $(0.68771 \mathrm{~Hz})$. 


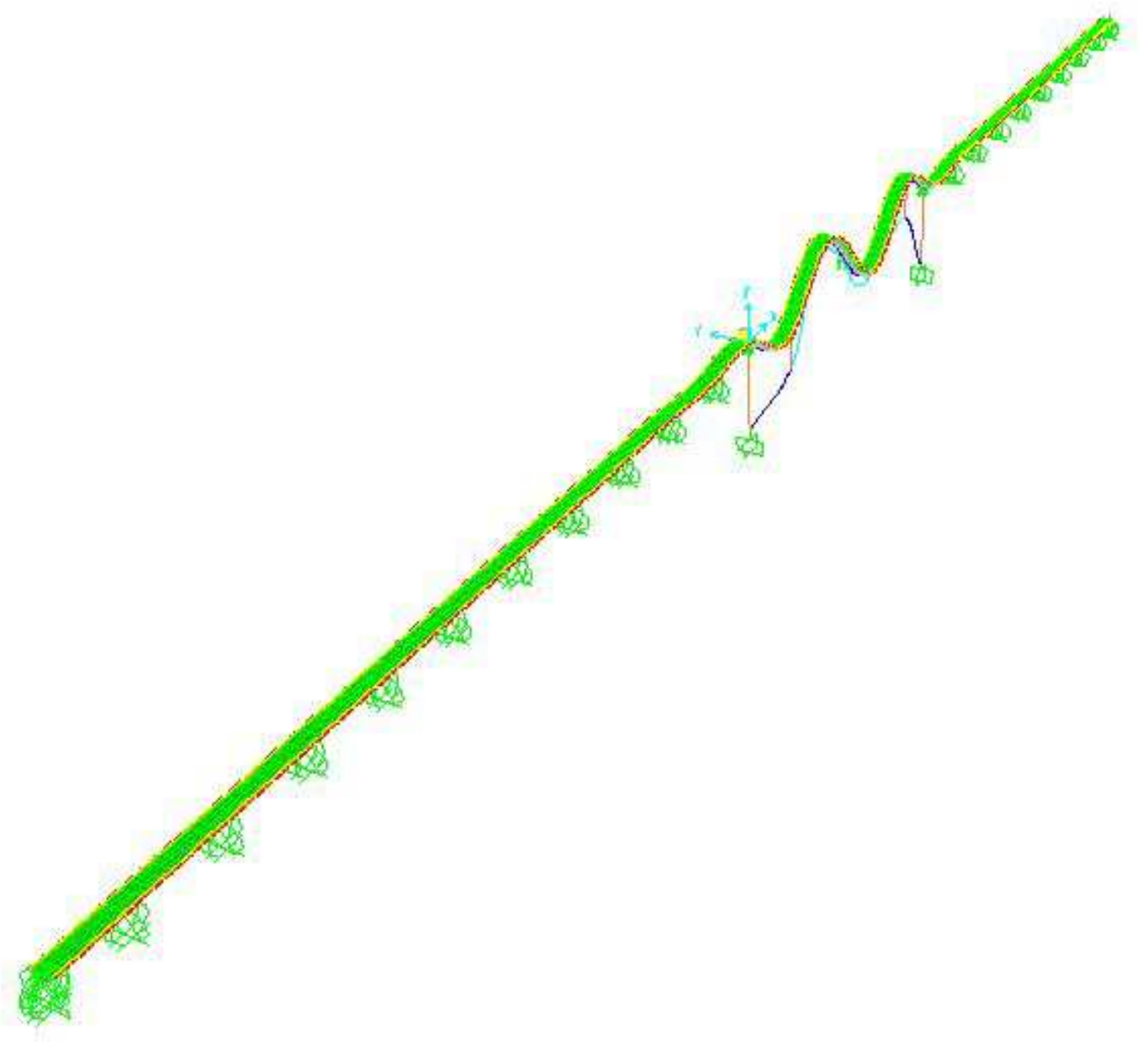

Figura 4.16: Tercer modo de vibración, de flexión acoplada de arco $(1.1718$ Hz). 


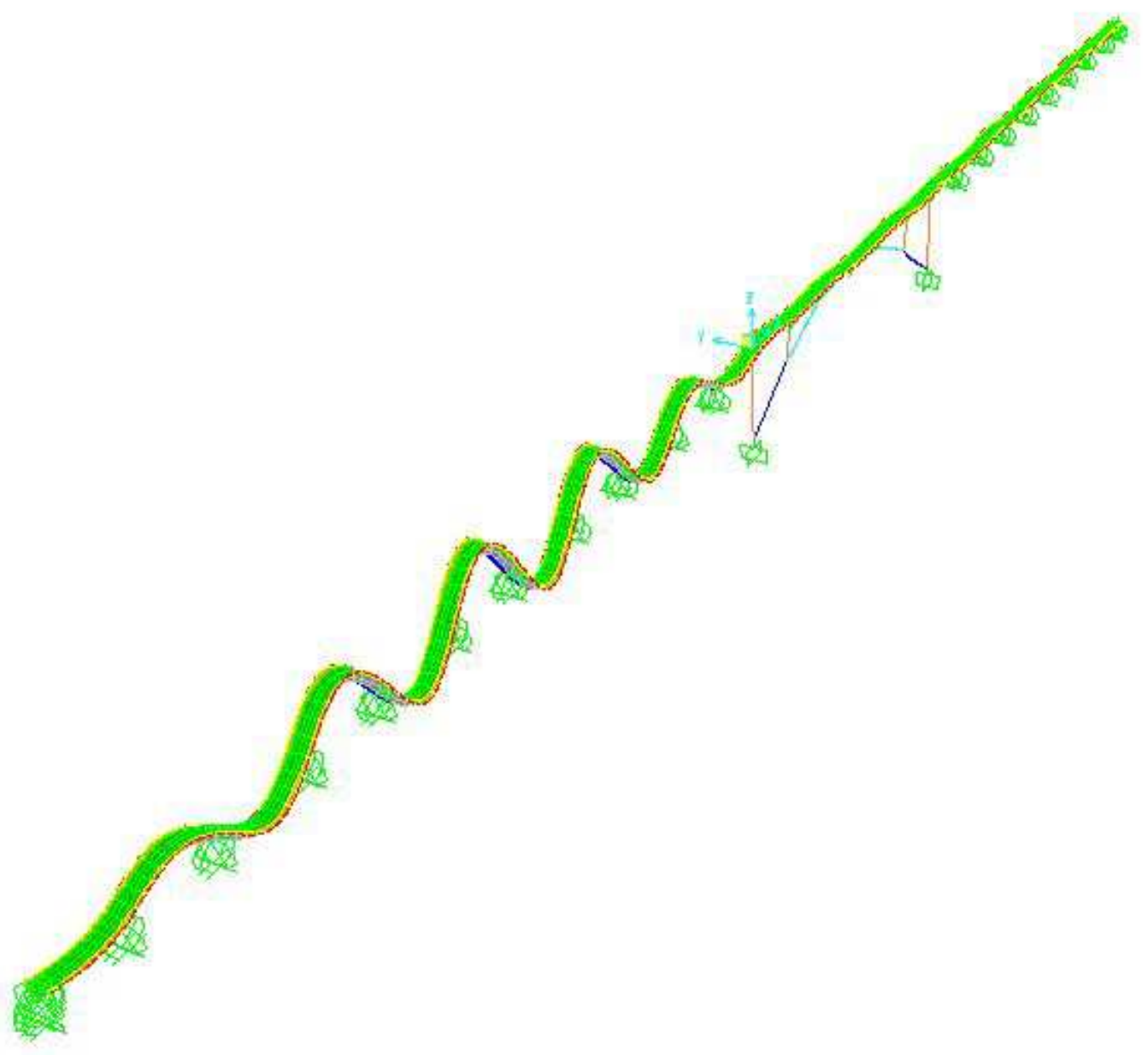

Figura 4.17: Modo de vibración n 7 (2.0154 Hz). 


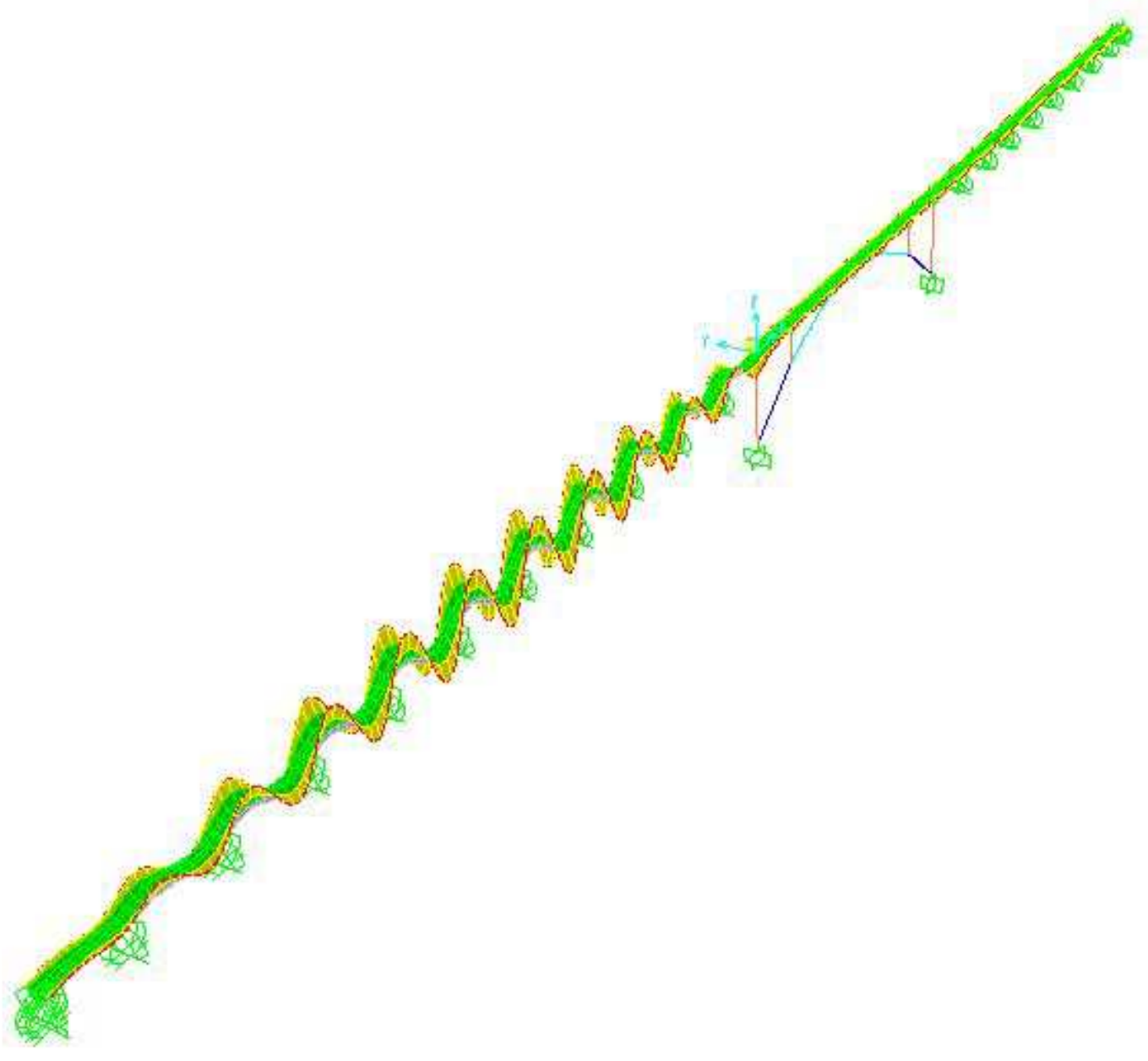

Figura 4.18: Modo de vibración no 46 (6.1468 Hz). 


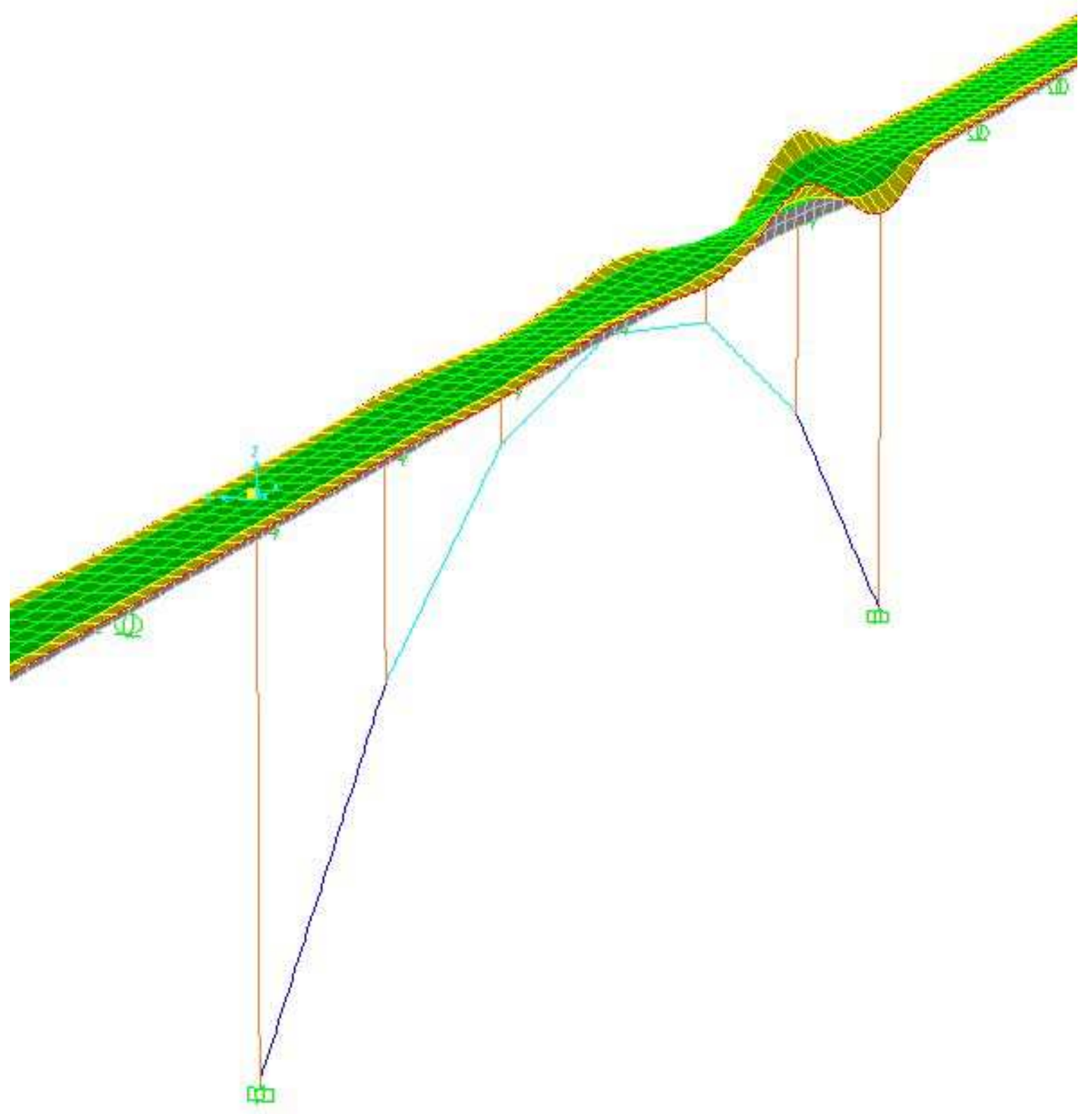

Figura 4.19: Modo de vibración n ${ }^{\circ}$ 72, torsión en vanos sobre el arco (6.4461Hz). 


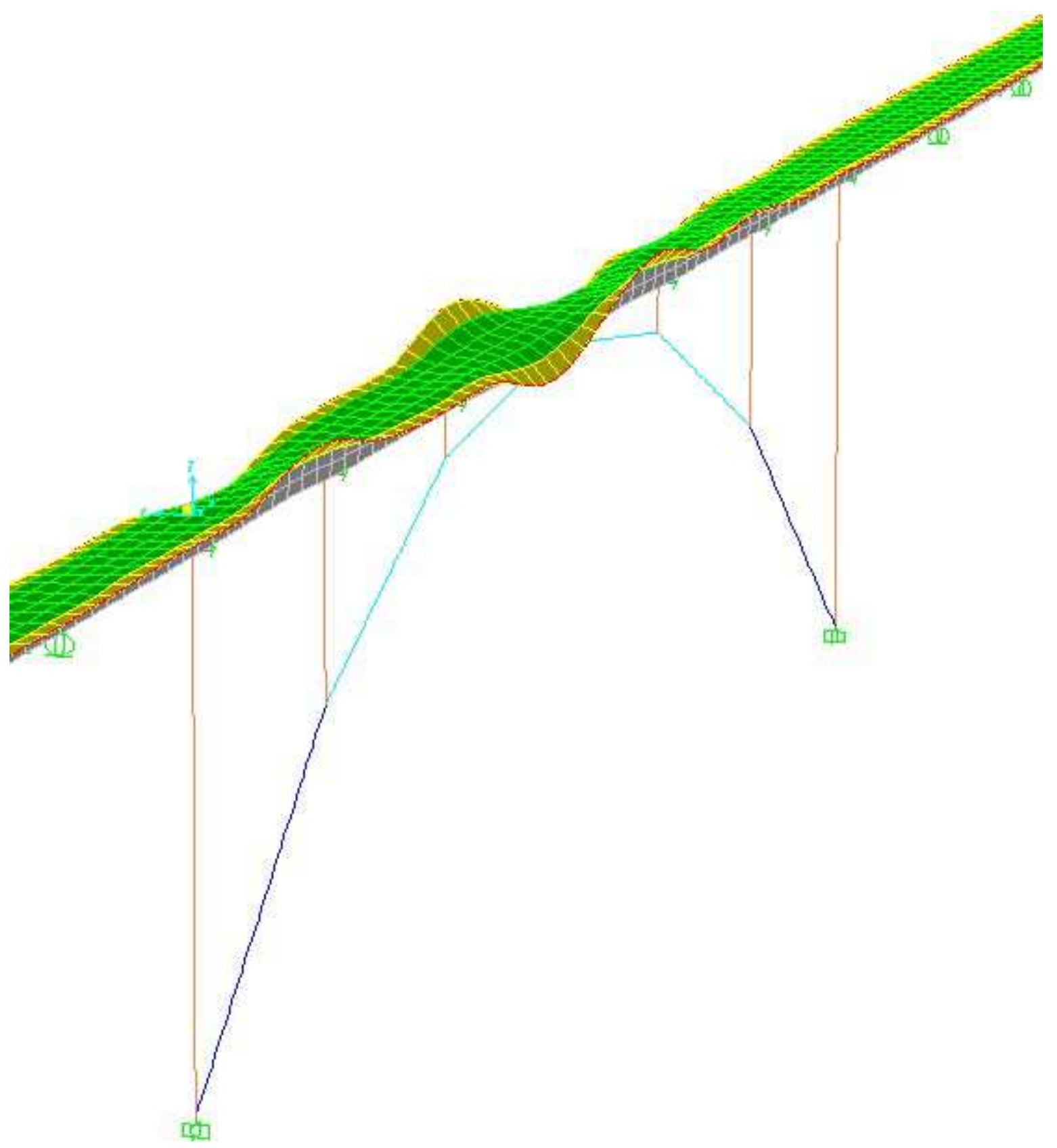

Figura 4.20: Modo de vibración n ${ }^{\circ} 75$, torsión en vanos sobre el arco $(6.4547 \mathrm{~Hz})$. 


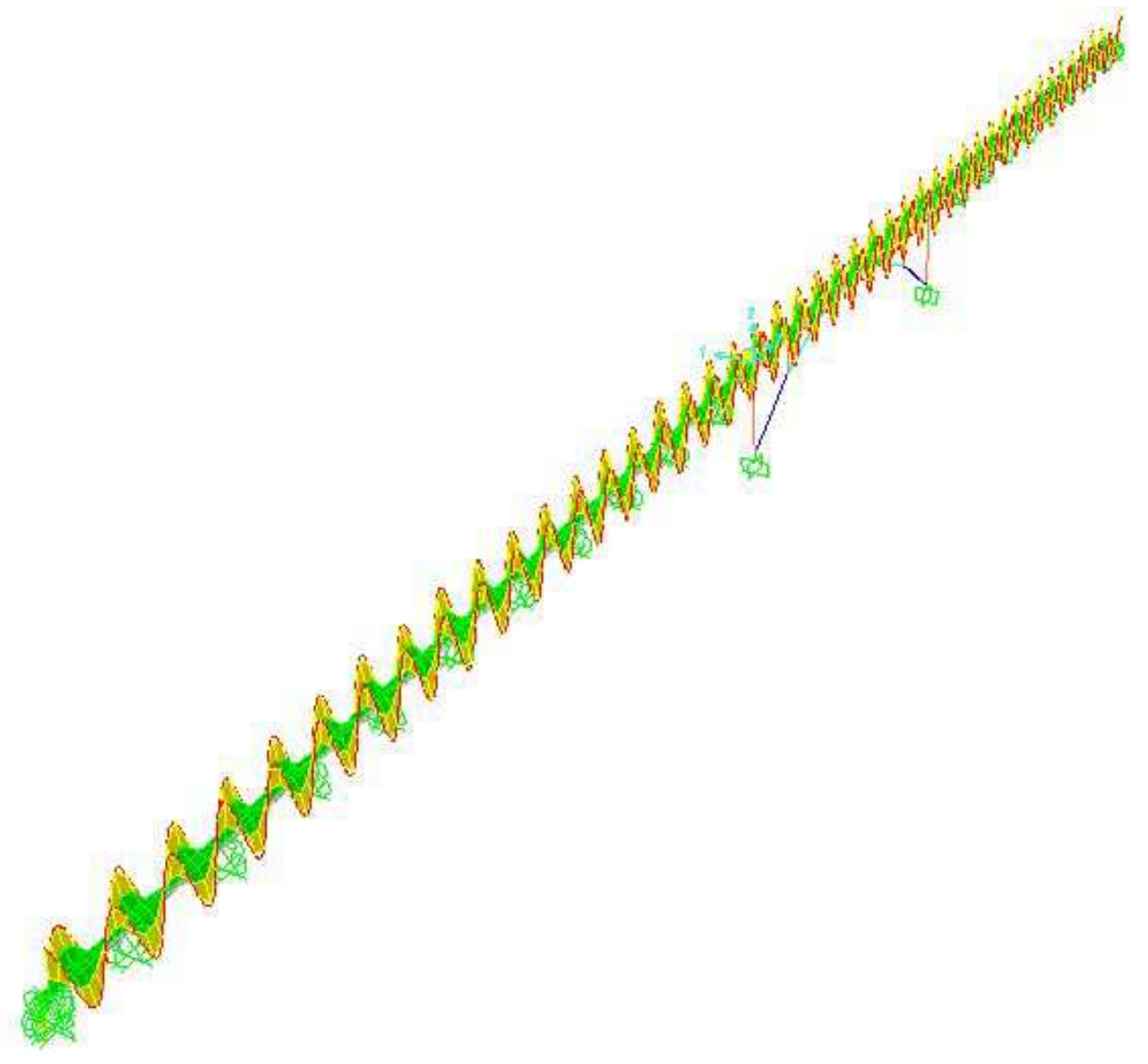

Figura 4.21: Modo de vibración no 208 (8.2072Hz). 


\subsubsection{Valores máximos y valores límite}

Para tener en cuenta los efectos de las irregularidades de la vía, la IAPF-07 establece, para la tipología y velocidad de proyecto del viaducto tratado, el cálculo del coeficiente $\varphi^{\prime \prime}$ para amplificar los resultados calculados. Para el viaducto sobre el río Tajo en el embalse de Alcántara, este coeficiente se ha evaluado y obtenemos un valor negativo por lo que tomanos $\varphi^{\prime \prime}=0$. Se ha considerado la expresión (B12) de la IAPF-07, con $L_{\Phi}=85,846 \mathrm{~m} ; \mathrm{f}_{0}=0,5 \mathrm{~Hz}$.

\subsubsection{Valores máximos. Tramo de arco.}

Aceleración

Máximo valor. $A_{v}^{\text {ideal }}=1,066 \mathrm{~m} / \mathrm{s}^{2}$

Punto: 33 (Situado en el vano 15).

Tren: A1.

Velocidad: $420 \mathrm{Km} / \mathrm{h}$.

$A_{v}=A_{v} \cdot\left(1+0,5 \varphi^{\prime \prime}\right)=1,066 \mathrm{~m} / \mathrm{s}^{2}$

Valor límite: $0.35 \mathrm{~g}=3.5 \mathrm{~m} / \mathrm{s}^{2}$.

Conclusión: No se supera el límite de aceleración máxima permitido por la IAPF-07.

\section{Desplazamiento}

Máximo valor: $\delta_{\text {din, } \text { real }}^{\text {deal }}=3,564 E^{-2} \mathrm{~m}$

Punto: 64 (primer cuarto de vano, vano 15,sobre el arco).

Tren: A2.

Velocidad: $420 \mathrm{Km} / \mathrm{h}$.

$\delta_{v}=\delta_{\text {din, real }} \cdot\left(1+0,5 \varphi^{\prime \prime}\right)=3,564 E^{-2} \mathrm{~m}$

Valores límite: $7.5 \mathrm{~cm}$ para vanos de $45 \mathrm{~m}, 10 \mathrm{~cm}$ para vanos de $60 \mathrm{~m}, 9.5 \mathrm{~cm}$ para vanos de $57 \mathrm{~m}$ y $9 \mathrm{~cm}$ para vanos de $54 \mathrm{~m}$.

Conclusión: No se supera el límite de desplazamiento máximo permitido por la IAPF-07. 
4.6.4. Valores máximos. Resto de estructura.

Aceleración

Máximo valor. $A_{v}^{i \text { deal }}=1,444 \mathrm{~m} / \mathrm{s}^{2}$

Punto: 91 (centro del vano 25).

Tren: A9.

Velocidad: $408 \mathrm{Km} / \mathrm{h}$.

$A_{v}=A_{v} \cdot\left(1+0,5 \varphi^{\prime \prime}\right)=1,444 m / s^{2}$

Valor límite: $0.35 \mathrm{~g}=3.5 \mathrm{~m} / \mathrm{s}^{2}$.

Conclusión: No se supera el límite de aceleración máxima permitido por la IAPF-07. 


\subsubsection{Curvas envolventes de aceleraciones máximas}

Envolventes de aceleración en puntos del vano 12 (sobre el arco)

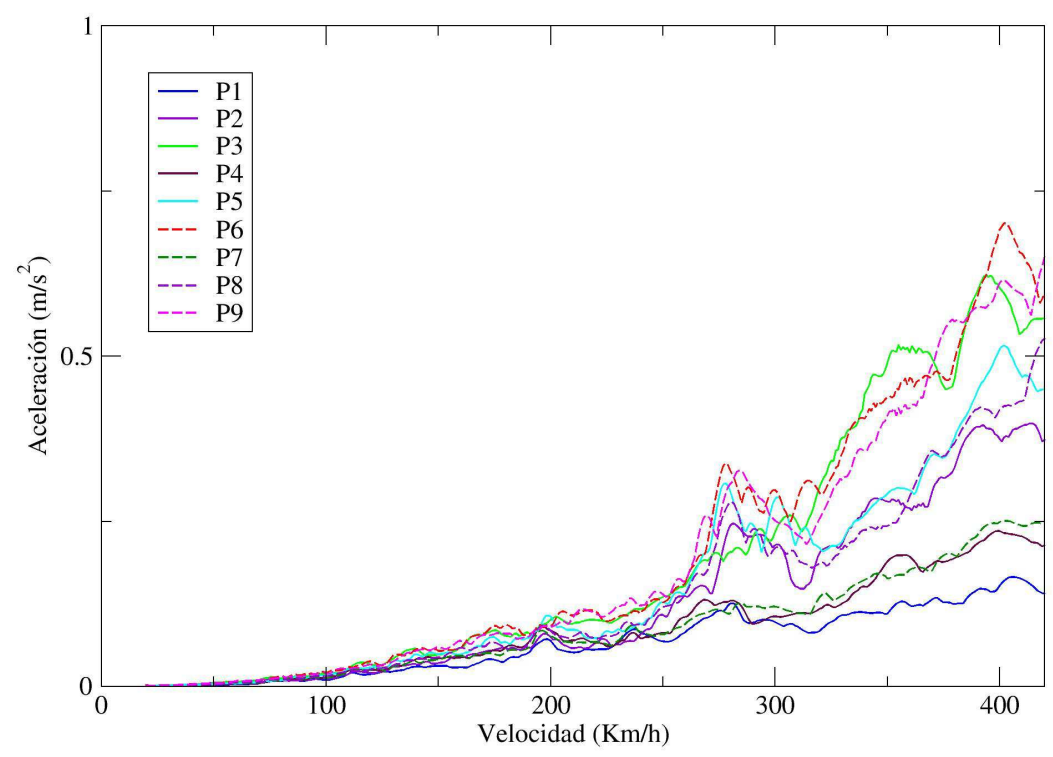

Figura 4.22: Envolventes de aceleración en puntos del vano 12 (sobre el arco).

Envolventes de aceleración en puntos del vano 13 (sobre el arco)

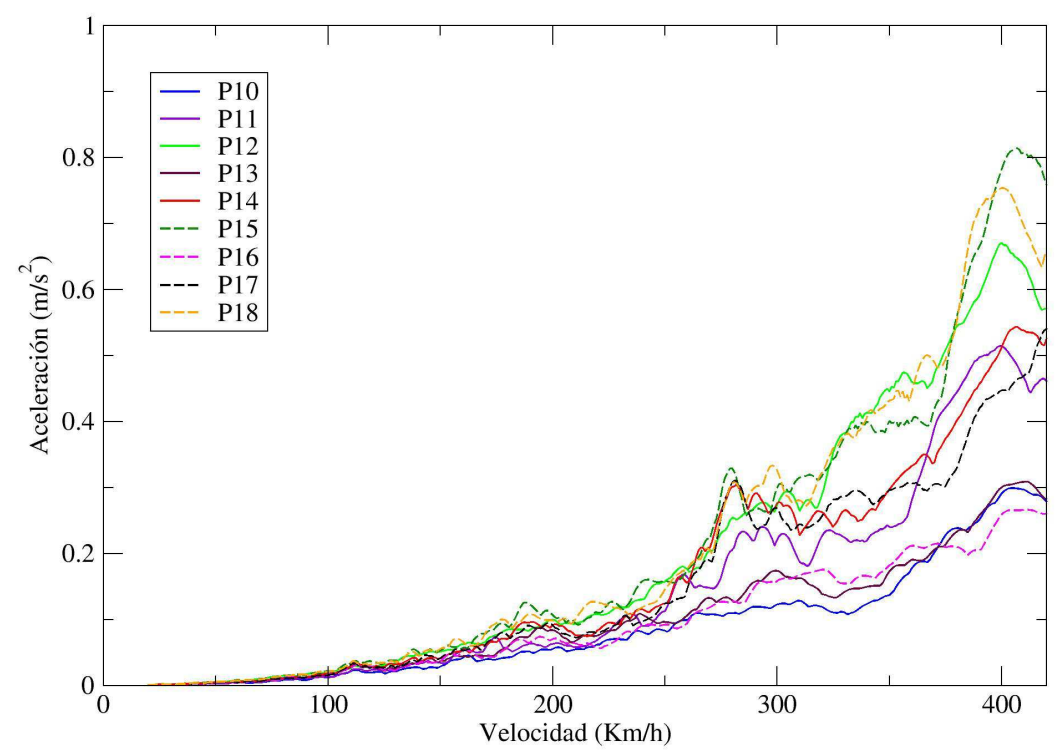

Figura 4.23: Envolventes de aceleración en puntos del vano 13 (sobre el arco). 
Envolventes de aceleración en puntos del vano 14 (sobre el arco)

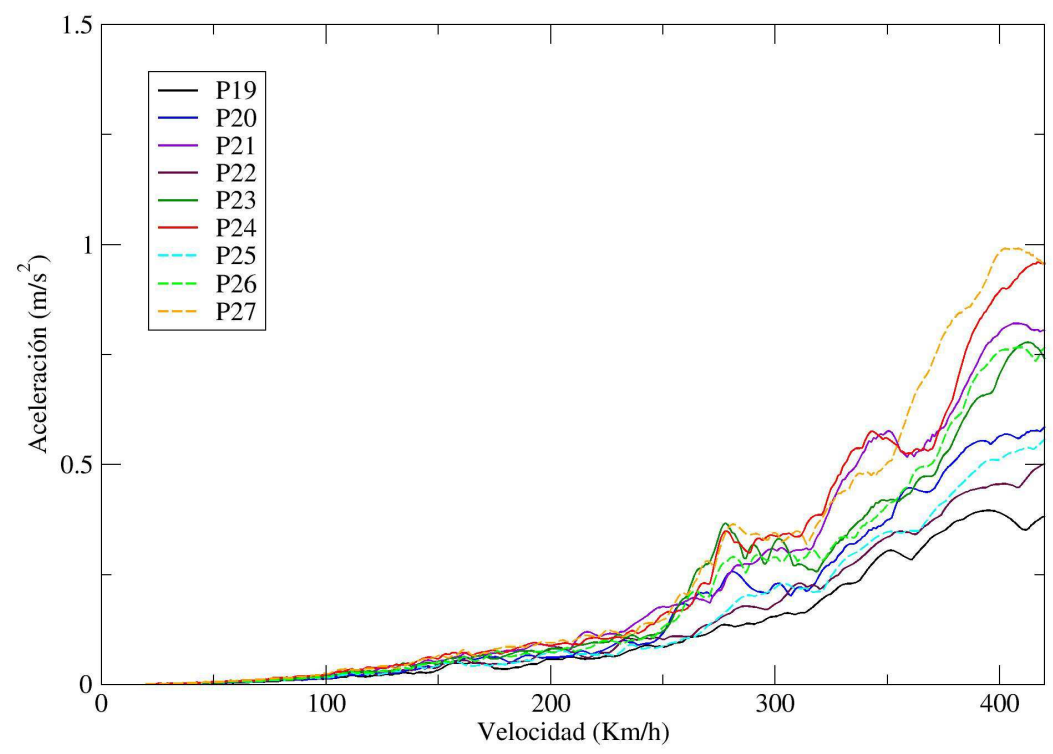

Figura 4.24: Envolventes de aceleración en puntos del vano 14 (sobre el arco).

Envolventes de aceleración en puntos del vano 15 (sobre el arco)

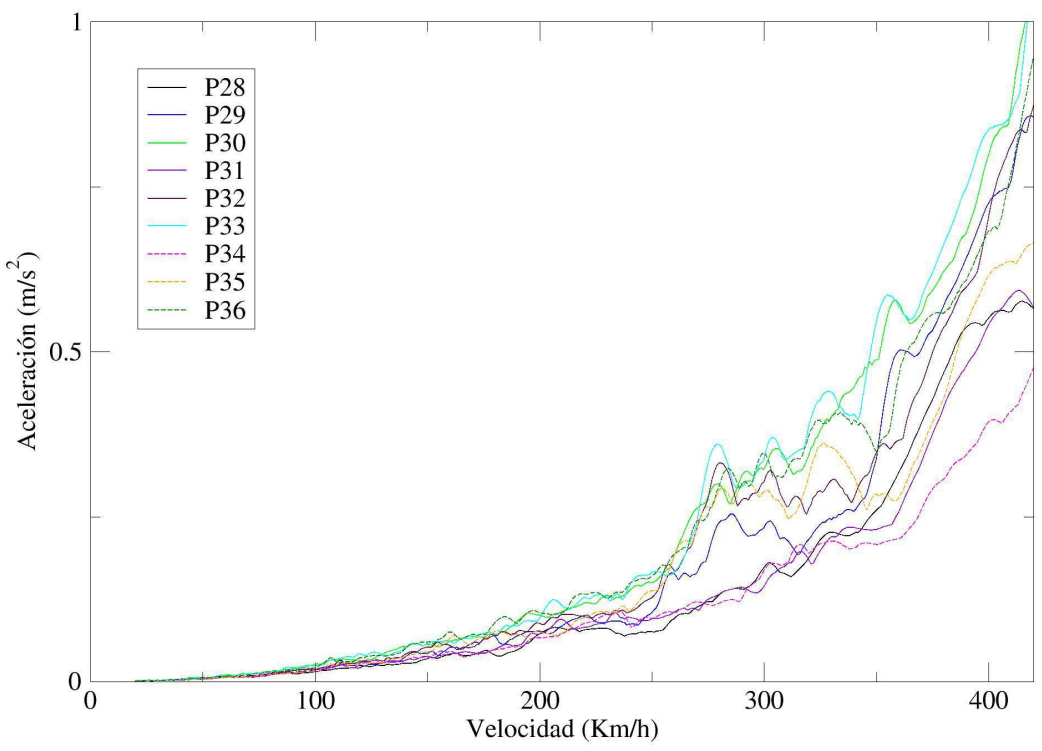

Figura 4.25: Envolventes de aceleración en puntos del vano 15 (sobre el arco). 
Envolventes de aceleración en puntos del vano 16 (sobre el arco)

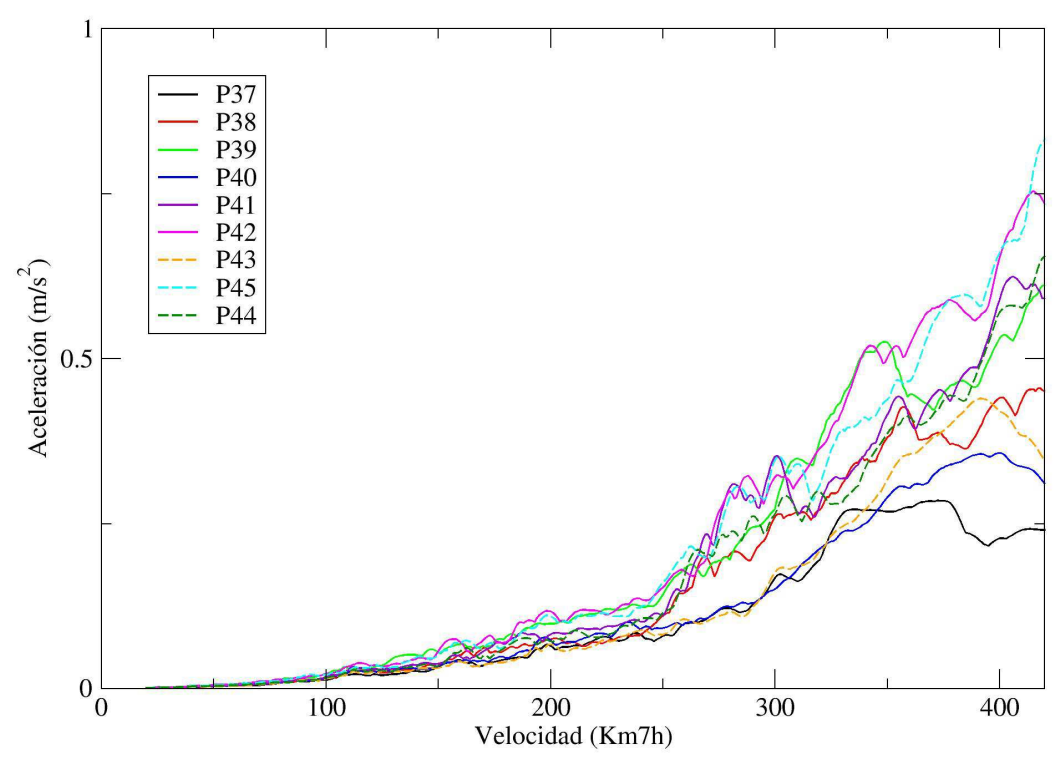

Figura 4.26: Envolventes de aceleración en puntos del vano 16 (sobre el arco).

Envolventes de aceleración en puntos del vano 17 (sobre el arco)

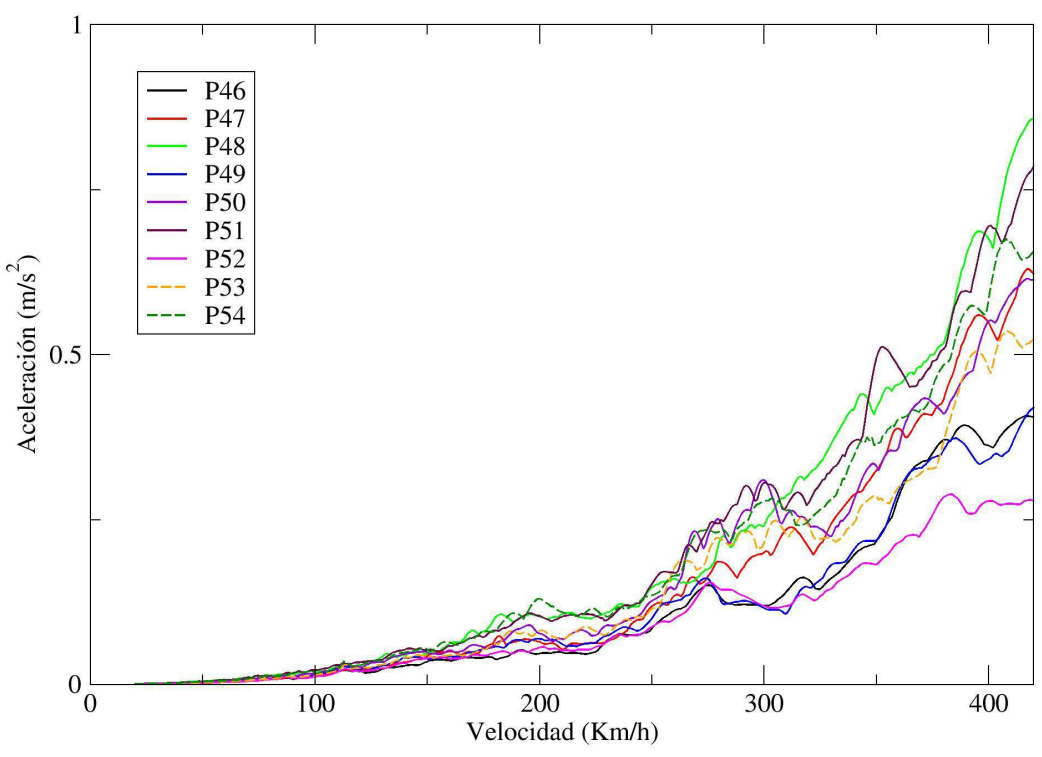

Figura 4.27: Envolventes de aceleración en puntos del vano 17 (sobre el arco). 
Envolventes de aceleración en puntos exteriores al arco (vanos 1-10)

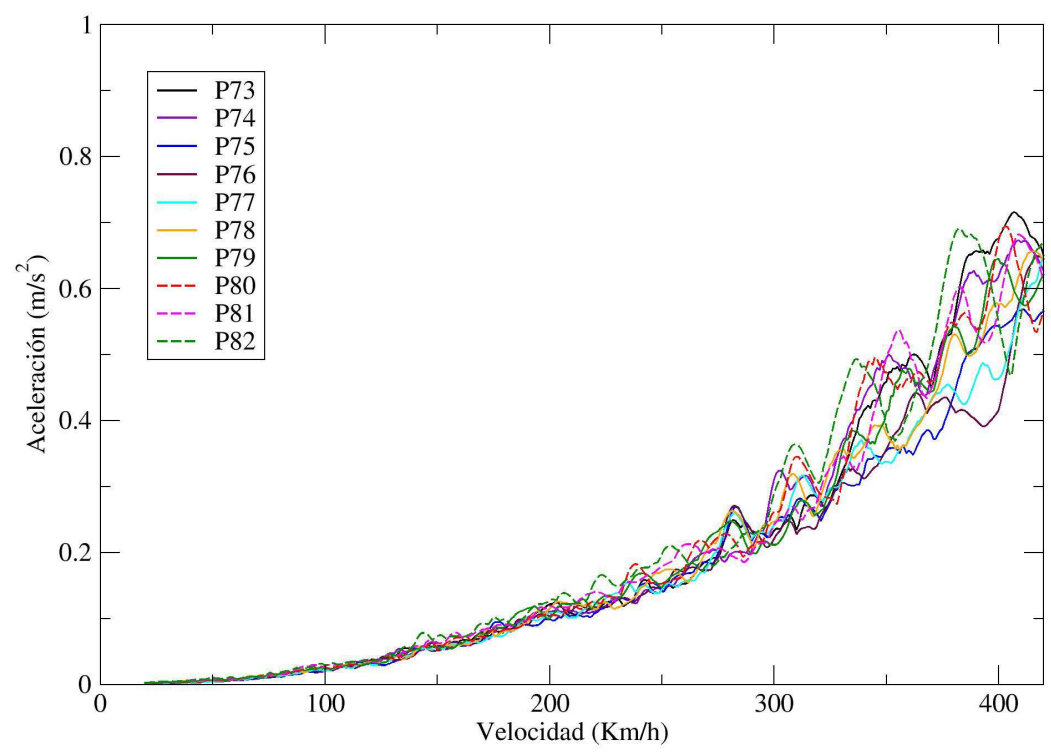

Figura 4.28: Envolventes de aceleración en puntos exteriores al arco (vanos 1-10).

Envolventes de aceleración en puntos exteriores al arco (vanos 11-,18-26)

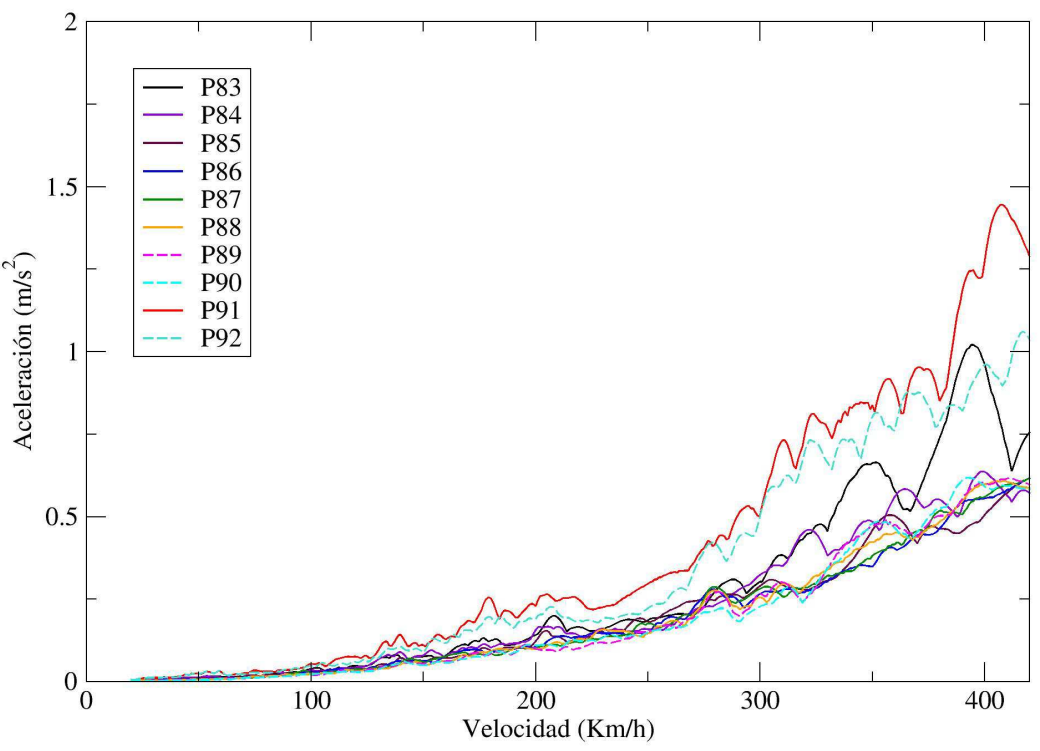

Figura 4.29: Envolventes de aceleración en puntos exteriores al arco (vanos 11,18-26). 
Envolventes de aceleración en el punto 33

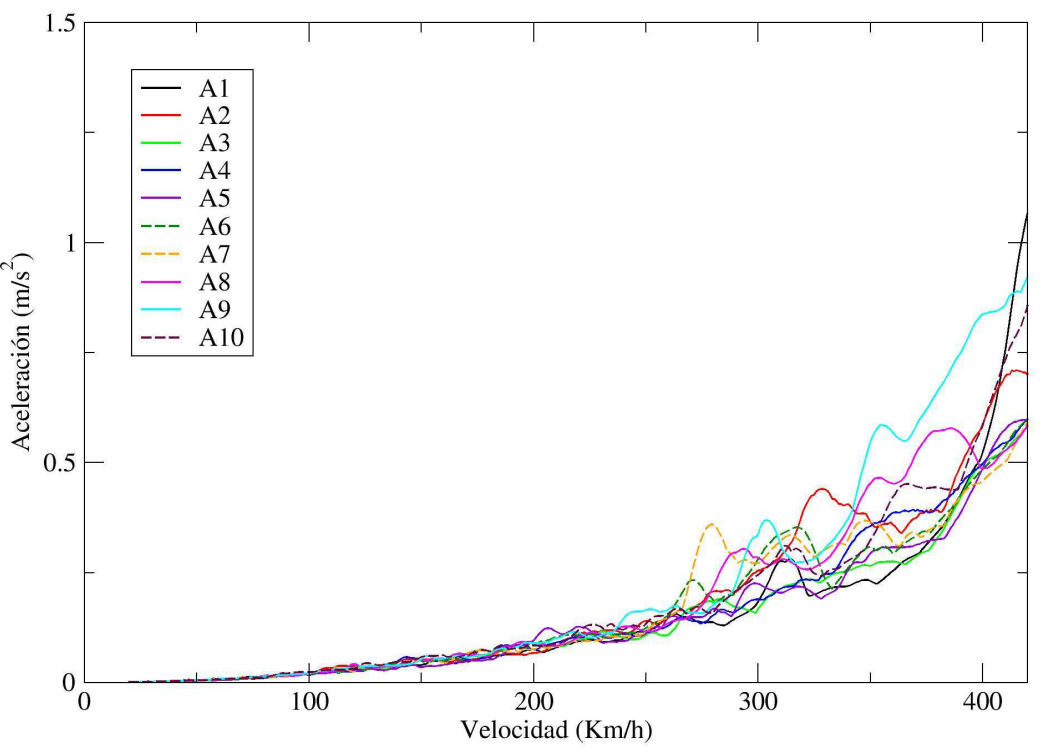

Figura 4.30: Envolventes de aceleración en el punto 33 (vano 15)

Envolventes de aceleración en el punto 91

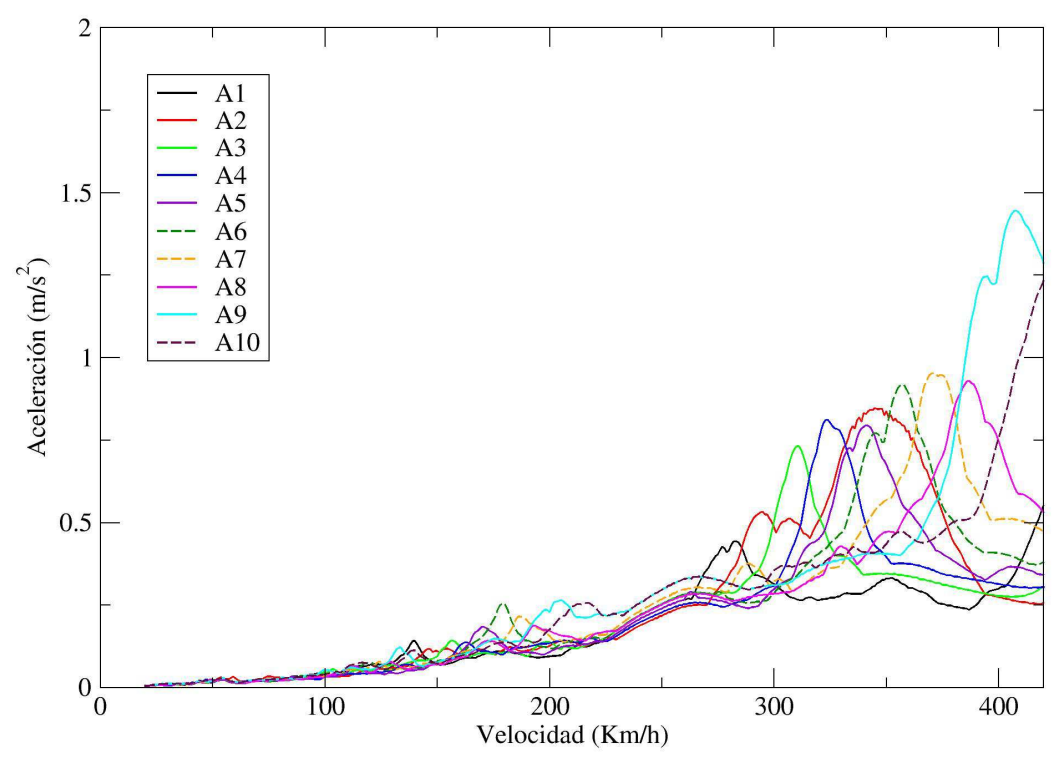

Figura 4.31: Envolventes de aceleración en el punto 91 (centro del vano 25) 


\subsubsection{Curvas envolventes de desplazamientos máximos}

Envolventes de desplazamiento en puntos del vano 12 (sobre el arco)

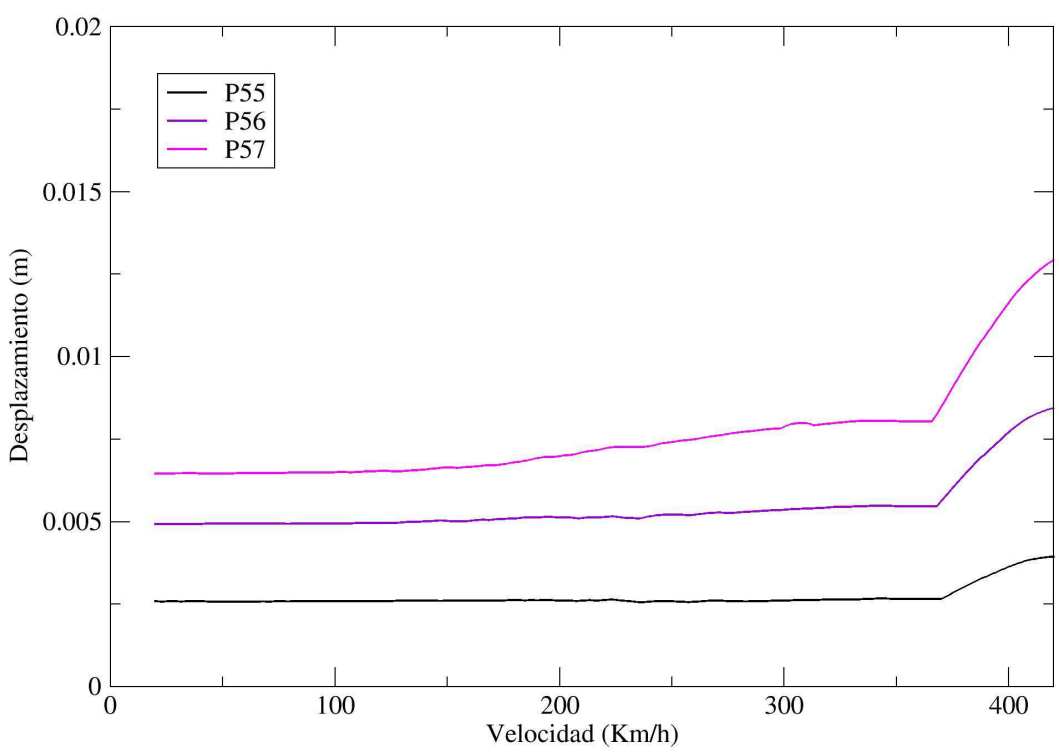

Figura 4.32: Envolventes de desplazamiento en puntos del vano 12 (sobre el arco).

Envolventes de desplazamiento en puntos del vano 13 (sobre el arco)

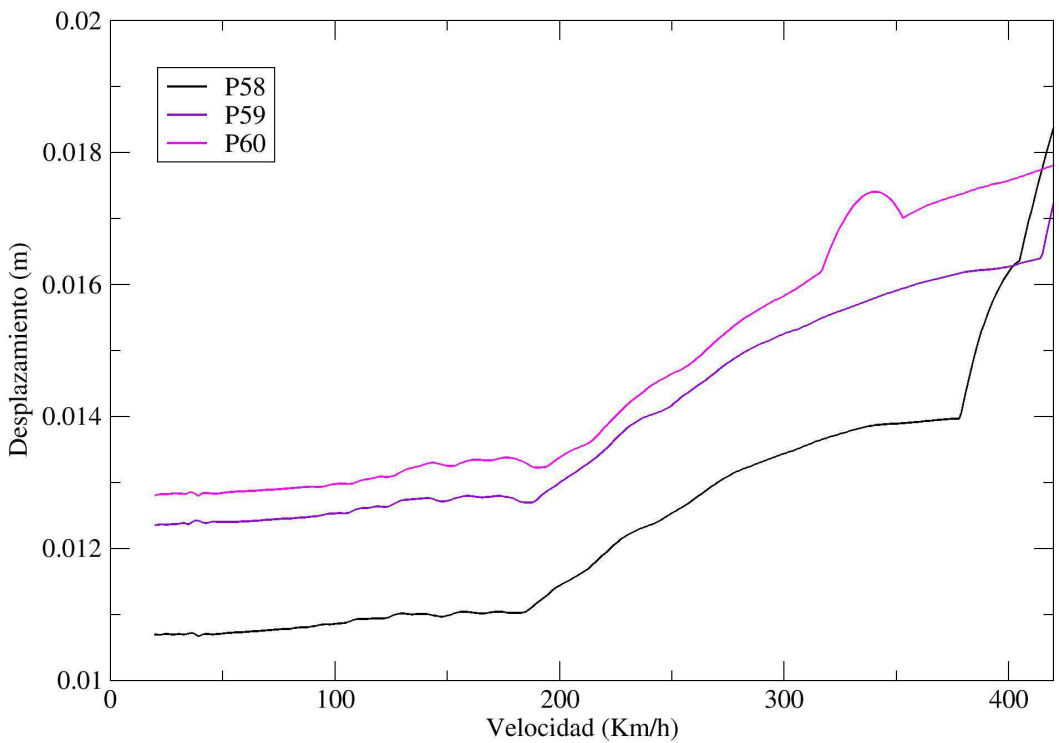

Figura 4.33: Envolventes de desplazamiento en puntos del vano 13 (sobre el arco). 
Envolventes de desplazamiento en puntos del vano 14 (sobre el arco)

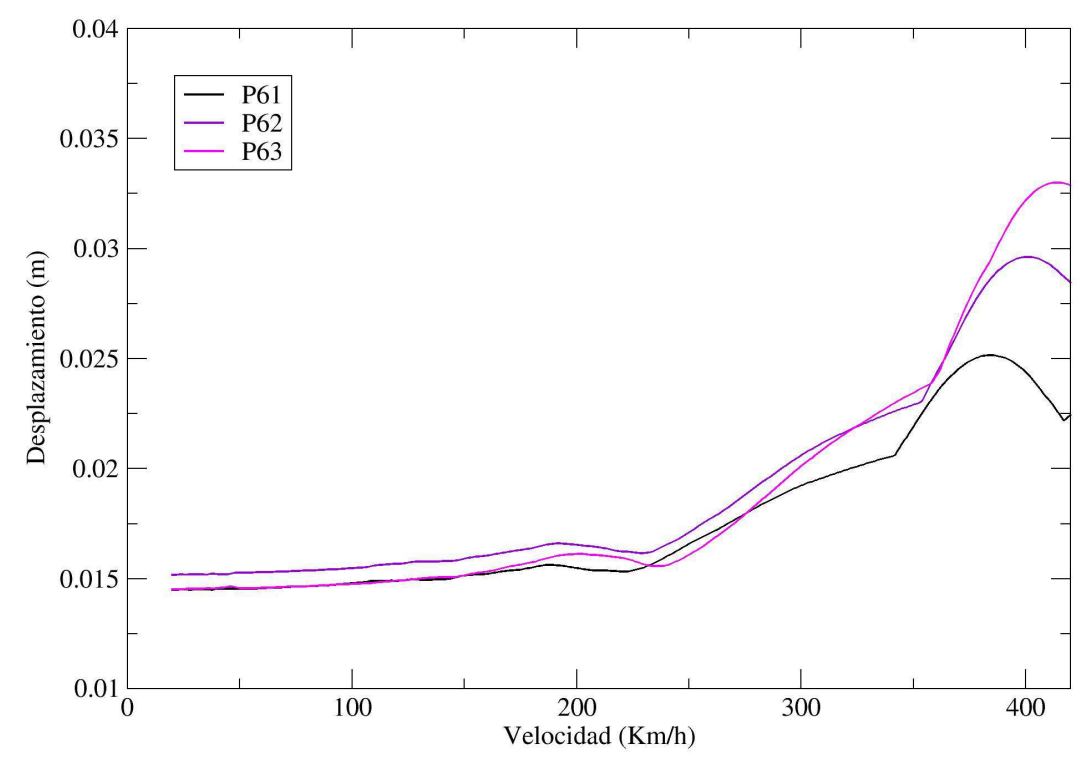

Figura 4.34: Envolventes de desplazamiento en puntos del vano 14 (sobre el arco).

Envolventes de desplazamiento en puntos del vano 15 (sobre el arco)

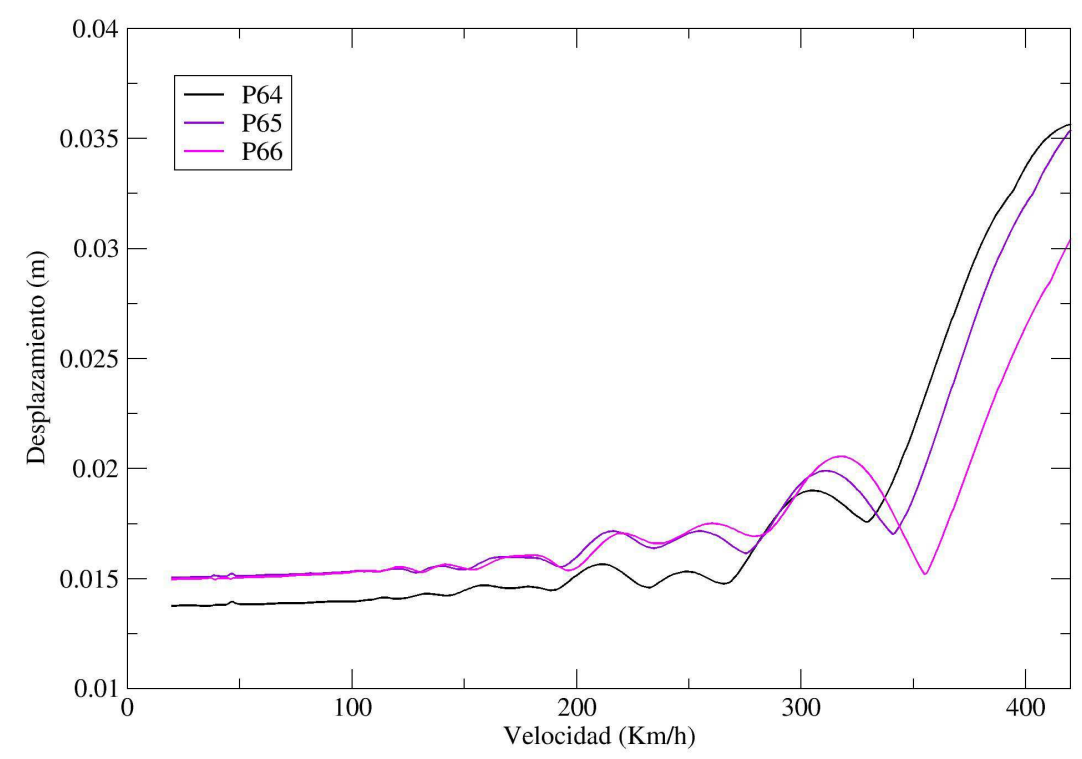

Figura 4.35: Envolventes de desplazamiento en puntos del vano 15 (sobre el arco). 
Envolventes de desplazamiento en puntos del vano 16 (sobre el arco)

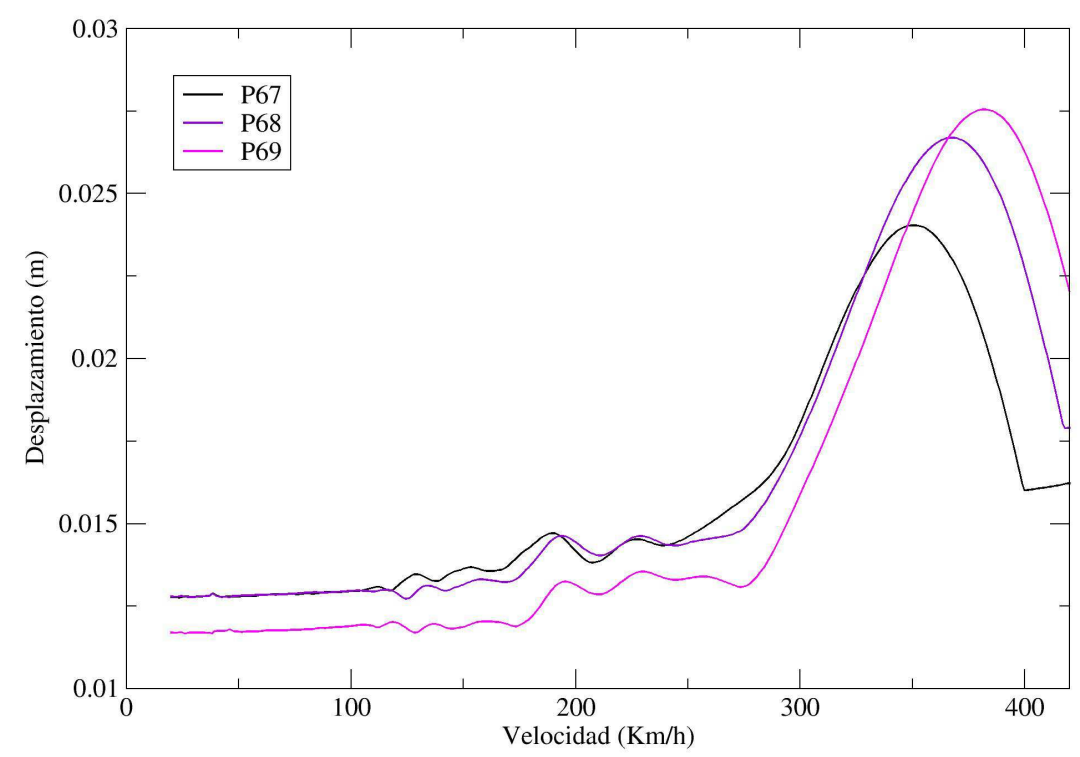

Figura 4.36: Envolventes de desplazamiento en puntos del vano 16 (sobre el arco).

Envolventes de desplazamiento en puntos del vano 17 (sobre el arco)

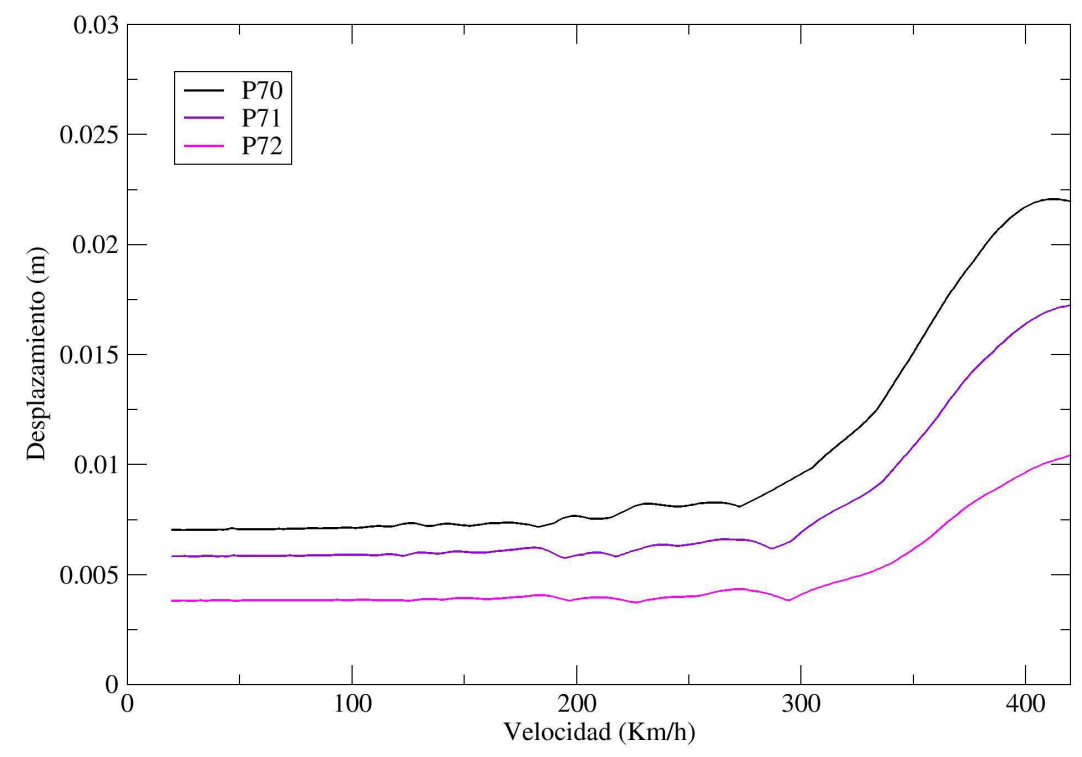

Figura 4.37: Envolventes de desplazamiento en puntos del vano 17 (sobre el arco). 
Envolventes de desplazamiento en el punto 64

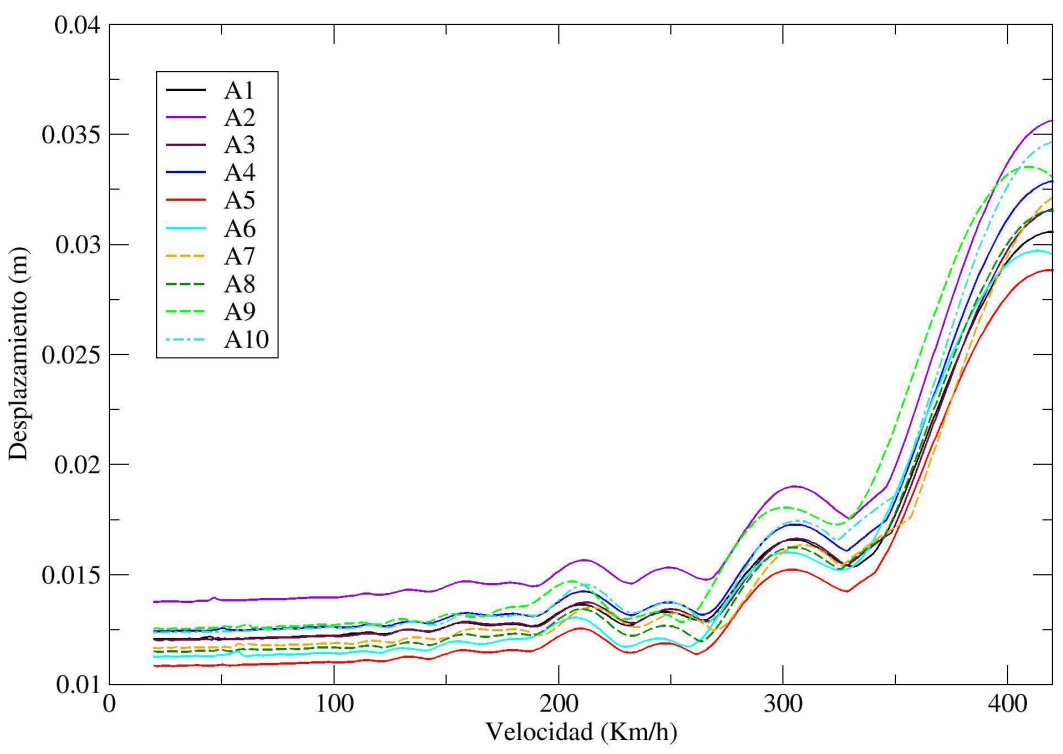

Figura 4.38: Envolventes de desplazamiento en el punto 64 (primer cuarto del vano 15, sobre el arco) 



\section{CAPÍtulo 5}

\section{Conclusiones}

A modo de resumen, se recogen en los siguientes puntos las conclusiones alcanzadas en todo el desarrollo de este Trabajo Fin de Máster:

1. El método semianalítico de resolución del problema de paso de cargas móviles presenta demostradas ventajas, en términos de precisión y tiempo de cálculo, frente a los métodos de integración incremental paso a paso. Esta metodología permite afrontar, de una manera realista, los pasos de carga de $\mathrm{Km} / \mathrm{h}$ en $\mathrm{Km} / \mathrm{h}$ aconsejados por el Eurocódigo, pasos necesarios para determinar con precisión la respuesta dinámica, y que de seguir las indicaciones de la instrucción IAPF, de paso de cargas de $10 \mathrm{~km} / \mathrm{h}$ en $10 \mathrm{Km} / \mathrm{h}$, no podrían ser registrados con exactitud.

2. Como ha quedado evidenciado según los resultados obtenidos, la estructura es válida. El arco sobre el río Tajo cumple la instrucción IAPF de forma holgada.

3. El análisis pone en evidencia la necesidad de realizar modelos 3D, debido a que existe torsión mixta que no es fácil tener en cuenta con modelos simplificados tipo viga o viga-torsión. La máxima aceleración se está dando en en un punto situado en los extremos de la cama de balasto, donde torsión y flexión son relevantes.

4. Necesidad de modelos que combinen la dinámica propia del arco con la del tablero, ya que, como se observa en el modo fundamental, son modos de periodos altos donde tablero y arco responden de forma acoplada.

5. Aunque las curvas de envolventes muestran los máximos a velocidades próximas al máximo teórico, exist resonancia, ya que observando las curvas, se producen los máximos siguiendo un patrón resonante. 



\section{Bibliografía}

[1] Instrucción de Acciones a considerar en el proyecto de Puentes de Ferrocarril (IAPF), 2007.

[2] P. Museros, A. Martínez-Castro, and A. Castillo-Linares. Semi-analytic solution in the time domain for non-uniform multi-span bernouilli-euler beams traversed by moving loads. Journal of Sounds and Vibration, 294:278-297,2006.

[3] UNE-EN 1991-2:2003. Eurocódigo 1: Acciones en estructuras. Parte 2: Cargas de tráfico en puentes. Septiembre 2003.

[4] Fiche 776-1R. «Charges a prender en consideration dans le calcul des ponts-rails». 5 Ed, Agosto de 2006.

[5] Comité D214 sobre puentes de ferrocarril para velocidades superiores a $200 \mathrm{Km} / \mathrm{h}$. ERRI D214 (a. Design of Railway Bridges for Speed up to $350 \mathrm{Km} / \mathrm{h}$; Dynamic loadings effects including resonance. Technical report, european Rail Research institude.,1998.

[6] J. Dominguez-Barbero. Dinámica de puentes de ferrocarril para alta velocidad: métods de cálculo y estudio de la resonancia. PhD thesis, Univeridad Politécnica de Madrid., 2001.

[7] K. Liu, E. Reynders, G. De Roeck, and G.Lombaert. Experimental and numerical analysis of a composite bridge for high-speed trains. Journal of Sounds and Vibration, 320:201-220, 2009.

[8] L. Frýba. Dinamic behaviour of bridges due to high-speed trains, in: R. Delgado, R. Calçada, A. Campos (eds.). In Workshop Bridges for High-Speed railways, pages 137-158, Faculty of Enegineering, University os Oporto, 2004.

[9] F. Mancel. Cedypia: Analytical software for calculating dynamic effects on railway bridges. In Proceedings of the Fourth European Conference on Structural Dynamics (Eurodyn'99), volume 2, pages 669-674. A.A. Balkema, 1999.

[10] F. Bleich. Theorie und Berechnung der Eisernen Brücken. Universidad de Michigan, 1924.

[11] A. Kryloff. Über die erzwungenen schwingungen von gleichförmingen elastichen stäben. Mathematische Annalen, 1995.

[12] L. Frýba. Vibration of solids and structures under moving loads. 3rd ed. Thomas Telford, 1999.

[13] J. Li and M. Su. The resonant vibration for a simply supported girder bridge under high-speed trains. Journal of Sounds and Vibration, 224:897-915. DOI:10.1006/jsvi.1999.2226, 1999.

[14] Y.B. Yang, J.D. Yau, and L.C. Hsu. Vibration of simple beams due to trains moving at high speeds. Engineering Structures, 19(11).936-944. DOI:10.1016/S0141-0296(97)00001-1,1997.

[15] Y.H. Chen and C.Y. Li. Dynamic response of elevated high-speed railway. Journal of Bridge Enegineering, 5:124-130,2000.

[16] T. Hayashikawa and N. Watanabe. Dynamic behaviour of continuous beams with moving loads.Journal of the Engineering Mechanics Division,ASCE,107:229-246,1981.

[17] K. Henchi, M. Fafard, G. Dhatt, and M. Talbot. Dynamic behaviour of multispan beams under moving loads. Journal of Sounds and Vibration,199(1):33-50. doi:10.1006/jsvi.1996.0628,1997.

[18] R.W.Clough and J. Penzien. Dynamics of Structures. Mac Graw-Hill., 1993.

[19] N.M.Newmark. A method of computation for structural dynamics. ASCE, 85, 1959. 